\title{
RENORMALIZATION OF POINCARE TRANSFORMATIONS IN HAMILTONIAN SEMICLASSICAL FIELD THEORY
}

\author{
O.Yu. Shvedov \\ Sub-Dept. of Quantum Statistics and Field Theory, \\ Department of Physics, Moscow State University \\ Vorobievy gory, Moscow 119899, Russia
}

October 26, 2018

hep-th/0109142

\begin{abstract}
Semiclassical Hamiltonian field theory is investigated from the axiomatic point of view. A notion of a semiclassical state is introduced. An "elementary" semiclassical state is specified by a set of classical field configuration and quantum state in this external field. "Composed" semiclassical states viewed as formal superpositions of "elementary" states are nontrivial only if the Maslov isotropic condition is satisfied; the inner product of "composed" semiclassical states is degenerate. The mathematical proof of Poincare invariance of semiclassical field theory is obtained for "elementary" and "composed" semiclassical states. The notion of semiclassical field is introduced; its Poincare invariance is also mathematically proved.
\end{abstract}

PACS numbers: 11.30.Cp, 11.10.Cd, 11.15.Kc, 11.10.Gh.

\footnotetext{
${ }^{0}$ e-mail: shvedov@qs.phys.msu.su
} 


\section{Introduction}

Different approaches to semiclassical field theory have been developed. Most of them were based on the functional integral technique: physical quantities were expressed via functional integrals which were evaluated with the help of saddle-point or stationary-phase technique. Since energy spectrum and $S$ matrix elements can be found from the functional integral [1, 2], this approach appeared to be useful for the soliton quantization theory [1, 2, 3, 4, 5].

Another important partial case of the semiclassical field theory is the theory of quantization in a strong external background classical field [6] or in curved space-time [7]: one decomposes the field as a sum of a classical c-number component and a quantum component. Then the theory is quantized.

The one-loop approximation [8, 9, 10, 11], the time-dependent Hartree-Fock approximation [8, 9, 12, 13] and the Gaussian approximation developed in [14, 15, 16, 17] may be also viewed as examples of applications of semiclassical conceptions.

On the other hand, the axiomatic field theory [18, 19, 20] tells us that main objects of QFT are states and observables. The Poincare group is represented in the Hilbert state space, so that evolution, boosts and other Poincare transformations are viewed as unitary operators.

The purpose of this paper is to introduce the semiclassical analogs of such QFT notions as states, fields and Poincare transformations. The analogs of Wightman Poincare invariance and field axioms for the semiclassical field theory are to be formulated and checked.

Unfortunately, "exact" QFT is mathematically constructed for a restricted class of models only (see, for example, [21, 22, 23, 24]). Therefore, formal approximate methods such as perturbation theory seem to be ways to quantize the field theory rather than to construct approximations for the exact solutions of QFT equations. The conception of field quantization within the perturbation framework is popular [25, 26]. One can expect that the semiclassical approximation plays an analogous role.

To construct the semiclassical formalism based on the notion of a state, one should use the equationof-motion formulation of QFT rather than the usual $S$-matrix formulation. It is well-known that additional difficulties such as Stueckelberg divergences [27 and problems associated with the Haag theorem [28, 19, 20] arise in the equation-of-motion approach. There are some ways to overcome them. The vacuum divergences can be eliminated in the perturbation theory with the help of the Faddeev transformation [29]. Stueckelberg divergences can be treated analogously [30] (exactly solvable models with Stueckelberg divergences have been suggested recently [31, 32]). These investigations are important for the semiclassical Hamiltonian field theory [33].

The semiclassical approaches are formally applicable to the quantum field theory models if the Lagrangian depends on the fields $\varphi$ and the small parameter $\lambda$ as follows (see, for example, 幽):

$$
\mathcal{L}=\frac{1}{2} \partial_{\mu} \varphi \partial_{\mu} \varphi-\frac{m^{2}}{2} \varphi^{2}-\frac{1}{\lambda} V(\sqrt{\lambda} \varphi)
$$

where $V$ is an interaction potential. To illustrate the formal semiclassical ansatz for the state vector, use the functional Schrodinger representation (see, for example, [12, 13, 16, 17]). States at fixed moment of time are represented as functionals $\psi[\varphi(\cdot)]$ depending on fields $\varphi(\mathbf{x}), \mathbf{x} \in \mathbf{R}^{d}$, the field operator $\hat{\varphi}(\mathbf{x})$ is the operator of multiplication by $\varphi(\mathbf{x})$, while the canonically conjugated momentum $\hat{\pi}(\mathbf{x})$ is represented as a differentiation operator $-i \delta / \delta \varphi(\mathbf{x})$. The functional Schrodinger equation reads

$$
i \frac{d \psi^{t}}{d t}=\mathcal{H} \psi^{t}
$$

where

$$
\mathcal{H}=\int d \mathbf{x}\left[-\frac{1}{2} \frac{\delta^{2}}{\delta \varphi(\mathbf{x}) \delta \varphi(\mathbf{x})}+\frac{1}{2}(\nabla \varphi)^{2}(\mathbf{x})+\frac{m^{2}}{2} \varphi^{2}(\mathbf{x})+\frac{1}{\lambda} V(\sqrt{\lambda} \varphi(\mathbf{x}))\right]
$$

The simplest semiclassical state corresponds to the Maslov theory of complex germ in a point [34, 35, 36]. It depends on the small parameter $\lambda$ as

$$
\psi^{t}[\varphi(\cdot)]=e^{\frac{i}{\lambda} S^{t}} e^{\frac{i}{\lambda} \int d \mathbf{x} \Pi^{t}(\mathbf{x})\left[\varphi(\mathbf{x}) \sqrt{\lambda}-\Phi^{t}(\mathbf{x})\right]} f^{t}\left(\varphi(\cdot)-\frac{\Phi^{t}(\cdot)}{\sqrt{\lambda}}\right) \equiv\left(K_{S^{t}, \Pi^{t}, \Phi^{t}} f^{t}\right)[\varphi(\cdot)],
$$


where $S^{t}, \Pi^{t}(\mathbf{x}), \Phi^{t}(\mathbf{x}), t \in \mathbf{R}, \mathbf{x} \in \mathbf{R}^{d}$ are smooth real functions which rapidly damp with all their derivatives as $\mathbf{x} \rightarrow \infty, f^{t}[\phi(\cdot)]$ is a $t$-dependent functional.

As $\lambda \rightarrow 0$, the substitution (1.3) satisfies eq.(1.2) in the leading order in $\lambda$ if the following relations are obeyed. First, for the "action" $S^{t}$ one finds,

$$
\frac{d S^{t}}{d t}=\int d \mathbf{x}\left[\Pi^{t}(\mathbf{x}) \dot{\Phi}^{t}(\mathbf{x})-\frac{1}{2}\left(\Pi^{t}(\mathbf{x})\right)^{2}-\frac{1}{2}\left(\nabla \Phi^{t}(\mathbf{x})\right)^{2}-\frac{m^{2}}{2}\left(\Phi^{t}(\mathbf{x})\right)^{2}-V\left(\Phi^{t}(\mathbf{x})\right)\right],
$$

Second, $\Pi^{t}, \Phi^{t}$ obeys the classical Hamiltonian system

$$
\dot{\Phi}^{t}=\Pi^{t},-\dot{\Pi}^{t}=\left(-\Delta+m^{2}\right) \Phi^{t}+V^{\prime}\left(\Phi^{t}\right),
$$

Finally, the functional $f^{t}$ satisfies the functional Schrodinger equation with the quadratic Hamiltonian

$$
i \dot{f}^{t}[\phi(\cdot)]=\int d \mathbf{x}\left[-\frac{1}{2} \frac{\delta^{2}}{\delta \phi(\mathbf{x}) \delta \phi(\mathbf{x})}+\frac{1}{2}(\nabla \phi(\mathbf{x}))^{2}+\frac{m^{2}}{2} \phi^{2}(\mathbf{x})+\frac{1}{2} V^{\prime \prime}\left(\Phi^{t}(\mathbf{x})\right) \phi^{2}(\mathbf{x})\right] f^{t}[\phi(\cdot)] .
$$

There are more complicated semiclassical states that also approximately satisfy the functional Schrodinger equation (1.2). These ansatzes correspond to the Maslov theory of Lagrangian manifolds with complex germs [34, 35, 36]. They are discussed in section 5 .

However, the QFT divergences lead to the following difficulties.

It is not evident how one should specify the class of possible functionals $f$ and introduce the inner product on such a space via functional integral. This class was constructed in [33]. In particular, it was found when the Gaussian functional

$$
f[\phi(\cdot)]=\text { const } \exp \left(\frac{i}{2} \int d \mathbf{x} d \mathbf{y} \phi(\mathbf{x}) \phi(\mathbf{y}) \mathcal{R}(\mathbf{x}, \mathbf{y})\right)
$$

belongs to this class. The condition on the quadratic form $\mathcal{R}$ which was obtained in [33] depends on $\Phi$, $\Pi$ and differs from the analogous condition in the free theory. This is in agreement with the statement of [37, 38] that nonequivalent representations of the canonical commutation relations at different moments of time should be considered if QFT in the strong external field is investigated in the leading order in $\lambda$. However, this does not lead to non-unitarity of the exact theory: the simple example has been presented in [32].

Another problem is to formulate the semiclassical theory in terms of the axiomatic field theory. Section 2 deals with formulation of axioms of relativistic invariance and field for the semiclassical theory. Section 3 is devoted to construction of Poincare transformations. In section 4 the notion of semiclassical field is investigated. More complicated semiclassical states are constructed in section 5 . Section 6 contains concluding remarks.

\section{Axioms of semiclassical field theory}

In the Wightman axiomatic approach the main object of QFT is a notion of a state space [18, 19, 20]. Formula (1.3) shows us that in the semiclassical field theory a state at fixed moment of time should be viewed as a set $(S, \Pi(\cdot), \Phi(\cdot), f[\phi(\cdot)])$ of a real number $S$, real functions $\Pi(\mathbf{x}), \Phi(\mathbf{x}), \mathbf{x} \in \mathbf{R}^{d}$ and a functional $f[\phi(\cdot)]$ from some class. This class depends on $\Pi$ and $\Phi$. Superposition of semiclassical states $\left(S_{1}, \Pi_{1}, \Phi_{1}, f_{1}\right)$ and $\left(S_{2}, \Pi_{2}, \Phi_{2}, f_{2}\right)$ is of the semiclassical type (1.3) if and only if $S_{1}=S_{2}, \Phi_{1}=\Phi_{2}$, $\Pi_{1}=\Pi_{2}$.

Thus, one introduces [39, 40] the structure of a vector bundle (called as a "semiclassical bundle" in [40]) on the set of semiclassical states of the type (11.3). The base of the bundle being a space of sets $(S, \Pi, \Phi)$ ("extended phase space" [39]) will be denoted as $\mathcal{X}$. The fibers are classes of functionals which depend on $\Phi$ and $\Pi$. Making use of the result concerning the class of functionals [33], one makes 
the bundle trivial as follows. Consider the $\Phi, \Pi$ - dependent mapping $V$ which defines a correspondence between functionals $f$ and elements of the Fock space $\mathcal{F}$ :

$$
V: \Psi \mapsto f, \quad \Psi \in \mathcal{F}, \quad f=f[\phi(\cdot)] .
$$

as follows (see, for example, 41]). Let $\tilde{\mathcal{R}}(\mathbf{x}, \mathbf{y})$ be an $\Phi, \Pi$ - dependent symmetric function such that its imaginary part is a kernel of a positively definite operator and the condition of ref. [33] (see eq.(3.41) of subsection 3.6) is satisfied. . By $\hat{\mathcal{R}}$ we denote the operator with kernel $\tilde{\mathcal{R}}$, while $\hat{\Gamma}$ has a kernel $i^{-1}\left(\tilde{\mathcal{R}}-\tilde{\mathcal{R}}^{*}\right)$. The vacuum vector of the Fock space corresponds to the Gaussian functional (1.7)). The operator $V$ is uniquely defined from the relations

$$
\begin{gathered}
V^{-1} \phi(\mathbf{x}) V=i\left(\hat{\Gamma}^{-1 / 2}\left(A^{+}-A^{-}\right)\right)(\mathbf{x}), \\
V^{-1} \frac{1}{i} \frac{\delta}{\delta \phi(\mathbf{x})} V=i\left(\hat{\mathcal{R}} \hat{\Gamma}^{-1 / 2} A^{+}-\hat{\mathcal{R}}^{*} \hat{\Gamma}^{-1 / 2} A^{-}\right)(\mathbf{x}) .
\end{gathered}
$$

Here $A^{ \pm}(\mathbf{x})$ are creation and annihilation operators in the Fock space.

Definition 2.1. A semiclassical state is a point on the trivial bundle $\mathcal{X} \times \mathcal{F} \rightarrow \mathcal{X}$.

An important postulate of QFT is Poincare invariance. This means that a representation of the Poincare group in the state space should be specified. For each Poincare transformation of the form

$$
x^{\prime \mu}=\Lambda_{\nu}^{\mu} x^{\nu}+a^{\mu}, \quad \mu, \nu=\overline{0, d}
$$

which is denoted as $(a, \Lambda)$, the unitary operator $\mathcal{U}_{a, \Lambda}$ should be specified. The group property

$$
\mathcal{U}_{\left(a_{1}, \Lambda_{1}\right)} \mathcal{U}_{\left(a_{2}, \Lambda_{2}\right)}=\mathcal{U}_{\left(a_{1}, \Lambda_{1}\right)\left(a_{2}, \Lambda_{2}\right)}
$$

with

$$
\left(a_{1}, \Lambda_{1}\right)\left(a_{2}, \Lambda_{2}\right)=\left(a_{1}+\Lambda_{1} a_{2}, \Lambda_{1} \Lambda_{2}\right)
$$

should be satisfied.

Formulate an analog of the Poincare invariance axiom for the semiclassical theory. Suppose that the Poincare transformation $\mathcal{U}_{a, \Lambda}$ takes any semiclassical state $(X, f)$ to a semiclassical state $(\tilde{X}, \tilde{f})$ in the leading order in $\lambda^{1 / 2}$. Denote $\tilde{X}=u_{a, \Lambda} X, \tilde{f}=U\left(u_{a, \Lambda} X \leftarrow X\right) f$.

\section{Axiom 1 (Poincare invariance)}

() the mappings $u_{a, \Lambda}: \mathcal{X} \rightarrow \mathcal{X}$ are specified, the group properties for them $u_{a_{1}, \Lambda_{1}} u_{a_{2}, \Lambda_{2}}=u_{\left(a_{1}, \Lambda_{1}\right)\left(a_{2}, \Lambda_{2}\right)}$ are satisfied;

() for all $X \in \mathcal{X}$ the unitary operators $U_{a, \Lambda}\left(u_{a, \Lambda} X \leftarrow X\right): \mathcal{F} \rightarrow \mathcal{F}$, obeying the group property

$$
\begin{gathered}
U_{a_{1}, \Lambda_{1}}\left(u_{\left(a_{1}, \Lambda_{1}\right)\left(a_{2}, \Lambda_{2}\right)} X \leftarrow u_{\left(a_{2}, \Lambda_{2}\right)} X\right) U_{a_{2}, \Lambda_{2}}\left(u_{\left(a_{2}, \Lambda_{2}\right)} X \leftarrow X\right)= \\
U_{\left(a_{1}, \Lambda_{1}\right)\left(a_{2}, \Lambda_{2}\right)}\left(u_{\left(a_{1}, \Lambda_{1}\right)\left(a_{2}, \Lambda_{2}\right)} X \leftarrow X\right)
\end{gathered}
$$

are specified.

An important feature of QFT is the notion of a field: it is assumed that an operator distribution $\hat{\varphi}(\mathbf{x}, t)$ is specified. Investigate it in the semiclassical theory. Applying the operator $\varphi(\mathbf{x})$ to the semiclassical state (1.3), we obtain an analogous state:

$$
e^{\frac{i}{\lambda} S^{t}} e^{\frac{i}{\lambda} \int d \mathbf{x} \Pi^{t}(\mathbf{x})\left[\varphi(\mathbf{x}) \sqrt{\lambda}-\Phi^{t}(\mathbf{x})\right]} \tilde{f}^{t}\left(\varphi(\cdot)-\frac{\Phi^{t}(\cdot)}{\sqrt{\lambda}}\right)
$$

where

$$
\tilde{f}^{t}[\phi(\cdot)]=\left(\lambda^{-1 / 2} \Phi^{t}(\mathbf{x})+\phi(\mathbf{x})\right) f^{t}[\phi(\cdot)]
$$

As $\lambda \rightarrow 0$, one has

$$
\hat{\varphi}(\mathbf{x}, t)=\lambda^{-1 / 2} \Phi^{t}(\mathbf{x})+\hat{\phi}(\mathbf{x}, t: X)
$$

where $\hat{\phi}(\mathbf{x}, t: X)$ is a $\Pi, \Phi$-dependent operator in $\mathcal{F}, \Phi^{t}(\mathbf{x}) \equiv \Phi(x: X)$ is a solution to the Cauchy problem for eq.(1.5). The field axiom can be reformulated as follows. 
Axiom 2. For each $X \in \mathcal{X}$ the operator distribution $\phi(\mathbf{x}, t ; X): \mathcal{F} \rightarrow \mathcal{F}$ is specified.

An important feature of the relativistic quantum field theory is the property of Poincare invariance of fields. The operator distribution $\hat{\varphi}(\mathbf{x}, t)$ should obey the following property

$$
\mathcal{U}_{a, \Lambda} \hat{\varphi}(x)=\hat{\varphi}(\Lambda x+a) \mathcal{U}_{a, \Lambda}
$$

Apply this identity to a semiclassical state $(X, f)$. In leading orders in $\lambda^{1 / 2}$, one obtains:

$$
\begin{gathered}
\lambda^{-1 / 2} \Phi(x: X)\left(u_{a, \Lambda} X, U_{a, \Lambda}\left(u_{a, \Lambda} X \leftarrow X\right) f\right)+\left(u_{a, \Lambda} X, U_{a, \Lambda}\left(u_{a, \Lambda} X \leftarrow X\right) \hat{\phi}(x: X) f\right)= \\
\lambda^{-1 / 2} \Phi\left(\Lambda x+a: u_{a, \Lambda} X\right)\left(u_{a, \Lambda} X, U_{a, \Lambda}\left(u_{a, \Lambda} X \leftarrow X\right) f\right) \\
+\left(u_{a, \Lambda} X, \hat{\phi}\left(\Lambda x+a: u_{a, \Lambda} X\right) U_{a, \Lambda}\left(u_{a, \Lambda} X \leftarrow X\right) f\right) .
\end{gathered}
$$

Therefore, we formulate the following axiom.

Axiom 3. (Poincare invariance of fields). The following properties are satisfied:

$$
\begin{gathered}
\Phi(x: X)=\Phi\left(\Lambda x+a: u_{a, \Lambda} X\right) \\
\hat{\phi}\left(\Lambda x+a: u_{a, \Lambda} X\right) U_{a, \Lambda}\left(u_{a, \Lambda} X \leftarrow X\right)=U_{a, \Lambda}\left(u_{a, \Lambda} X \leftarrow X\right) \hat{\phi}(x: X) .
\end{gathered}
$$

\section{Semiclassical Poincare transformations}

\subsection{Construction of poincare transformations in the functional represen- tation}

1. Let us construct the mappings $u_{a, \Lambda}$ and unitary opertaors $U_{a, \Lambda}\left(u_{a, \lambda} X \leftarrow X\right)$. Since any Poincare transformation is a composition of time and space translations, boost and spatial rotations,

$$
(a, \Lambda)=\left(a^{0}, 0\right)(\mathbf{a}, 0)\left(0, \exp \left(\alpha^{k} l^{0 k}\right)\right)\left(0, \exp \left(\frac{1}{2} \theta_{s m} l^{s m}\right)\right)
$$

with $\theta_{s m}=-\theta_{m s}$,

$$
\left(l^{\lambda \mu}\right)_{\beta}^{\alpha}=-g^{\lambda \alpha} \delta_{\beta}^{\mu}+g^{\mu \alpha} \delta_{\beta}^{\lambda},
$$

it is sufficient to specify operators $U_{a, \Lambda}$ for these special cases and then apply a group property.

In the "exact" theory, the operator $\mathcal{U}_{a, \Lambda}$ has the form

$$
\mathcal{U}_{a, \Lambda}=\exp \left[i \mathcal{P}^{0} a^{0}\right] \exp \left[-i \mathcal{P}^{j} a^{j}\right] \exp \left[i \alpha^{k} \mathcal{M}^{0 k}\right] \exp \left[\frac{i}{2} \mathcal{M}^{l m} \theta_{l m}\right]
$$

The momentum and angular momentum operators entering to formula (3.1) have the well-known form (see, for example, 25)

$$
\mathcal{P}^{\mu}=\int d \mathbf{x} T^{\mu 0}(\mathbf{x}), \quad \mathcal{M}^{\mu \lambda}=\int d \mathbf{x}\left[x^{\mu} T^{\lambda 0}(\mathbf{x})-x^{\lambda} T^{\mu 0}(\mathbf{x})\right],
$$

where formally

$$
T^{00}=\frac{1}{2} \hat{\pi}^{2}+\frac{1}{2} \partial_{i} \hat{\varphi} \partial_{i} \hat{\varphi}+\frac{m^{2}}{2} \hat{\varphi}^{2}+\frac{1}{\lambda} V(\sqrt{\lambda} \hat{\varphi}), \quad T^{k 0}=-\partial_{k} \hat{\varphi} \hat{\pi} .
$$

We are going to apply the operator (3.1) to the semiclassical state (1.3). Note that the operators $\mathcal{P}^{\mu}$ and $\mathcal{M}^{\mu \nu}(3.2)$ depend on field $\hat{\varphi}$ and momentum $\hat{\pi}$ semiclassically,

$$
\mathcal{P}^{\mu}=\frac{1}{\lambda} P^{\mu}(\sqrt{\lambda} \hat{\pi}(\cdot), \sqrt{\lambda} \hat{\varphi}(\cdot)), \quad \mathcal{M}^{\mu \nu}=\frac{1}{\lambda} M^{\mu \nu}(\sqrt{\lambda} \hat{\pi}(\cdot), \sqrt{\lambda} \hat{\varphi}(\cdot))
$$


It is convenient to consider the more general problem (cf. 35. . Let us find as $\lambda \rightarrow 0$ the state

$$
\exp (-i \mathcal{A}) K_{S^{0}, \Pi^{0}, \Phi^{0}} f^{0}
$$

where $K_{S, \Pi, \Phi}$ has the form (1.3),

$$
\mathcal{A}=\frac{1}{\lambda} A(\sqrt{\lambda} \hat{\pi}(\cdot), \sqrt{\lambda} \hat{\varphi}(\cdot))
$$

Note that the state functional (3.3) may be viewed as a solution to the Cauchy problem of the form

$$
\begin{gathered}
i \frac{\partial \Psi^{\tau}}{\partial \tau}=\frac{1}{\lambda} A\left(\frac{\sqrt{\lambda}}{i} \frac{\delta}{\delta \varphi(\cdot)}, \sqrt{\lambda} \varphi(\cdot)\right) \Psi^{\tau} \\
\Psi^{0}[\varphi(\cdot)]=\left(K_{S^{0}, \Pi^{0}, \Phi^{0}} f^{0}\right)[\varphi(\cdot)]
\end{gathered}
$$

at $\tau=1$. Let us look for the asymptotic solution to eq.(3.4) in the following form:

$$
\Psi^{\tau}[\varphi(\cdot)]=\left(K_{S^{\tau}, \Pi^{\tau}, \Phi^{\tau}} f^{\tau}\right)[\varphi(\cdot)]
$$

Substitution of functional (3.5) to eq.(3.4) gives us the following relation:

$$
\begin{gathered}
{\left[-\frac{1}{\lambda}\left(\dot{S}^{\tau}-\int d \mathbf{x} \Pi^{\tau}(\mathbf{x}) \dot{\Phi}^{\tau}(\mathbf{x})\right)-\frac{1}{\sqrt{\lambda}} \int d \mathbf{x}\left(\dot{\Pi}^{\tau}(\mathbf{x}) \phi(\mathbf{x})+\dot{\Phi}^{\tau}(\mathbf{x}) i \frac{\delta}{\delta \phi(\mathbf{x})}\right)+i \frac{\partial}{\partial \tau}\right] f^{\tau}[\phi(\cdot)]=} \\
\frac{1}{\lambda} A\left(\Pi^{\tau}(\cdot)-i \sqrt{\lambda} \frac{\delta}{\delta \phi(\cdot)}, \Phi^{\tau}(\cdot)+\sqrt{\lambda} \phi(\cdot)\right) f^{\tau}[\phi(\cdot)] .
\end{gathered}
$$

Considering the terms of the orders $O\left(\lambda^{-1}\right), O\left(\lambda^{-1 / 2}\right)$ and $O(1)$ in eq.(3.6), we obtain

$$
\begin{gathered}
\dot{S}^{\tau}=\int d \mathbf{x}\left(\Pi^{\tau}(\mathbf{x}) \dot{\Phi}^{\tau}(\mathbf{x})-A\left(\Pi^{\tau}(\cdot), \Phi^{\tau}(\cdot)\right)\right. \\
\dot{\Phi}^{\tau}(\mathbf{x})=\frac{\delta A\left(\Pi^{\tau}(\cdot), \Phi^{\tau}(\cdot)\right)}{\delta \Pi(\mathbf{x})}, \quad \dot{\Pi}^{\tau}(\mathbf{x})=-\frac{\delta A\left(\Pi^{\tau}(\cdot), \Phi^{\tau}(\cdot)\right)}{\delta \Phi(\mathbf{x})}, \\
\left.\left.i(\mathbf{x}) \frac{\delta^{2} A}{\delta \Phi(\mathbf{x}) \delta \Pi(\mathbf{y})} \frac{1}{i} \frac{\delta}{\delta \phi(\mathbf{y})}+\frac{1}{2} \phi(\mathbf{x}) \frac{\delta^{2} A}{\delta \Phi(\mathbf{x}) \delta \Phi(\mathbf{y})} \phi(\mathbf{y})\right]+A_{1}\right) f^{\tau}[\phi(\cdot)] .
\end{gathered}
$$

Here $A_{1}$ is a c-number quantity which depends on the ordering of the operators $\hat{\varphi}$ and $\hat{\pi}$ and is relevant to the renormalization problem.

We see that for the cases $\mathcal{A}=-\mathcal{P}^{0} a^{0}, \mathcal{A}=\mathcal{P}^{j} a^{j}, \mathcal{A}=-\alpha^{k} \mathcal{M}^{0 k}, \mathcal{A}=\frac{1}{2} \theta_{\text {sm }} \mathcal{M}^{s m}$ the mapping $u_{a, \Lambda}$ takes the initial condition for the system (3.7), (3.8) to the solution of the Cauchy problem for this system at $\tau=1$. The operators $\tilde{U}_{a, \Lambda}$ transforms the initial condition for eq.(3.9) to the solution at $\tau=1$.

2. The classical mappings $u_{a, \Lambda}$ for our partial cases are presented in table 1 .

One can write down the following general formula. Let $(a, \Lambda)$ be an arbitrary Poincare transformation. It happens that the mapping $u_{a, \Lambda}:(S, \Pi, \Phi) \mapsto(\tilde{S}, \tilde{\Pi}, \tilde{\Phi})$ has the following form. Let $\Phi(\mathbf{x}, t) \equiv \Phi(x)$ be a solution of the Cauchy problem

$$
\begin{gathered}
\partial_{\mu} \partial^{\mu} \Phi(x)+m^{2} \Phi(x)+V^{\prime}(\Phi(x))=0 \\
\Phi(\mathbf{x}, 0)=\Phi(\mathbf{x}),\left.\quad \frac{\partial}{\partial t} \Phi(\mathbf{x}, t)\right|_{t=0}=\Pi(\mathbf{x})
\end{gathered}
$$

Denote

$$
\breve{\Phi}(x)=\Phi\left(\Lambda^{-1}(x-a)\right) .
$$

It appears that

$$
\begin{gathered}
\tilde{\Phi}(\mathbf{x})=\breve{\Phi}(\mathbf{x}, 0), \quad \tilde{\Pi}(\mathbf{x})=\left.\frac{\partial}{\partial t} \breve{\Phi}(\mathbf{x}, t)\right|_{t=0}, \\
\tilde{S}=S+\int d x\left[\theta\left(x^{0}\right) \theta\left(-(\Lambda x+a)^{0}\right)-\theta\left(-x^{0}\right) \theta\left((\Lambda x+a)^{0}\right)\right] \\
\times\left[\frac{1}{2} \partial_{\mu} \Phi(x) \partial^{\mu} \Phi(x)-\frac{m^{2}}{2} \Phi^{2}(x)-V(\Phi(x)) .\right.
\end{gathered}
$$


Table 1: Poincare transformations in classical theory

\begin{tabular}{|c|c|c|}
\hline $\begin{array}{l}\text { Element of Poincare } \\
\text { group }\left(a_{\tau}, \Lambda_{\tau}\right)\end{array}$ & $\begin{array}{l}\text { Classical Poincare transformation } \\
\qquad u_{a_{\tau}, \Lambda_{\tau}}:\left(S^{0}, \Pi^{0}, \Phi^{0}\right) \mapsto\left(S^{\tau}, \Pi^{\tau}, \Phi^{\tau}\right)\end{array}$ & $\begin{array}{l}\text { Classical Lie derivative } \\
\qquad \delta F(S, \Pi, \Phi)=\left.\frac{d}{d \tau}\right|_{\tau=0} F\left(S^{\tau}, \Pi^{\tau}, \Phi^{\tau}\right)\end{array}$ \\
\hline $\begin{array}{l}a_{\tau}=0 \\
\Lambda_{\tau}=\exp \left(\frac{\tau}{2} l^{s m} \theta_{s m}\right) \\
\text { spatial rotation }\end{array}$ & $\begin{array}{c}\Phi^{\tau}(\mathbf{x})=\Phi^{0}\left(e^{-\frac{\tau}{2} l^{s m} \theta_{s m}} \mathbf{x}\right) \\
\Pi^{\tau}(\mathbf{x})=\Pi^{0}\left(e^{-\frac{\tau}{2} l^{s m} \theta_{s m}} \mathbf{x}\right) \\
S^{\tau}=S^{0}\end{array}$ & $\begin{array}{c}\frac{1}{2} \theta_{l m} \delta_{M}^{l m}= \\
\frac{1}{2} \theta_{l m} \int d \mathbf{x}\left(\left(x^{l} \partial_{m}-x^{m} \partial_{l}\right) \Phi(\mathbf{x}) \frac{\delta}{\delta \Phi(\mathbf{x})}\right. \\
\left.+\left(x^{l} \partial_{m}-x^{m} \partial_{l}\right) \Pi(\mathbf{x}) \frac{\delta}{\delta \Pi(\mathbf{x})}\right)\end{array}$ \\
\hline $\begin{array}{l}a_{\tau}^{0}=0, \Lambda_{\tau}=1 \\
\mathbf{a}_{\tau}=\mathbf{b} \tau \\
\text { spatial translation }\end{array}$ & $\begin{array}{c}\Phi^{\tau}(\mathbf{x})=\Phi^{0}(\mathbf{x}-\mathbf{b} \tau) \\
\Pi^{\tau}(\mathbf{x})=\Pi^{0}(\mathbf{x}-\mathbf{b} \tau) \\
S^{\tau}=S^{0}\end{array}$ & $\begin{array}{c}-b^{k} \delta_{P}^{k}= \\
-b^{k} \int d \mathbf{x}\left(\partial_{k} \Phi(\mathbf{x}) \frac{\delta}{\delta \Phi(\mathbf{x})}+\partial_{k} \Phi(\mathbf{x}) \frac{\delta}{\delta \Phi(\mathbf{x})}\right)\end{array}$ \\
\hline $\begin{array}{l}a^{0}=-\tau, \mathbf{a}=0 \\
\Lambda=1 \\
\text { evolution }\end{array}$ & $\begin{array}{l}\text { Resolving operator for the Cauchy } \\
\text { problem: } \\
\qquad \dot{\Phi}^{\tau}=\Pi^{\tau} \\
\dot{\Pi}^{\tau}=\left(-\Delta+m^{2}\right) \Phi^{\tau}+V^{\prime}\left(\Phi^{\tau}\right) \\
\dot{S}^{\tau}=\int d \mathbf{x}\left[\Pi^{\tau} \dot{\Phi}^{\tau}-\frac{1}{2}\left(\Pi^{\tau}\right)^{2}-\right. \\
\left.\frac{1}{2}\left(\nabla \Phi^{\tau}\right)^{2}-\frac{m^{2}}{2}\left(\Phi^{\tau}\right)^{2}-V\left(\Phi^{\tau}\right)\right]\end{array}$ & $\begin{array}{c}-\delta_{H}= \\
\int d \mathbf{x}\left[\Pi(\mathbf{x}) \frac{\delta}{\delta \Phi(\mathbf{x})}-(-\Delta \Phi(\mathbf{x})\right. \\
\left.\left.+m^{2} \Phi(\mathbf{x})+V^{\prime}(\Phi(\mathbf{x}))\right) \frac{\delta}{\delta \Pi(\mathbf{x})}\right]- \\
\int d \mathbf{x}\left[\frac{1}{2} \Pi^{2}(\mathbf{x})-\frac{1}{2}(\nabla \Phi(\mathbf{x}))^{2}\right. \\
\left.-\frac{m^{2}}{2} \Phi^{2}(\mathbf{x})-V(\Phi(\mathbf{x}))\right] \frac{\partial}{\partial S}\end{array}$ \\
\hline $\begin{array}{l}a_{\tau}=0 \\
\Lambda_{\tau}=\exp \left[-\tau n^{k} l^{0 k}\right] \\
\text { boost }\end{array}$ & $\begin{array}{l}\text { Resolving operator for the Cauchy } \\
\text { problem } \\
\qquad \dot{\Phi}^{\tau}=n^{k} x^{k} \Pi^{\tau} \\
-\dot{\Pi}^{\tau}=-\nabla x^{k} n^{k} \nabla \Phi^{\tau} \\
+x^{k} n^{k}\left(m^{2} \Phi^{\tau}+V^{\prime}\left(\Phi^{\tau}\right)\right) \\
\dot{S}^{\tau}=\int d \mathbf{x}\left[\Pi^{\tau} \dot{\Phi}^{\tau}-x^{k} n^{k}\left[\frac{1}{2}\left(\Pi^{\tau}\right)^{2}+\right.\right. \\
\left.\frac{1}{2}\left(\nabla \Phi^{\tau}\right)^{2}+\frac{m^{2}}{2}\left(\Phi^{\tau}\right)^{2}+V\left(\Phi^{\tau}\right)\right]\end{array}$ & $\begin{array}{c}-n^{m} \delta_{B}^{m}= \\
n^{m} \int d \mathbf{x}\left[x^{m} \Pi(\mathbf{x}) \frac{\delta}{\delta \Phi(\mathbf{x})}-\left(-\partial_{i} x^{m} \partial_{i} \Phi(\mathbf{x})\right.\right. \\
\left.\left.+x^{m} m^{2} \Phi(\mathbf{x})+x^{m} V^{\prime}(\Phi(\mathbf{x}))\right) \frac{\delta}{\delta \Pi(\mathbf{x})}\right]+ \\
n^{m} \int d \mathbf{x} x^{m}\left[\frac{1}{2} \Pi^{2}(\mathbf{x})-\frac{1}{2}(\nabla \Phi(\mathbf{x}))^{2}-\right. \\
\left.\frac{m^{2}}{2} \Phi^{2}(\mathbf{x})-V(\Phi(\mathbf{x}))\right] \frac{\partial}{\partial S}\end{array}$ \\
\hline
\end{tabular}


Table 2: Semiclassical Poincare transformations in functional representation

\begin{tabular}{|l|c|}
\hline $\begin{array}{l}\text { Element of Poincare } \\
\text { group }\left(a_{\tau}, \Lambda_{\tau}\right)\end{array}$ & $\begin{array}{c}\text { Semiclassical operator } \tilde{U}_{a_{\tau}, \Lambda_{\tau}}\left(u_{a_{\tau}, \Lambda_{\tau}} X \leftarrow X\right): f_{0} \mapsto f_{t} \text { in the functional repre- } \\
\text { sentation takes the initial condition for the Cauchy problem to the solution of } \\
\text { the Cauchy problem for the equation: }\end{array}$ \\
\hline $\begin{array}{l}a_{\tau}=0, \\
\begin{array}{l}\Lambda_{\tau}=\exp \left(\frac{\tau}{2} l^{s m} \theta_{s m}\right) ; \\
\text { spatial rotation }\end{array}\end{array}$ & $\begin{array}{c}i \dot{f}^{\tau}[\phi(\cdot)]=-\frac{1}{2} \theta_{s m} \tilde{M}^{s m}\left(X_{\tau}\right) f_{\tau}[\phi(\cdot)] ; \\
\tilde{M}^{s m}=-\int d \mathbf{x}\left[\left(x^{s} \partial_{m}-x^{m} \partial_{s}\right) \phi(\mathbf{x})\right] \frac{1}{i} \frac{\delta}{\delta \phi(\mathbf{x})} .\end{array}$ \\
\hline $\begin{array}{l}a_{\tau}^{0}=0, \Lambda_{\tau}=1, \\
\mathbf{a}_{\tau}=\mathbf{b} \tau ; \\
\text { spatial translation }\end{array}$ & $\begin{array}{c}i \dot{f}^{\tau}[\phi(\cdot)]=b^{k} \tilde{P}^{k}\left(X_{\tau}\right) f_{\tau}[\phi(\cdot)] ; \\
\tilde{P}^{k}=-\int d \mathbf{x} \partial_{k} \phi(\mathbf{x}) \frac{1}{i} \frac{\delta}{\delta \phi(\mathbf{x})} .\end{array}$ \\
\hline $\begin{array}{l}a^{0}=-\tau, \mathbf{a}=0 ; \\
\text { evolution }\end{array}$ & $\begin{array}{c}i \dot{f}^{\tau}[\phi(\cdot)]=\tilde{H}\left(X_{\tau}\right) f_{\tau}[\phi(\cdot)] ; \\
a_{\tau}=0 ; \\
\begin{array}{l}\Lambda_{\tau}=\exp \left[-\tau n^{k} l^{0 k}\right] ; \\
\text { boost }\end{array}\end{array}$ \\
\hline
\end{tabular}

For spatial translations, rotations and evolution, agreement between (3.11) and table 1 is evident. Consider the $x^{1}$-boost case, $n^{k}=(1,0, \ldots, 0)$. One has

$$
\begin{gathered}
\tilde{\Phi}_{\tau}(\mathbf{x})=\left.\Phi\left(x^{1} \cosh \tau+x^{0} \sinh \tau, x^{2}, \ldots, x^{d}, x^{0} \cosh \tau+x^{1} \sinh \tau\right)\right|_{x^{0}=0}, \\
\tilde{\Pi}_{\tau}(\mathbf{x})=\left.\frac{\partial}{\partial x^{0}} \Phi\left(x^{1} \cosh \tau+x^{0} \sinh \tau, x^{2}, \ldots, x^{d}, x^{0} \cosh \tau+x^{1} \sinh \tau\right)\right|_{x^{0}=0}
\end{gathered}
$$

The functions $\Phi_{\tau}, \Pi_{\tau}$ obey the system presented in table 1 . For the integral for $\tilde{S}$, consider the substitution $x^{0}=y^{1} \sinh \tilde{\tau}, x^{1}=y^{1} \cosh \tilde{\tau}, x^{2}=y^{2}, \ldots, x^{d}=y^{d}$. One finds

$$
\tilde{S}^{\tau}=S+\int_{0}^{\tau} d \tilde{\tau} y^{1} d \mathbf{y}\left[\frac{1}{2}\left(\tilde{\Pi}_{\tau}(\mathbf{y})\right)^{2}-\frac{1}{2}\left(\nabla \tilde{\Phi}_{\tau}(\mathbf{y})\right)^{2}-\frac{m^{2}}{2} \tilde{\Phi}_{\tau}^{2}(\mathbf{y})-V\left(\tilde{\Phi}_{\tau}(\mathbf{y})\right)\right]
$$

this agrees with table 1.

One can also notice that the group property for eq.(3.11) is satisfied.

Let us make more precise the definition of the space $\mathcal{X}$.

Definition 3.1. $\mathcal{X}$ is a space of sets $(S, \Pi, \Phi)$ of a number $S$ and functions $\Pi, \Phi \in S\left(\mathbf{R}^{d}\right)$ such that there exists a unique solution of the Cauchy problem (3.10) such that the functions $\left.\Phi(\Lambda x+a)\right|_{x^{0}=0}$ and $\left.\partial_{\mu} \Phi(\Lambda x+a)\right|_{x^{0}=0}$ are of the class $S\left(\mathbf{R}^{d}\right)$ for all a. $\Lambda$.

We see that the transformation $u_{a, \Lambda}: \mathcal{X} \rightarrow \mathcal{X}$ is defined.

3. The operators $\tilde{U}_{a, \Lambda}\left(u_{a, \Lambda} X \leftarrow X\right)$ are presented in table 2 .

However, it is not easy to check the group property (2.3). It is much more convenient to investigate the infinitesimal Poincare transformations and check the algebraic analog of (2.3).

It happens that operators $\tilde{U}_{a, \Lambda}\left(u_{a, \Lambda} X \leftarrow X\right)$ induce a Poincare group representation in a specific space. It is a space of sections $f(x ; \phi(\cdot))$ of the semiclassical bundle. The operators $\tilde{U}_{a, \Lambda}$ act as

$$
\left(\breve{\tilde{U}}_{a, \Lambda} f\right)(X)=\tilde{U}_{a, \Lambda}\left(X \leftarrow u_{a, \Lambda}^{-1} X\right) f\left(u_{a, \Lambda}^{-1} X\right) .
$$

The group property for the operators $\breve{\widetilde{U}}$ is equivalent to relation $(2.3)$. Let $\left(a_{t a u}, \Lambda_{\tau}\right)$ be a one-parametric subgroup of the Poincare group with the tangent vector $A$ being an element of the Poincare algebra. Since the operator $\tilde{U}_{a_{\tau}, \Lambda_{\tau}}\left(u_{a_{\tau}, \Lambda_{\tau}} X \leftarrow X\right)$ takes the initial condition for the cauchy problem for equation

$$
i \dot{f}_{\tau}=\tilde{H}\left(A: u_{a_{\tau}, \Lambda_{\tau}} X\right) f_{\tau}
$$


to the solution of this equation. therefore, the generator of representation $(3.12)$ is

$$
(\widetilde{\tilde{H}}(A) f)(X)=\left.i \frac{d}{d \tau}\right|_{\tau=0}\left(\widetilde{\tilde{U}}_{a_{\tau}, \Lambda_{\tau}} f\right)(X)=[\tilde{H}(A: X)-i \delta[A]] f(X)
$$

where

$$
\delta[A]=\left.\frac{d}{d \tau}\right|_{\tau=0} f\left(u_{a_{\tau}, \Lambda_{\tau}} X\right)
$$

is a Lie derivative presented in table 1. Therefore, the infinitesimal analog of the group property (2.3) is

$$
\left[\tilde{H}\left(A_{1}: X\right)-i \delta\left[A_{1}\right] ; \tilde{H}\left(A_{2}: X\right)-i \delta\left[A_{2}\right]\right]=i\left(\tilde{H}\left(\left[A_{1} ; A_{2}\right]: X\right)-i \delta\left[A_{1} ; A_{2}\right]\right) .
$$

It follows from notations of tables 1 and 2 that relation (3.13) can be rewritten for the Poincare algebra as

$$
\begin{gathered}
{\left[\breve{\tilde{P}}^{\lambda}, \breve{\widetilde{P}}^{\mu}\right]=0 ; \quad\left[\breve{\tilde{M}}^{\lambda \mu}, \breve{\tilde{P}}^{\sigma}\right]=i\left(g^{\mu \sigma} \breve{\tilde{P}}^{\lambda}-g^{\lambda \sigma} \breve{\widetilde{P}}^{\mu}\right)} \\
{\left[\breve{M}^{\lambda \mu}, \breve{M}^{\rho \sigma}\right]=-i\left(g^{\lambda \rho} \breve{M}^{\mu \sigma}-g^{\mu \rho} \breve{M}^{\lambda \sigma}+g^{\mu \sigma} \breve{M}^{\lambda \rho}-g^{\lambda \sigma} \breve{M}^{\mu \rho}\right) .}
\end{gathered}
$$

for operators

$$
\breve{\tilde{M}}^{m s}=\tilde{M}^{m s}+i \delta_{M}^{m s}, \quad \breve{\widetilde{P}}^{m}=\tilde{P}^{m}+i \delta_{P}^{m}, \quad \breve{\tilde{P}}^{0}=\tilde{H}+i \delta_{H}, \quad \breve{\tilde{M}}^{k 0}=\tilde{B}^{k}+i \delta_{B}^{k}
$$

It is checked by direct calculations that eqs.(3.14) are formally staisfied. However, there is a problem of divergences and renormalization which requires more careful investigations.

\subsection{Semiclassical Poincare transformations in Fock space}

For renormalization, let us construct the semiclassical Poincare transformations in the Fock space. They are related with the constructed operators $\tilde{U}_{a, \Lambda}\left(u_{a, \Lambda} X \leftarrow X\right)$ by the relation:

$$
\tilde{U}_{a, \Lambda}\left(u_{a, \Lambda} X \leftarrow X\right)=V_{u_{a, \Lambda} X} U_{a, \Lambda}\left(u_{a, \Lambda} X \leftarrow X\right) V_{X}^{-1}
$$

The operator $V$ taking the Fock space vector $\Psi \in \mathcal{F}$ to the functional $f[\phi(\cdot)]$ is defined from the relation

$$
V: \mid 0>\mapsto c \exp \left[\frac{i}{2} \int d \mathbf{x} d \mathbf{y} \tilde{\mathcal{R}}(\mathbf{x}, \mathbf{y}) \phi(\mathbf{x}) \phi(\mathbf{y})\right]
$$

and from formulas (2.1) which can be rewritten as

$$
\begin{aligned}
& V A^{+}(\mathbf{x}) V^{-1}=\mathcal{A}^{+}(\mathbf{x}) \equiv\left(\hat{\Gamma}^{-1 / 2} \hat{\mathcal{R}}^{*} \phi-\hat{\Gamma}^{-1 / 2} \frac{1}{i} \frac{\delta}{\delta \phi}\right)(\mathbf{x}), \\
& V A^{-}(\mathbf{x}) V^{-1}=\mathcal{A}^{-}(\mathbf{x}) \equiv\left(\hat{\Gamma}^{-1 / 2} \hat{\mathcal{R}} \phi-\hat{\Gamma}^{-1 / 2} \frac{1}{i} \frac{\delta}{\delta \phi}\right)(\mathbf{x}) .
\end{aligned}
$$

$|c|$ can be formally found from the normalization condition

$$
|c|^{2} \int D \phi\left|\exp \left[\frac{i}{2} \int d \mathbf{x} d \mathbf{y} \phi(\mathbf{x}) \tilde{\mathcal{R}}(\mathbf{x}, \mathbf{y}) \phi(\mathbf{y})\right]\right|^{2}=1
$$

The argument can be chosen to be arbitrary, for example,

$$
\operatorname{Argc}=0
$$

Notice that the operator $V$ is defined form the relations (3.16) - (3.19) uniquely.

Namely, any element of the Fock space can be presented 42] via its components, vacuum state an creation operators as

$$
\Psi=\sum_{n=0}^{\infty} \frac{1}{\sqrt{n !}} \int d \mathbf{x}_{1} \ldots d \mathbf{x}_{n} \Psi_{n}\left(\mathbf{x}_{1}, \ldots, \mathbf{x}_{n}\right) A^{+}\left(\mathbf{x}_{1}\right) \ldots A^{+}\left(\mathbf{x}_{n}\right) \mid 0>
$$


Specify

$$
V \Psi=\sum_{n=0}^{\infty} \frac{1}{\sqrt{n !}} \int d \mathbf{x}_{1} \ldots d \mathbf{x}_{n} \Psi_{n}\left(\mathbf{x}_{1}, \ldots, \mathbf{x}_{n}\right) \mathcal{A}^{+}\left(\mathbf{x}_{1}\right) \ldots \mathcal{A}^{+}\left(\mathbf{x}_{n}\right) V \mid 0>.
$$

The problem of divergence of the series is related with the problem of correctness of the functional Schrodinger representation. It is not investigated here.

Since the operators $\mathcal{A}^{ \pm}(\mathbf{x})$ satisfy usual canonical commutation relations and $\mathcal{A}^{-}(\mathbf{x}) \mid 0>=0$, we obtain $V A^{ \pm}(\mathbf{x})=\mathcal{A}^{ \pm}(\mathbf{x}) V$.

The operator $V$ depend on $\mathcal{R}$. It is useful to find an explicit form of the operator $V^{-1} \delta V$.

It happens that the following property is satisfied:

$$
\begin{gathered}
V^{-1} \delta V=-\frac{i}{2} A^{+} \hat{\Gamma}^{-1 / 2} \delta \hat{\mathcal{R}} \hat{\Gamma}^{-1 / 2} A^{+}-\frac{i}{2} A^{-} \hat{\Gamma}^{-1 / 2} \delta \hat{\mathcal{R}}^{*} \hat{\Gamma}^{-1 / 2} A^{-}+ \\
A^{+}\left[\hat{\Gamma}^{1 / 2} \delta \hat{\Gamma}^{-1 / 2}+i \hat{\Gamma}^{-1 / 2} \delta \hat{\mathcal{R}}^{*} \hat{\Gamma}^{-1 / 2}\right] A^{-}+\frac{i}{4} \operatorname{Tr}\left[\delta\left(\hat{\mathcal{R}}+\hat{\mathcal{R}}^{*}\right) \hat{\Gamma}^{-1}\right]
\end{gathered}
$$

The notations of the type $A^{+} \hat{\mathcal{B}} A^{-}$are used for the operators like $\int d \mathbf{x} d \mathbf{y} A^{+}(\mathbf{x}) \tilde{\mathcal{B}}(\mathbf{x}, \mathbf{y}) A^{-}(\mathbf{y})$, where $\tilde{\mathcal{B}}(\mathbf{x}, \mathbf{y})$ is a kernel of the operator $\hat{\mathcal{B}}$.

To check formula (3.20), consider the variation of the formula (2.1) if $\mathcal{R}$ is varied:

$$
\begin{gathered}
{\left[A^{ \pm}(\mathbf{x}) ; V^{-1} \delta V\right]=\left(\hat{\Gamma}^{1 / 2} \delta \hat{\Gamma}^{-1 / 2} A^{ \pm}\right)(\mathbf{x})} \\
-i\left(\hat{\Gamma}^{-1 / 2} \delta \hat{\mathcal{R}} \hat{\Gamma}^{-1 / 2} A^{+}\right)(\mathbf{x})+i\left(\hat{\Gamma}^{-1 / 2} \delta \hat{\mathcal{R}}^{*} \hat{\Gamma}^{-1 / 2} A^{-}\right)(\mathbf{x}) .
\end{gathered}
$$

Therefore, formula (3.20) is correct up to an additive constant. To find it, note that

$$
\delta V\left|0>=\left[\frac{i}{2} \int d \mathbf{x} d \mathbf{y} \phi(\mathbf{x}) \delta \tilde{\mathcal{R}}(\mathbf{x}, \mathbf{y}) \phi(\mathbf{y})+\delta \ln c\right] V\right| 0>.
$$

This relation and formula (2.1) imply

$$
<0\left|V^{-1} \delta V\right| 0>=\frac{i}{2} \operatorname{Tr}\left(\delta \hat{\mathcal{R}} \hat{\Gamma}^{-1}\right)+\delta \ln c .
$$

It follows from the normalization conditions $(3.18)$ and $(3.19)$ that $c=(\operatorname{det} \hat{\Gamma})^{1 / 4}$. Therefore, $\delta \ln c=$ $\frac{1}{4} \operatorname{Tr} \delta \hat{\Gamma} \hat{\Gamma}^{-1}$. Thus, $<0\left|V^{-1} \delta V\right| 0>=\frac{i}{4} \operatorname{Tr} \delta\left(\hat{\mathcal{R}}+\hat{\mathcal{R}}^{*}\right) \Gamma^{-1}$. Formula (3.20) is checked.

It follows from formula (3.15) that the generators $H(A: X)$ in the Fock representation are related with $\tilde{H}(A: X)$ by the following relation:

$$
\breve{H}(A: X)=H(A: X)-i \delta[A]=V_{X}^{-1}(\tilde{H}[A: X]-i \delta[A]) V_{X} .
$$

We see that commutation relations (3.13) are invariant under change of representation.

An explicit form of operators $H(A: X)$ will be simplified if we consider the case when the quadratic form $\mathcal{R}$ is invariant under spatial translations and rotations:

$$
\tilde{\mathcal{R}}\left(\mathbf{x}, \mathbf{y}: u_{(\mathbf{a}, L)} X\right)=\tilde{\mathcal{R}}\left(L^{-1}(\mathbf{x}-\mathbf{a}), L^{-1}(\mathbf{y}-\mathbf{a}): X\right) .
$$

This property implies that

$$
\begin{aligned}
{\left[\partial_{k} ; \hat{\mathcal{R}}\right]=\delta_{P}^{k} \hat{\mathcal{R}} ; } & {\left[\partial_{k} ; \hat{\Gamma}^{1 / 2}\right]=\delta_{P}^{k} \hat{\Gamma}^{1 / 2} ; } \\
{\left[\left(x^{k} \partial_{l}-x^{l} \partial_{k}\right) ; \hat{\mathcal{R}}\right]=\delta_{M}^{k l} \hat{\mathcal{R}} ; } & {\left[\left(x^{k} \partial_{l}-x^{l} \partial_{k}\right) ; \hat{\Gamma}^{1 / 2}\right]=\delta_{M}^{k l} \hat{\Gamma}^{1 / 2} . }
\end{aligned}
$$

The generators $H(A: X)$ are presented in table 3 .

We see that renormalization is necessary since the evolution and boost generators contain divergent terms $\frac{1}{4} \operatorname{Tr} \hat{\Gamma}$ and $\frac{1}{4} \operatorname{Tr} x^{k} \hat{\Gamma}$ which are to be changed by finite renormalizaed terms $\frac{1}{4} \operatorname{Tr}_{R} \hat{\Gamma}$ and $\frac{1}{4} \operatorname{Tr}_{R} x^{k} \hat{\Gamma}$.

Let us check the commutation relations between $\breve{H}(A: X)$. Since the divergences arise in terms $\overline{B^{k}}$ and $\bar{H}$ only, so that we suppose them to be arbitrary and then find the conditions that provide Poincare invariance. 
Table 3: Semiclassical Poincare transformations in Fock representation

\begin{tabular}{|c|c|}
\hline $\begin{array}{l}\text { Element of Poincare } \\
\text { group }\left(a_{\tau}, \Lambda_{\tau}\right)\end{array}$ & $\begin{array}{l}\text { Semiclassical operator } U_{a_{\tau}, \Lambda_{\tau}}\left(u_{a_{\tau}, \Lambda_{\tau}} X \leftarrow X\right): \Psi_{0} \mapsto \Psi_{t} \text { in the Fock represen- } \\
\text { tation takes the initial condition for the Cauchy problem to the solution of the } \\
\text { Cauchy problem for the equation: }\end{array}$ \\
\hline $\begin{array}{l}a_{\tau}=0 \\
\Lambda_{\tau}=\exp \left(\frac{\tau}{2} l^{s m} \theta_{s m}\right) \\
\text { spatial rotation }\end{array}$ & $\begin{array}{c}i \dot{\Psi}^{\tau}=-\frac{1}{2} \theta_{s m} M^{s m} \Psi_{\tau} \\
M^{k l}=-i A^{+}\left(x^{k} \partial_{l}-x^{l} \partial_{k}\right) A^{-}\end{array}$ \\
\hline $\begin{array}{l}a_{\tau}^{0}=0, \Lambda_{\tau}=1 \\
\mathbf{a}_{\tau}=\mathbf{b} \tau \\
\text { spatial translation }\end{array}$ & $\begin{array}{c}i \dot{\Psi}^{\tau}=b^{k} P^{k} \Psi_{\tau} \\
P^{k}=-i A^{+} \partial_{k} A^{-}\end{array}$ \\
\hline $\begin{array}{l}a^{0}=-\tau, \mathbf{a}=0 \\
\Lambda=1 \\
\text { evolution }\end{array}$ & $\begin{array}{c}i \dot{\Psi}^{\tau}=H\left(X_{\tau}\right) \Psi_{\tau} \\
H(X)=\frac{1}{2} A^{-} \mathcal{H}^{--}(X) A^{-}+A^{+}(\hat{\omega}+\mathcal{H}(X)) A^{-}+\frac{1}{2} A^{+} \mathcal{H}^{++}(X) A^{+}+\bar{H} \\
\mathcal{H}^{++}(X)=\hat{\Gamma}^{-1 / 2}\left[\delta_{H} \hat{\mathcal{R}}-\hat{\mathcal{R}} \hat{\mathcal{R}}-\left(-\Delta+m^{2}+V^{\prime \prime}(\Phi(\mathbf{x}))\right] \hat{\Gamma}^{-1 / 2}\right. \\
\mathcal{H}^{--}(X)=\left(\mathcal{H}^{++}\right)^{+} ; \\
\mathcal{H}(X)=\hat{\Gamma}^{-1 / 2}\left(\hat{\mathcal{R}} \hat{\mathcal{R}}^{*}+\left(-\Delta+m^{2}+V^{\prime \prime}(\Phi(\mathbf{x}))-\frac{1}{2} \delta_{H}\left(\hat{\mathcal{R}}+\hat{\mathcal{R}}^{*}\right)\right.\right. \\
\left.+\frac{i}{2}\left[\delta_{H} \hat{\Gamma}^{1 / 2} ; \hat{\Gamma}^{1 / 2}\right]\right) \hat{\Gamma}^{-1 / 2}-\hat{\omega} \\
\hat{\omega}=\sqrt{-\Delta+m^{2}} \\
\text { formally } \quad \bar{H}=\bar{H}_{r e g}+\frac{1}{4} \operatorname{Tr} \hat{\Gamma} \\
\bar{H}_{\text {reg }}=-\frac{1}{4} \operatorname{Tr}\left[\mathcal{H}^{++}+\mathcal{H}^{--}\right] .\end{array}$ \\
\hline $\begin{array}{l}a_{\tau}=0 \\
\Lambda_{\tau}=\exp \left[-\tau n^{k} l^{0 k}\right] \\
\text { boost }\end{array}$ & $\begin{array}{c}i \dot{\Psi}^{\tau}=n^{m} B^{m}\left(X_{\tau}\right) \Psi_{\tau} \\
B^{k}(X)=\frac{1}{2} A^{-} \mathcal{B}^{k--}(X) A^{-}+A^{+}\left(L_{k}+\mathcal{B}^{k}(X)\right) A^{-}+\frac{1}{2} A^{+} \mathcal{B}^{k++}(X) A^{+}+\overline{B^{k}} \\
\mathcal{B}^{k++}(X)=\hat{\Gamma}^{-1 / 2}\left[\delta_{k}^{B} \hat{\mathcal{R}}-\hat{\mathcal{R}} x^{k} \hat{\mathcal{R}}-\left(-\partial_{i} x^{k} \partial_{i}+x^{k} m^{2}+x^{k} V^{\prime \prime}(\Phi(\mathbf{x}))\right] \hat{\Gamma}^{-1 / 2}\right. \\
\mathcal{B}^{k--}=\left(\mathcal{B}^{k++}\right)^{+} \\
\mathcal{B}^{k}=\hat{\Gamma}^{-1 / 2}\left[\hat{\mathcal{R}} x^{k} \hat{\mathcal{R}}^{*}+\left(-\partial_{i} x^{k} \partial_{i}+x^{k} m^{2}+x^{k} V^{\prime \prime}(\Phi(\mathbf{x}))\right)-\frac{1}{2} \delta_{B}^{k}\left(\hat{\mathcal{R}}+\hat{\mathcal{R}}^{*}\right)+\right. \\
\left.\frac{i}{2}\left[\delta_{k}^{B} \hat{\Gamma}^{1 / 2}, \hat{\Gamma}^{1 / 2}\right]\right] \hat{\Gamma}^{-1 / 2}-L_{k} ; \\
L_{k}=\frac{1}{2} \hat{\omega}^{-1 / 2}\left[\hat{\omega} x^{k} \hat{\omega}+\left(-\partial_{i} x^{k} \partial_{i}+x^{k} m^{2}\right)\right] \hat{\omega}^{-1 / 2} \\
\text { formally } \overline{B^{k}}=\bar{B}_{r e g}+\frac{1}{4} \operatorname{Tr} x^{k} \hat{\Gamma} ; \\
\bar{B}_{r e g}^{k}=-\frac{1}{4} \operatorname{Tr}\left[\mathcal{B}^{k++}+\mathcal{B}^{k--}\right] .\end{array}$ \\
\hline
\end{tabular}


Let

$$
\breve{H}_{k}=\frac{1}{2} A^{+} \mathcal{H}_{k}^{++} A^{+}+A^{+} \mathcal{H}^{+-} A^{-}+\frac{1}{2} A^{-} \mathcal{H}^{--} A^{-}+\overline{H_{k}}+i \delta_{k}
$$

be arbitrary quadratic Hamiltonians. Then the property $\left[\breve{H}_{1}, \breve{H}_{2}\right]=i \breve{H}_{3}$ under condition $\left[i \delta_{1}, i \delta_{2}\right]=i^{2} \delta_{3}$ means that

$$
\begin{gathered}
\mathcal{H}_{3}^{++}=-i\left[\mathcal{H}_{1}^{+-} \mathcal{H}_{2}^{++}+\mathcal{H}_{2}^{++}\left(\mathcal{H}_{1}^{+-}\right)^{*}-\mathcal{H}_{1}^{++}\left(\mathcal{H}_{2}^{+-}\right)^{*}-\mathcal{H}_{2}^{+-} \mathcal{H}_{1}^{++}\right]+\delta_{1} \mathcal{H}_{2}^{++}-\delta_{2} \mathcal{H}_{1}^{++} . \\
\mathcal{H}_{3}^{+-}=-i\left\{\mathcal{H}_{2}^{++}\left(\mathcal{H}_{1}^{++}\right)^{*}-\mathcal{H}_{1}^{++}\left(\mathcal{H}_{2}^{++}\right)^{*}+\left[\mathcal{H}_{1}^{+-} ;\left(\mathcal{H}_{2}^{+-}\right)\right]\right\}+\delta_{1} \mathcal{H}_{2}^{+-}-\delta_{2} \mathcal{H}_{1}^{+-}, \\
\overline{H_{3}}=-\frac{i}{2} \operatorname{Tr}\left[\mathcal{H}_{2}^{++}\left(\mathcal{H}_{1}^{++}\right)^{*}-\mathcal{H}_{1}^{++}\left(\mathcal{H}_{2}^{++}\right)^{*}\right]+\delta_{1} \overline{H_{2}}-\delta_{2} \overline{H_{1}} .
\end{gathered}
$$

Relations (3.23), (3.24), (3.25) are treated in sense of bilinear forms on $D(T)$.

Consider now the commutation relations.

1. The relations

$$
\left[\breve{P}^{k}, \breve{P}^{l}\right]=0, \quad\left[\breve{M}^{l m}, \breve{P}^{s}\right]=i\left(g^{m s} \breve{P}^{l}-g^{l s} \breve{P}^{m}\right]
$$

are satisfied automatically since

$$
\left[\partial_{k}, \partial_{l}\right]=0, \quad-\left[x^{l} \partial_{m}-x^{m} \partial_{l}, \partial_{s}\right]=g^{m s} \partial_{l}-g^{l s} \partial_{m} .
$$

2. The relation

$$
\left[\breve{M}^{l m}, \breve{M}^{r s}\right]=-i\left(g^{l r} \breve{M}^{m s}-g^{m r} \breve{M}^{l s}+g^{m s} \breve{M}^{l r}-g^{l s} \breve{M}^{m r}\right)
$$

is also satisfied.

3. For the relation

$$
\left[\breve{P}^{k}, \breve{P}^{0}\right]=0
$$

eqs (3.23)- (3.25) takes the form

$$
\begin{gathered}
\delta_{P}^{k} \mathcal{H}^{++}-\left[\partial_{k} ; \mathcal{H}^{++}\right]=0, \quad \delta_{P}^{k} \mathcal{H}^{+-}-\left[\partial_{k} ; \mathcal{H}^{+-}\right]=0, \\
\delta_{P}^{k} \bar{H}=0 .
\end{gathered}
$$

4. For the relation

$$
\left[\breve{M}^{k l}, \breve{P}^{0}\right]=0
$$

eqs. (3.23) - (3.25) are written as

$$
\begin{gathered}
\delta_{M}^{k l} \mathcal{H}^{++}-\left[x^{k} \partial_{l}-x^{l} \partial_{k} ; \mathcal{H}^{++}\right]=0 ; \quad \delta_{M}^{k l} \mathcal{H}^{+-}-\left[x^{k} \partial_{l}-x^{l} \partial_{k} ; \mathcal{H}^{+-}\right]=0 ; \\
\delta_{M}^{k l} \bar{H}=0 .
\end{gathered}
$$

5. Consider the relation

$$
\left[\breve{M}^{k 0}, \breve{P}^{s}\right]=-i g^{k s} \breve{P}^{0}
$$

We write eqs.(3.23) - (3.25) as follows:

$$
\begin{gathered}
{\left[\partial_{s}, \mathcal{B}^{k++}\right]-\delta_{P}^{s} \mathcal{B}^{k++}=-g^{k s} \mathcal{H}^{++}, \quad\left[\partial_{s}, \mathcal{B}^{k+-}\right]-\delta_{P}^{s} \mathcal{B}^{k+-}=-g^{k s} \mathcal{H}^{+-},} \\
\delta_{P}^{s} \overline{B^{k}}=g^{k s} \bar{H} .
\end{gathered}
$$

6. The commutation relation

$$
\left[\breve{M}^{l m}, \breve{M}^{k 0}\right]=-i\left(g^{l k} \breve{M}^{m 0}-g^{m k} \breve{M}^{l 0}\right)
$$

is equivalent to

$$
\left[x^{l} \partial_{m}-x^{m} \partial_{l} ; \mathcal{B}^{k++}\right]-\delta_{M}^{l m} \mathcal{B}^{k++}=g^{l k} \mathcal{B}^{m++}-g^{m k} \mathcal{B}^{l++},
$$




$$
\begin{gathered}
{\left[x^{l} \partial_{m}-x^{m} \partial_{l} ; \mathcal{B}^{k+-}\right]-\delta_{M}^{l m} \mathcal{B}^{k+-}=g^{l k} \mathcal{B}^{m+-}-g^{m k} \mathcal{B}^{l+-},} \\
-\delta_{M}^{k l} \overline{B^{k}}=g^{l k} \overline{B^{m}}-g^{m k} \overline{B^{l}}
\end{gathered}
$$

7. The most nontrivial commutation relations are

$$
\left[\breve{M}^{k 0} ; \breve{P}^{0}\right]=i \breve{P}^{k}, \quad\left[\breve{M}^{k 0} ; \breve{M}^{l 0}\right]=-i \breve{M}^{k l}
$$

They can be rewritten as follows:

$$
\begin{gathered}
0=-i\left\{\mathcal{B}^{k+-} \mathcal{H}^{++}+\mathcal{H}^{++}\left(\mathcal{B}^{k+-}\right)^{*}-\mathcal{B}^{k++}\left(\mathcal{H}^{+-}\right)^{*}-\mathcal{H}^{+-}\left(\mathcal{B}^{k++}\right)\right\}+\delta_{B}^{k} \mathcal{H}^{++}-\delta_{H} \mathcal{B}^{k++} \\
-i \partial_{k}=-i\left\{\mathcal{H}^{++} \mathcal{B}^{k++}-\mathcal{B}^{k++}\left(\mathcal{H}^{++}\right)^{*}+\left[\mathcal{B}^{k+-} ; \mathcal{H}^{+-}\right]\right\}+\delta_{B}^{k} \mathcal{H}^{+-}-\delta_{H} \mathcal{B}^{k+-} \\
0=-\frac{i}{2} \operatorname{Tr}\left[\mathcal{H}^{++}\left(\mathcal{B}^{k++}\right)^{*}-\mathcal{B}^{k++}\left(\mathcal{H}^{++}\right)^{*}\right]+\delta_{B}^{k} \bar{H}-\delta_{H} \overline{B^{k}}
\end{gathered}
$$

and

$$
\begin{gathered}
0=-i\left\{\mathcal{B}^{k+-} \mathcal{B}^{l++}+\mathcal{B}^{l++}\left(\mathcal{B}^{k+-}\right)^{*}-\mathcal{B}^{k++}\left(\mathcal{B}^{l+-}\right)^{*}-\mathcal{B}^{l+-}\left(\mathcal{B}^{k++}\right)\right\}+\delta_{B}^{k} \mathcal{B}^{l++}-\delta_{B}^{l} \mathcal{B}^{k++} \\
i\left(x^{k} \partial_{l}-x^{l} \partial_{k}\right)=-i\left\{\mathcal{B}^{l++} \mathcal{B}^{k++}-\mathcal{B}^{k++}\left(\mathcal{B}^{l++}\right)^{*}+\left[\mathcal{B}^{k+-} ; \mathcal{B}^{l+-}\right]\right\}+\delta_{B}^{k} \mathcal{B}^{l+-}-\delta_{B}^{l} \mathcal{B}^{k+-} \\
0=-\frac{i}{2} \operatorname{Tr}\left[\mathcal{B}^{l++}\left(\mathcal{B}^{k++}\right)^{*}-\mathcal{B}^{k++}\left(\mathcal{B}^{l++}\right)^{*}\right]+\delta_{B}^{k} \overline{B^{l}}-\delta_{B}^{l} \overline{B^{k}}
\end{gathered}
$$

3. Properties (3.26), (3.28), (3.30), (3.32) are obvious corollaries of relations (3.22). Properties (3.34) and (3.36) are checked by nontrivial but also direct computations.

Properties (3.27), (3.29), (3.31), (3.33), 3.35), (3.37) will be satisfied if the renormalized trace satisifies the following properties:

$$
\begin{gathered}
\delta_{k}^{P} \operatorname{Tr}_{R} \hat{\Gamma}=0 ; \quad \delta_{k l}^{M} \operatorname{Tr}_{R} \hat{\Gamma}=0 ; \\
\delta_{l}^{P} \operatorname{Tr}_{R} x^{k} \hat{\Gamma}=-\delta^{k l} \operatorname{Tr}_{R} \hat{\Gamma} ; \quad \delta_{k l}^{M} \operatorname{Tr}_{R} x^{k} \hat{\Gamma}=\delta^{k l} \operatorname{Tr}_{R} x^{m} \hat{\Gamma}-\delta^{m k} \operatorname{Tr}_{R} x^{l} \hat{\Gamma} ; \\
\operatorname{Tr}\left[x^{l}\left(\delta_{k}^{B} \hat{\Gamma}-\hat{\mathcal{A}} x^{k} \hat{\Gamma}-\hat{\Gamma} x^{k} \hat{\mathcal{A}}\right)-x^{k}\left(\delta_{l}^{B} \hat{\Gamma}-\hat{\mathcal{A}} x^{l} \hat{\Gamma}-\hat{\Gamma} x^{l} \hat{\mathcal{A}}\right)\right]+\delta_{l}^{B} \operatorname{Tr}_{R} x^{k} \hat{\Gamma}-\delta_{k}^{B} \operatorname{Tr}_{R} x^{l} \hat{\Gamma}=0 ; \\
\operatorname{Tr}\left[x^{l}\left(\delta^{H} \hat{\Gamma}-\hat{\mathcal{A}} \hat{\Gamma}-\hat{\Gamma} \hat{\mathcal{A}}\right)-\left(\delta_{l}^{B} \hat{\Gamma}-\hat{\mathcal{A}} x^{l} \hat{\Gamma}-\hat{\Gamma} x^{l} \hat{\mathcal{A}}\right)\right]+\delta_{l}^{B} \operatorname{Tr}_{R} \hat{\Gamma}-\delta^{H} \operatorname{Tr}_{R} x^{l} \hat{\Gamma}=0,
\end{gathered}
$$

where $\hat{\mathcal{A}}=\frac{1}{2}\left(\hat{\mathcal{R}}+\hat{\mathcal{R}}^{*}\right)$.

Thus, algebraic commutation relations are checked.

\subsection{Conditions of integrability}

The problem of reconstructing a representation of a local Lie group from a representation of a Lie algebra ("integrability problem") is mathematically nontrivial. Different conditions of integrability were presented in [43, 44, 45, 46, 47].

The problem of reconstructing the operators $U_{g}\left(u_{g} X \leftarrow X\right)$ and checking the group property was discussed in details in 48]. It has been shown that the operators $U_{g}\left(u_{g} X \leftarrow X\right)$ are correctly defined under the following sufficient conditions.

Let $h(\alpha)$ be an arbitrary smooth curve on the Poincare group.

P1, For self-adjoint operators

$$
A_{k}=L_{k}, \quad A_{d+k}=-i \partial_{k}, \quad A_{2 d+k d+l}=-i\left(x^{k} \partial_{l}-x^{l} \partial_{k}\right), \quad A_{2 d+d^{2}+1}=\hat{\omega}
$$

there exists such a positively definite operator $T$ that

1. $\left\|T^{-1 / 2} A_{j} T^{-1 / 2}\right\|<\infty,\left\|A_{j} T^{-1}\right\|<\infty$.

2. for all $t_{1}$ there exists such a constant $C$ that $\left\|T^{1 / 2} e^{i A_{j} t} T^{-1 / 2}\right\| \leq C,\left\|T e^{-i A_{j} t} T^{-1}\right\| \leq C, t \in\left[-t_{1}, t_{1}\right]$.

P2. The $\alpha$-dependent operator functions $\operatorname{TB}^{k++}\left(u_{h(\alpha)} X\right)$ and $T \mathcal{H}^{++}\left(u_{h(\alpha)} X\right)$ are continuous in the Hilbert-Schmidt topology $\|\cdot\|_{2}$. 
P3. The $\alpha$-dependent operator functions $\mathcal{B}^{k++}\left(u_{h(\alpha)} X\right)$ and $\mathcal{H}^{++}\left(u_{h(\alpha)} X\right)$ are continuously differentiable with respect to $\alpha$ in the Hilbert-Schmidt topology.

$\mathrm{P} 4$.

The

$\alpha$-dependent operator functions $\mathcal{B}^{k}\left(u_{h(\alpha)} X\right), \mathcal{H}\left(u_{h(\alpha)} X\right), T \mathcal{B}^{k}\left(u_{h(\alpha)} X\right) T^{-1}, T^{1 / 2} \mathcal{B}^{k}\left(u_{h(\alpha)} X\right) T^{-1 / 2}, T \mathcal{H}\left(u_{h(\alpha)} X\right) T^{-1}$, $T^{1 / 2} \mathcal{H}\left(u_{h(\alpha)} X\right) T^{-1 / 2}$ are strongly continuous.

P5. The $\alpha$-dependent operator functions $T^{-1 / 2} \mathcal{H}\left(u_{h(\alpha)} X\right) T^{-1 / 2}, \quad T^{-1 / 2} \mathcal{B}^{k}\left(u_{h(\alpha)} X\right) T^{-1 / 2}$, $\mathcal{H}\left(u_{h(\alpha)} X\right) T^{-1}, \mathcal{B}^{k}\left(u_{h(\alpha)} X\right) T^{-1}$ are continuously differentiable with respect to $\alpha$ in the operator norm $\|\cdot\|$ topology.

P6. The functions $\bar{H}\left(u_{h(\alpha)} X\right)$ and $\overline{B^{k}}\left(u_{h(\alpha)} X\right)$ are continuous.

The property $\mathrm{P} 6$ can be substituted by the following property.

P6'. (a) The operators $\mathcal{B}^{k++}$ and $\mathcal{H}^{++}$are of the trace class and $\operatorname{Tr} \mathcal{B}^{k++}\left(u_{h(\alpha)} X\right)$ and $\operatorname{Tr} \mathcal{H}^{++}\left(u_{h(\alpha)} X\right)$ are continuous functions of $\alpha$.

(b) The functions $\operatorname{Tr}_{R} \Gamma\left(u_{h(\alpha)} X\right)$ and $\operatorname{Tr}_{R} x^{k} \Gamma\left(u_{h(\alpha)} X\right)$ are continuous.

Let us first justify property $\mathrm{P} 1$.

Let

$$
\hat{K}=\hat{\omega}^{-1 / 4}\left(\mathbf{x}^{2}+1\right)^{-1} \hat{\omega}^{-1 / 4}
$$

This is a bounded self-adjoint positively definite operator without zero eigenvalues. Therefore, $\hat{K}^{-1} \equiv$ $T^{1 / 2}$ is a (non-bounded) self-adjoint operator and

$$
T=\hat{\omega}^{1 / 4}\left(\mathbf{x}^{2}+1\right) \hat{\omega}^{1 / 2}\left(\mathbf{x}^{2}+1\right) \hat{\omega}^{1 / 4}
$$

$T \geq c>0$ for some $c$.

The first part of property P1 is justified as follows. One should check that the following norms are finite:

$$
\begin{gathered}
\left\|\hat{\omega}^{-1 / 4}\left(\mathbf{x}^{2}+1\right)^{-1} \hat{\omega}^{-1 / 4} \hat{\omega} \hat{\omega}^{-1 / 4}\left(\mathbf{x}^{2}+1\right)^{-1} \hat{\omega}^{-1 / 4}\right\| ; \\
\left\|\hat{\omega}^{-1 / 4}\left(\mathbf{x}^{2}+1\right)^{-1} \hat{\omega}^{-1 / 4} \hat{\omega} x^{s} \hat{\omega}^{-1 / 4}\left(\mathbf{x}^{2}+1\right)^{-1} \hat{\omega}^{-1 / 4}\right\| ; \\
\left\|\hat{\omega}^{-1 / 4}\left(\mathbf{x}^{2}+1\right)^{-1} \hat{\omega}^{-1 / 4}\left(\hat{k}^{j} x^{s}-\hat{k}^{s} x^{j}\right) \hat{\omega}^{-1 / 4}\left(\mathbf{x}^{2}+1\right)^{-1} \hat{\omega}^{-1 / 4}\right\| ; \\
\left\|\hat{\omega}^{-1 / 4}\left(\mathbf{x}^{2}+1\right)^{-1} \hat{\omega}^{-1 / 4} \hat{k}^{j} \hat{\omega}^{-1 / 4}\left(\mathbf{x}^{2}+1\right)^{-1} \hat{\omega}^{-1 / 4}\right\| ; \\
\left\|\hat{\omega} \hat{\omega}^{-1 / 4}\left(\mathbf{x}^{2}+1\right)^{-1} \hat{\omega}^{-1 / 4} \hat{\omega}^{-1 / 4}\left(\mathbf{x}^{2}+1\right)^{-1} \hat{\omega}^{-1 / 4}\right\| ; \\
\left\|\hat{\omega} x^{s} \hat{\omega}^{-1 / 4}\left(\mathbf{x}^{2}+1\right)^{-1} \hat{\omega}^{-1 / 4} \hat{\omega}^{-1 / 4}\left(\mathbf{x}^{2}+1\right)^{-1} \hat{\omega}^{-1 / 4}\right\| ; \\
\left\|\left(\hat{k}^{j} x^{s}-\hat{k}^{s} x^{j}\right) \hat{\omega}^{-1 / 4}\left(\mathbf{x}^{2}+1\right)^{-1} \hat{\omega}^{-1 / 4} \hat{\omega}^{-1 / 4}\left(\mathbf{x}^{2}+1\right)^{-1} \hat{\omega}^{-1 / 4}\right\| ; \\
\left\|\hat{k}^{j} \hat{\omega}^{-1 / 4}\left(\mathbf{x}^{2}+1\right)^{-1} \hat{\omega}^{-1 / 4} \hat{\omega}^{-1 / 4}\left(\mathbf{x}^{2}+1\right)^{-1} \hat{\omega}^{-1 / 4}\right\| ;
\end{gathered}
$$

where $\hat{k}^{j}=-i \partial / \partial x^{j}$.

To check this statement, it is sufficient to notice that lemma A.29 of Appendix A implies that the operators

$$
\left[\hat{\omega}^{\alpha} ;\left(\mathbf{x}^{2}+1\right)^{-1}\right] ; \quad\left[\hat{\omega}^{\alpha} ; x^{s}\left(\mathbf{x}^{2}+1\right)^{-1}\right] ; \quad\left[\hat{\omega}^{\alpha} ; x^{l} x^{s}\left(\mathbf{x}^{2}+1\right)^{-1}\right]
$$

are bounded if $\alpha \leq 1$.

To prove the second part of $\mathrm{P} 1$, represent it in the following form:

$$
\left\|e^{i A_{j} t} T^{1 / 2} e^{-i A_{j} t} T^{-1 / 2}\right\| \equiv\left\|T_{j}^{1 / 2}(t) T^{-1 / 2}\right\| \leq C ; \quad\left\|T_{j}(t) T^{-1}\right\| \leq C .
$$

It is necessary to investigate the Poincare transformation properties of the operators $\hat{x}^{j}$ and $\hat{k}^{j}$.

Notice that the following relations are satisfied:

$$
\begin{aligned}
& e^{i \hat{\omega} t} \hat{x}^{l} e^{-i \hat{\omega} t}=\hat{x}^{l}+\hat{k}^{l} \hat{\omega}^{-1} t, \quad e^{i \hat{\omega} t} \hat{k}^{l} e^{-i \hat{\omega} t}=\hat{k}^{l} ; \\
& e^{i \hat{k}^{s} a^{s}} \hat{x}^{l} e^{-i \hat{k}^{s} a^{s}}=\hat{x}^{l}+a^{l} ; \quad e^{i \hat{k}^{s} a^{s}} \hat{k}^{l} e^{-i \hat{k}^{s} a^{s}}=\hat{k}^{l} ; \\
& e^{\frac{i \tau}{2} \theta_{m s}\left(\hat{x}^{m} \hat{k}^{s}-\hat{x}^{s} \hat{k}^{m}\right)} \hat{x}^{l} e^{-\frac{i \tau}{2} \theta_{m s}\left(\hat{x}^{m} \hat{k}^{s}-\hat{x}^{s} \hat{k}^{m}\right)}=\left(e^{-\tau \theta} \hat{x}\right)^{l} \text {; } \\
& e^{\frac{i \tau}{2} \theta_{m s}\left(\hat{x}^{m} \hat{k}^{s}-\hat{x}^{s} \hat{k}^{m}\right)} \hat{k}^{l} e^{-\frac{i \tau}{2} \theta_{m s}\left(\hat{x}^{m} \hat{k}^{s}-\hat{x}^{s} \hat{k}^{m}\right)}=\left(e^{-\tau \theta} \hat{k}\right)^{l} ; \\
& e^{i L^{1} \tau} \hat{k}^{l} e^{-i L^{1} \tau}=\hat{k}^{l}, \quad l \geq 2 ; \quad e^{i L^{1} \tau} \hat{k}^{1} e^{-i L^{1} \tau}=\hat{k}^{1} \cosh \tau-\hat{\omega} \sinh \tau .
\end{aligned}
$$


The operators $\hat{X}^{l}(\tau)=e^{i L^{1} \tau} \hat{x}^{l} e^{-i L^{1} \tau}$ have the following Weyl symbols:

$$
X^{1}=\frac{\omega_{\mathbf{k}}}{\omega_{\mathbf{k}} \cosh \tau-k^{1} \sinh \tau} x^{1} ; \quad X^{\alpha}=x^{\alpha}+\frac{k^{\alpha} \sinh \tau x^{1}}{\omega_{\mathbf{k}} \cosh \tau-k^{1} \sinh \tau}
$$

To check the properties, it is sufficient to show that they are satisfied at $\tau=0$ and show that the derivatives of left-hand and right-hand sides of these relations coincide.

Making use of commutation relations $\left[x^{s}, f(\hat{k})\right]=i \frac{\partial f}{\partial k^{s}}(\hat{k})$ and boundedness of the operators (3.39), we find that operators $(\underline{3.40})$ are bounded uniformly with respect to $t \in\left[0, t_{1}\right]$. property $\mathrm{P} 1$ is checked.

\subsection{Choice of the operator $\mathcal{R}$}

Let us choose operator $\mathcal{R}$ in order to satisfy properties P1-P5, P7. We will use the notions of Appendix A (subsection A.5). First, we construct such an asymptotic expansion of a Weyl symbol $\underline{\mathcal{R}}_{N}$ that for $\underline{\mathcal{R}}=\underline{\mathcal{R}}_{N}$

$$
\begin{gathered}
\operatorname{deg}\left[\delta_{B}^{l} \underline{\mathcal{R}}-\underline{\mathcal{R}} * x^{l} * \underline{\mathcal{R}}-x^{l}\left(\omega_{k}^{2}+V^{\prime \prime}(\Phi(\mathbf{x}))\right)\right]>\max \{d / 2, d-1\} \\
\operatorname{deg}\left[\delta_{H} \underline{\mathcal{R}}-\underline{\mathcal{R}} * \underline{\mathcal{R}}-\left(\omega_{k}^{2}+V^{\prime \prime}(\Phi(\mathbf{x}))\right)\right]>\max \{d / 2, d-1\} .
\end{gathered}
$$

Next, we will construct another asymptotic expansion of a Weyl symbol $\underline{\mathcal{R}}$ which obeys the condition $\operatorname{Im} \underline{\mathcal{R}}>0$ and approximately equals to $\underline{\mathcal{R}}_{N}$ at large $|k|$, so that eqs.(3.41) are satisfied.

This will imply that properties P2-P5, P6' are satisfied.

Let us define the expansions $\underline{\mathcal{R}}_{N}$ with the help of the following recursive relations. Set

$$
\begin{gathered}
\underline{\mathcal{R}}_{0}=i \omega_{k} ; \\
\underline{\mathcal{S}}_{n}=-\delta_{H} \underline{\mathcal{\mathcal { R }}}_{n}+\underline{\mathcal{\mathcal { R }}}_{n} * \underline{\mathcal{\mathcal { R }}}_{n}+\omega_{k}^{2}+V^{\prime \prime}(\Phi(\mathbf{x})) ; \\
\underline{\mathcal{\mathcal { R }}}_{n+1}=\underline{\mathcal{\mathcal { R }}}_{n}+\frac{i}{2 \omega_{k}} \underline{\mathcal{S}}_{n} .
\end{gathered}
$$

Lemma 3.1. The following relation is satisfied:

$$
\operatorname{deg} \underline{\mathcal{S}}_{n}=n .
$$

Proof. For $n=0, \underline{\mathcal{S}}_{0}=V^{\prime \prime}(\Phi(\mathbf{x}))$, so that statement of lemma is satisfied. Suppose that statement of lemma is justified for $n<N$. Check it for $n=N$. One has

$$
\underline{\mathcal{S}}_{N}=\underline{\mathcal{S}}_{N-1}+\underline{\mathcal{R}}_{N} *\left(\frac{i}{2 \omega_{k}} \underline{\mathcal{S}}_{N-1}\right)+\left(\frac{i}{2 \omega_{k}} \underline{\mathcal{S}}_{N-1}\right) * \underline{\mathcal{R}}_{N}+\left(\frac{i}{2 \omega_{k}} \underline{\mathcal{S}}_{N-1}\right) *\left(\frac{i}{2 \omega_{k}} \underline{\mathcal{S}}_{N-1}\right)-\frac{i}{2 \omega_{k}} \delta_{H} \underline{\mathcal{S}}_{N-1}
$$

Since

$$
\operatorname{deg}\left[\left(\frac{i}{2 \omega_{k}} \underline{\mathcal{S}}_{N-1}\right) *\left(\frac{i}{2 \omega_{k}} \underline{\mathcal{S}}_{N-1}\right)-\frac{i}{2 \omega_{k}} \delta_{H} \underline{\mathcal{S}}_{N-1}\right] \geq \operatorname{deg} \underline{\mathcal{S}}_{N-1}+1=N
$$

and

$$
\begin{gathered}
\underline{\mathcal{S}}_{N}=\underline{\mathcal{S}}_{N-1}+\underline{\mathcal{R}}_{N} *\left(\frac{i}{2 \omega_{k}} \underline{\mathcal{S}}_{N-1}\right)+\left(\frac{i}{2 \omega_{k}} \underline{\mathcal{S}}_{N-1}\right) * \underline{\mathcal{R}}_{N} \simeq \\
\underline{\mathcal{S}}_{N-1}+\underline{\mathcal{R}}_{N}\left(\frac{i}{2 \omega_{k}} \underline{\mathcal{S}}_{N-1}\right)+\left(\frac{i}{2 \omega_{k}} \underline{\mathcal{S}}_{N-1}\right) \underline{\mathcal{R}}_{N}=0
\end{gathered}
$$

up to terms of the degree $N$, one finds

$$
\operatorname{deg} \underline{\mathcal{S}}_{N}=N
$$

Lemma 3.1 is proved.

Denote

$$
\underline{X}_{n}^{l}=-\delta_{B}^{l} \underline{\mathcal{R}}_{n}+\underline{\mathcal{R}}_{n} * x^{l} * \underline{\mathcal{R}}_{n}+x^{l}\left(\omega_{k}^{2}+V^{\prime \prime}(\Phi(\mathbf{x}))\right) .
$$

Lemma 3.2. The following property is obeyed:

$$
\delta_{B}^{l} \underline{\mathcal{S}}_{n}-\delta_{H} \underline{X}_{n}^{l}=-\underline{X}_{n}^{l} * \underline{\mathcal{R}}_{n}-\underline{\mathcal{R}}_{n} * \underline{X}_{n}^{l}+\underline{\mathcal{S}}_{n} * x^{l} * \underline{\mathcal{R}}_{n}+\underline{\mathcal{R}}_{n} * x^{l} * \underline{\mathcal{S}}_{n} .
$$


Proof. Denote

$$
\underline{F}_{n}^{l}=\delta_{B}^{l} \underline{\mathcal{S}}_{n}-\delta_{H} \underline{X}_{n}^{l}+\underline{X}_{n}^{l} * \underline{\mathcal{R}}_{n}+\underline{\mathcal{R}}_{n} * \underline{X}_{n}^{l}-\underline{\mathcal{S}}_{n} * x^{l} * \underline{\mathcal{R}}_{n}-\underline{\mathcal{R}}_{n} * x^{l} * \underline{\mathcal{S}}_{n}
$$

One has

$$
\begin{gathered}
\underline{F}_{n}^{l}=\left(\delta_{B}^{l}-x^{l} \delta_{H}\right) V^{\prime \prime}(\Phi(\mathbf{x}))+\left[\delta_{H} ; \delta_{B}^{l}\right] \underline{\mathcal{R}}_{n}-\left[x ^ { l } \left(\omega_{k}^{2}+\right.\right. \\
V^{\prime \prime}(\Phi(\mathbf{x}))-\left(\omega_{k}^{2}+V^{\prime \prime}(\Phi(\mathbf{x})) * x^{l}\right] * \underline{\mathcal{R}}_{n}+\underline{\mathcal{R}}_{n} *\left[x ^ { l } \left(\omega_{k}^{2}+V^{\prime \prime}(\Phi(\mathbf{x}))-x^{l} *\left(\omega_{k}^{2}+V^{\prime \prime}(\Phi(\mathbf{x}))\right]\right.\right.
\end{gathered}
$$

It follows from the definition of the Weyl symbol that

$$
x^{l} * f(x, k)=\left(x^{l}+\frac{i}{2} \frac{\partial}{\partial k^{l}}\right) f(x, k)
$$

One also has

$$
\left(\delta_{B}^{l}-x^{l} \delta_{H}\right) V^{\prime \prime}(\Phi(\mathbf{x}))=0
$$

Thus,

$$
\underline{F}_{n}^{l}=\left[\delta_{H} ; \delta_{B}^{l}\right] \underline{\mathcal{R}}_{n}+i k^{l} * \underline{\mathcal{R}}_{n}-\underline{\mathcal{R}}_{n} * i k^{l}=\frac{\partial \underline{\mathcal{R}}_{n}}{\partial x^{l}}-\delta_{P}^{l} \underline{\mathcal{R}}_{n} .
$$

However, the property

$$
\frac{\partial \underline{\mathcal{R}}_{n}}{\partial x^{l}}=\delta_{P}^{l} \underline{\mathcal{R}}_{n}
$$

which means that eq. (3.21) is satisfied is checked by induction. Lemma 3.2 is proved.

Lemma 3.3. The following properties are satisfied:

1. $\operatorname{deg} \underline{X}_{n}^{l}=n$.

2. $\operatorname{deg}\left(\underline{X}_{n}^{l}-x^{l} \underline{\mathcal{S}}_{n}\right) \geq n+1$.

Proof. It follows from the results of Appendix A that $\underline{X}_{n}^{l}$ is an asymptotic expansion of a Weyl symbol. Let $\operatorname{deg} \underline{X}_{n}^{l}=\alpha$.

Suppose that $\alpha<n$. Then the left-hand side of eqs. (3.43) is of the degree $\alpha$, the degree of the righthand side of eq.(3.43) is greater than or equal to $\alpha-1$. In the leading order in $1 /|k|$ the right-hand side has the form one has $\left(-2 i \omega_{k} \underline{X}_{n}^{l}\right)$ and its degree should be greater than or equal to $\alpha$. Therefore, $\operatorname{deg} \underline{X}_{n}^{l} \geq \alpha+1$. We obtain a contradiction.

Suppose $\alpha>n$. Then the left-hand side of eq.(3.43) is of the degree $n$, the right-hand side in the leading order in $1 /|k|$ has the form $2 i \omega_{k} x^{l} \underline{\mathcal{S}}_{n}$. so that $\operatorname{deg} \underline{\mathcal{S}}_{n}$ should obey the inequality $\operatorname{deg} \underline{\mathcal{S}}_{n} \geq n+1$. We also obtain a contradiction.

Thus, $\alpha=n$. In the leading order in $1 /|k|$ one has

$$
0 \simeq-2 i \omega_{k}\left(\underline{X}_{n}^{l}-x^{l} \underline{\mathcal{S}}_{n}\right)
$$

up to terms of the degree $n$, so that $\operatorname{deg}\left(\underline{X}_{n}^{l}-x^{l} \underline{\mathcal{S}}_{n}\right) \geq n+1$. Lemma 3.3 is proved.

We see that for $N \geq \max \{d / 2, d-1\}$ the properties (3.41) are satisfied.

Lemma 3.4. Let $\underline{\mathcal{R}}^{(1)}$ and $\underline{\mathcal{R}}^{(2)}$ be asymptotic expansions of Weyl symbols, deg $\underline{\mathcal{R}}^{(1)}=\operatorname{deg} \underline{\mathcal{R}}^{(2)}=-1$ and $\operatorname{deg}\left(\underline{\mathcal{R}}^{(1)}-\underline{\mathcal{R}}^{(2)}\right)=N+1$. Then

$$
\operatorname{deg}\left(\underline{X}^{(1) l}-\underline{X}^{(2) l}\right)=N
$$

and

$$
\operatorname{deg}\left(\underline{\mathcal{S}}^{(1)}-\underline{\mathcal{S}}^{(2)}\right)=N
$$

Proof. Denote $\underline{\mathcal{R}}^{(1)}-\underline{\mathcal{R}}^{(2)}=\underline{D}$. Then

$$
\underline{X}^{(1) l}-\underline{X}^{(2) l}=-\delta_{B}^{l} \underline{D}+\underline{\mathcal{R}}^{(1)} * x^{l} * \underline{D}+\underline{D} * x^{l} * \underline{\mathcal{R}}^{(1)}+\underline{D} * \underline{D} * x^{l} * \underline{D} .
$$


We see that $\operatorname{deg}\left(\underline{X}^{(1) l}-\underline{X}^{(2) l}\right)=N$. The second statement is checked analogously. Lemma 3.4 is proved.

Let us construct such an asymptotic expansion $\underline{\mathcal{R}}$ that $\operatorname{deg}\left(\underline{\mathcal{R}}-\underline{\mathcal{R}}_{N}\right)=N+1$ and $\operatorname{Im} \underline{\mathcal{R}}>0$. We will look for $\underline{\mathcal{R}}$ as follows (cf. [36]),

$$
\underline{\mathcal{R}}=\underline{\mathcal{A}}+i \omega_{k}^{1 / 4} * \exp \underline{\mathcal{B}} * \omega_{k}^{1 / 4} * \exp \underline{\mathcal{B}} * \omega_{k}^{1 / 4}
$$

where $\underline{\mathcal{A}}$ and $\underline{\mathcal{B}}$ are real asymptotic expansions. Then

$$
\begin{gathered}
\underline{\Gamma^{1 / 2}}=\omega_{k}^{1 / 4} * \exp \underline{\mathcal{B}} * \omega_{k}^{1 / 4} ; \\
\underline{\Gamma^{-1 / 2}}=\omega_{k}^{-1 / 4} * \exp (-\underline{\mathcal{B}}) * \omega_{k}^{-1 / 4}
\end{gathered}
$$

are also asymptotic expansions of Weyl symbols. Choose $\underline{\mathcal{A}}$ and $\underline{\mathcal{B}}$ to be polynomials,

$$
\underline{\mathcal{A}}=\sum_{s=1}^{S_{1}} \frac{A_{s}\left(\mathbf{x}, \mathbf{k} / \omega_{\mathbf{k}}\right)}{\omega_{\mathbf{k}}^{2 s}}, \quad \underline{\mathcal{B}}=\sum_{s=1}^{S_{2}} \frac{B_{s}\left(\mathbf{x}, \mathbf{k} / \omega_{\mathbf{k}}\right)}{\omega_{\mathbf{k}}^{2 s}},
$$

where $S_{1}=[N / 2], S_{2}=\left[\frac{N+1}{2}\right]$.

Lemma 3.5. There exists unique functions $A_{1}, \ldots, A_{S_{1}}, B_{1}, \ldots, B_{S_{2}}$ such that deg $\left(\underline{\mathcal{R}}-\underline{\mathcal{R}}_{N}\right)=N+1$.

Proof. It follows from recursive relations (3.42) that

$$
\begin{gathered}
\operatorname{Re} \underline{\mathcal{R}}_{N}=\sum_{s=1}^{\infty} \frac{A_{N, s}\left(\mathbf{x}, \mathbf{k} / \omega_{\mathbf{k}}\right)}{\omega_{\mathbf{k}}^{2 s}}, \\
\operatorname{Im} \underline{\mathcal{R}}_{N}=\omega_{\mathbf{k}}+\sum_{s=1}^{\infty} \frac{C_{N, s}\left(\mathbf{x}, \mathbf{k} / \omega_{\mathbf{k}}\right)}{\omega_{\mathbf{k}}^{2 s}} .
\end{gathered}
$$

Therefore, $A_{s}=A_{N, s}$, so that $\underline{\mathcal{A}}$ is uniquely defined. Denote

$$
\underline{\mathcal{B}_{s}}=\frac{B_{s}\left(\mathbf{x}, \mathbf{k} / \omega_{\mathbf{k}}\right)}{\omega_{\mathbf{k}}^{2 s}}
$$

Show that $\underline{\mathcal{B}_{s}}$ is uniquely defined. In the leading order in $1 /|\mathbf{k}|$, one has

$$
\operatorname{Im} \underline{\mathcal{R}} \simeq \omega_{\mathbf{k}}+2 \underline{\mathcal{B}_{1}} \omega_{\mathbf{k}}
$$

so that $B_{1}=C_{N, 1} / 2$. Suppose that one can choose $\underline{\mathcal{B}_{1}}, \ldots, \underline{\mathcal{B}_{s-1}}$ in such a way that the degree of the asymptotic expansion of a Weyl symbol

$$
\underline{F}_{N, s}=\operatorname{Im} \underline{\mathcal{R}}_{N}-\omega_{\mathbf{k}}^{1 / 4} * \exp \left(\underline{\mathcal{B}}_{1}+\ldots+\underline{\mathcal{B}}_{s-1}\right) * \omega_{\mathbf{k}}^{1 / 2} * \exp \left(\underline{\mathcal{B}}_{1}+\ldots+\underline{\mathcal{B}}_{s-1}\right) * \omega_{\mathbf{k}}^{1 / 4}
$$

satisfies the inequality

$$
\operatorname{deg} \underline{F}_{N, s} \geq 2 s-1 \text {. }
$$

Choose $\underline{\mathcal{B}}_{s}$ in such a way that $\operatorname{deg} \underline{F}_{N, s} \geq 2 s-1$. One has

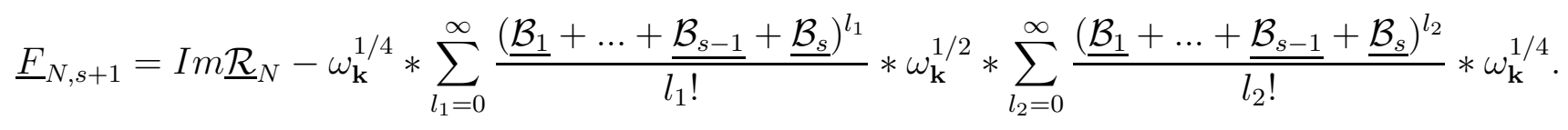

Up to terms of the degree $2 s+1$, one has

$$
\begin{gathered}
\underline{F}_{N, s+1} \simeq \operatorname{Im} \underline{\mathcal{R}}_{N}-\omega_{\mathbf{k}}^{1 / 4} *\left(\sum_{l_{1}=0}^{\infty} \frac{\underline{\left(\mathcal{B}_{1}+\ldots+\underline{\mathcal{B}}_{s-1}\right)^{l_{1}}}}{l_{1} !}+\underline{\mathcal{B}}_{s}\right) * \omega_{\mathbf{k}}^{1 / 2} *\left(\sum_{l_{2}=0}^{\infty} \frac{\underline{\left(\mathcal{B}_{1}+\ldots+\mathcal{B}_{s-1}\right)^{l_{2}}}}{l_{2} !}+\underline{\mathcal{F}_{s}}\right) * \omega_{\mathbf{k}}^{1 / 4}-\underline{2 \underline{\mathcal{B}_{s}}} \omega_{\mathbf{k}} .
\end{gathered}
$$

Since

$$
\underline{F}_{N, s}=\frac{1}{\omega_{\mathbf{k}}^{2 s-1}} \sum_{l=0}^{\infty} \frac{F_{N, s, l}\left(\mathbf{x}, \mathbf{k} / \omega_{\mathbf{k}}\right)}{\omega_{\mathbf{k}}^{l}}
$$


one finds that

$$
\underline{\mathcal{B}_{s}}=\frac{1}{2 \omega_{k}^{2 s}} F_{N, s, 0}\left(\mathbf{x}, \mathbf{k} / \omega_{\mathbf{k}}\right)
$$

is uniquely defined. Lemma 3.5 is proved.

Thus, we have constructed the operator $\mathcal{R}$ such that properties (3.41) are satisfied. We obtain the following theorem.

Theorem 3.6. Properties (3.21), P2-P5, P6'(a) are satisfied.

This theorem is a direct corollary of the results of Appendix A. Property P1 is satisfied because of construction of the operator $\mathcal{R}$. Properties P2-P5, P6'(a) are corollaries of Theorems A.31, A.32, A.33, properties (3.41) and lemmas A.8, A.9, A.19.

\subsection{Regularization and renormalization of a trace}

The purpose of this subsection is to specify functionals $\operatorname{Tr}_{R} \Gamma$ and $\operatorname{Tr}_{R} x^{k} \Gamma$ of arguments $\Phi, \Pi$ in order to satisfy properties P6'(b), (3.38). We want the renormalized trace to satisfy properties like these:

(i) $\operatorname{Tr}_{R} \hat{A}=\operatorname{Tr} \hat{A}$ if $\hat{A}$ is of the trace class;

(ii) $\operatorname{Tr}_{R}(\hat{A}+\lambda \hat{B})=\operatorname{Tr}_{R} \hat{A}+\lambda \operatorname{Tr}_{R} \hat{B}$;

(iii) $\operatorname{Tr}_{R}[\hat{A} ; \hat{B}]=0$;

(iv) $\operatorname{Tr}_{R} \hat{A}_{n} \rightarrow 0$ if $A_{n} \rightarrow 0$

for such class of operators that is as wide as possible. Under these conditions, properties P6'(b) and (3.38) are satisfied. However, one cannot specify such a renormalized trace. Namely, one should have

$$
\operatorname{Tr}_{R}\left[\hat{x}_{j} ; \mathcal{W}\left(\frac{k_{j}}{\omega_{\mathbf{k}}^{l}} f(\mathbf{x})\right)\right]=0
$$

where $f \in S\left(\mathbf{R}^{d}\right)$. $\mathcal{W}(A)$ is a Weyl quantization of the function $A$ (see appendix A). Property (3.44) means that

$$
\operatorname{Tr}_{R} \mathcal{W}\left(i \frac{\partial}{\partial k_{i}} \frac{k_{j}}{\omega_{\mathbf{k}}^{l}} f(\mathbf{x})\right)=0
$$

Therefore,

$$
\delta_{i j} \operatorname{Tr}_{R} \mathcal{W}\left(\frac{f(\mathbf{x})}{\omega_{\mathbf{k}}^{l}}\right)-l \operatorname{Tr}_{R} \mathcal{W}\left(\frac{k_{i} k_{j}}{\omega_{\mathbf{k}}^{l+2}} f(\mathbf{x})\right)=0
$$

Choose $l=d$. Consider $i=j$ in eq.(3.45) and perform the summation over $i$. Making use of the relation $\omega_{\mathbf{k}}^{2}-k_{i} k_{i}=m^{2}$, we find

$$
\operatorname{Tr}_{R} \mathcal{W}\left(m^{2} \omega_{\mathbf{k}}^{-d-2} f(\mathbf{x})\right)=0
$$

However, the operator with Weyl symbol $m^{2} f(\mathbf{x}) \omega_{\mathbf{k}}^{-d-2}$ is of the trace class. Its trace is nonzero, provided that $\int d \mathbf{x} f(x) \neq 0$.

However, we can introduce a notion of a trace for asymptotic expansions of Weyl symbols. The trace will be specified not only by operator but also by its asymptotic expansion which is not unique (see remark after definition A.6).

Let $\underline{A}=(A, \check{A})$ be asymptotic expansion of a Weyl symbol. Suppose that the coefficients $A_{l}$ of the formal asymptotic expansion

$$
\check{A} \equiv \sum_{l=0}^{\infty} \omega_{\mathbf{k}}^{-\alpha-l} A_{l}\left(x, \mathbf{k} / \omega_{\mathbf{k}}\right)
$$

are polynomial in $\mathbf{k} / \omega_{\mathbf{k}}$. One formally has

$$
\operatorname{Tr}_{R} \underline{A}=\sum_{l=0}^{l_{0}} \int \frac{d \mathbf{k} d \mathbf{x}}{(2 \pi)^{d}} \frac{1}{\omega_{\mathbf{k}}^{\alpha+l}} A_{l}\left(\mathbf{x}, \mathbf{k} / \omega_{\mathbf{k}}\right)+\int \frac{d \mathbf{k} d \mathbf{x}}{(2 \pi)^{d}}\left(A(\mathbf{x}, \mathbf{k})-\sum_{l=0}^{l_{0}} \frac{1}{\omega_{\mathbf{k}}^{\alpha+l}} A_{l}\left(\mathbf{x}, \mathbf{k} / \omega_{\mathbf{k}}\right)\right) .
$$


For $\alpha+l_{0}+1>d$, the last integral in the right-hand side of eq.(3.46) converges. To specify trace, it is sufficient then to specify values of integrals

$$
I_{i_{1} \ldots i_{n}}^{s, n}=\int \frac{d \mathbf{k}}{\omega_{\mathbf{k}}^{s}} \frac{k_{i_{1}}}{\omega_{\mathbf{k}}} \ldots \frac{k_{i_{n}}}{\omega_{\mathbf{k}}}
$$

for $s \leq d$ which are divergent. We will define the quantities (3.47), making use of the following argumentation.

1. We are going to specify to specify trace in such a way that

$$
\operatorname{Tr}_{R} \frac{\partial}{\partial k_{i}} \underline{A}=0
$$

Let

$$
\underline{A}=\frac{1}{\omega_{\mathbf{k}}^{s-1}} \frac{k_{j_{1}}}{\omega_{\mathrm{k}}} \ldots \frac{k_{j_{n+1}}}{\omega_{\mathrm{k}}}
$$

property (3.48) implies the following recursive relations

$$
\sum_{s=1}^{n+1} \delta_{i j_{s}} I_{j_{1} \ldots j_{s-1} j_{s+1} \ldots j_{n+1}}^{s, n}=(s+n) I_{i j_{1} \ldots j_{n+1}}^{s, n+2} .
$$

Therefore, $I^{s, n}=0$ for odd $n$, while for even $n I^{s, n}$ is defined from eqs. (3.49), for example, $I_{i j}^{s, 2}=\frac{1}{s} \delta_{i j} I^{s, 0}$. Therefore, it is sufficient to define integrals

$$
I^{s, 0}=\int d \mathbf{k} \omega_{\mathbf{k}}^{-s}
$$

Let us use the approach based on the dimensional regularization 49, 50. It is based on considering integrals (3.50) at arbitrary dimensionality of space-time. Expression (3.50) appears to be a meromorphic function of $d$. Substracting the poles corresponding to sufficiently small positive integer values of $d$, we obtain a finite expression.

Formally, one has

$$
I^{s, 0}=\frac{1}{\Gamma(s / 2)} \int_{0}^{\infty} d \alpha \alpha^{s / 2-1} \int d \mathbf{k} e^{-\alpha\left(\mathbf{k}^{2}+m^{2}\right)}=\frac{\pi^{d / 2}}{\Gamma(s / 2)} \frac{\Gamma\left(\frac{s-d}{2}\right)}{m^{s-d}} .
$$

If $\frac{s-d}{2}=-N$ is a nonpositive integer number, one should modify the definition of $I^{s, 0}$. Change $d \rightarrow d-2 \varepsilon$. One finds:

$$
\begin{gathered}
I^{s, 0}=\frac{\pi^{d / 2}}{\Gamma(s / 2) m^{s-d}} \frac{\Gamma(1+\varepsilon)\left(\pi m^{2}\right)^{-\varepsilon}}{(-N+\varepsilon) \ldots(-1+\varepsilon) \varepsilon} \\
\simeq \frac{\pi^{d / 2}(-1)^{N}}{\Gamma(s / 2) m^{s-d} N ! \varepsilon}\left(1+\varepsilon\left(-\ln \left(\pi m^{2}\right)+\Gamma^{\prime}(1)+1+\ldots+N^{-1}\right)\right)+O(\varepsilon) .
\end{gathered}
$$

In the $M S$ renormalization scheme [50], one should omit the term $O\left(\varepsilon^{-1}\right)$. There is also an $\overline{M S}$ renormalization scheme in which one omits also a fixed term of order $O(1)$. Let us omit the term $-\ln \left(\pi m^{2}\right)+\Gamma^{\prime}(1)$. We obtain the following renormalized value of the integral:

$$
I_{r e n}^{s, 0}=\frac{\pi^{d / 2}}{\Gamma(s / 2) m^{s-d}} \frac{(-1)^{N}}{N !}(1+\ldots+1 / N),
$$

provided that $N=\frac{d-s}{2}$ is a nonnegative integer number.

Therefore, we have defined the renormalized trace of an asymptotic expansion of a Weyl symbol by formaula (3.46), provided that the coefficient functions are polynomials in $\mathbf{k} / \omega_{\mathbf{k}}$.

Let us investigate properties of the renormalized trace. Some properties are direct corollaries of definition (3.46).

Lemma 3.7. The following properties are satisfied:

(i) $\operatorname{Tr}_{R}(\underline{A}+\lambda \underline{B})=0$; 
(ii) $\operatorname{Tr}_{R} \frac{\partial \underline{A}}{\partial k_{i}}=0 ; \operatorname{Tr}_{R} \frac{\partial \underline{A}}{\partial x_{i}}=0$;

(ii) Let $E-\lim _{n \rightarrow \infty} \underline{A}_{n}=\underline{A}$. Then $\lim _{n \rightarrow \infty} \operatorname{Tr}_{R} \underline{A}_{n}=\operatorname{Tr}_{R} \underline{A}$.

(iv) Let $\operatorname{deg} \underline{A}>d$. Then $\operatorname{Tr}_{R} \underline{A}=\operatorname{Tr} A$.

Corollary. The property AP9 is satisfied.

Let us check that $\operatorname{Tr}_{R}(\underline{A} * \underline{B}-\underline{B} * \underline{A})=0$. First of all, prove the following statement.

Lemma 3.8. $\operatorname{Tr}_{R} \underline{A} * \underline{B}=\operatorname{Tr}_{R} \underline{A B}$.

Proof. Making use of eq.(A.4), we find

$$
\begin{gathered}
(A * B)(\mathbf{x}, \mathbf{k})-(A B)(\mathbf{x}, \mathbf{k})= \\
\int \frac{d \mathbf{p}_{1} d \mathbf{p}_{2} d \mathbf{y}_{1} d \mathbf{y}_{2}}{(2 \pi)^{2 d}} \int_{0}^{1} d \alpha \frac{\partial}{\partial \alpha}\left[A\left(\mathbf{x}+\mathbf{y}_{1} ; \mathbf{k}+\alpha \frac{\mathbf{p}_{2}}{2}\right) B\left(\mathbf{x}+\mathbf{y}_{2}, \mathbf{k}_{2}-\alpha \frac{\mathbf{p}_{1}}{2}\right)\right] e^{-i \mathbf{p}_{1} \mathbf{y}_{1}-i \mathbf{p}_{2} \mathbf{y}_{2}}= \\
-\frac{i}{2} \int \frac{d \mathbf{p}_{1} d \mathbf{p}_{2} d \mathbf{y}_{1} d \mathbf{y}_{2}}{(2 \pi)^{2 d}} \int_{0}^{1} d \alpha\left[\frac{\partial}{\partial k^{i}} A\left(\mathbf{x}+\mathbf{y}_{1} ; \mathbf{k}+\alpha \frac{\mathbf{p}_{2}}{2}\right) \frac{\partial}{\partial x^{i}} B\left(\mathbf{x}+\mathbf{y}_{2}, \mathbf{k}_{2}-\alpha \frac{\mathbf{p}_{1}}{2}\right)-\right. \\
\left.\frac{\partial}{\partial x^{i}} A\left(\mathbf{x}+\mathbf{y}_{1} ; \mathbf{k}+\alpha \frac{\mathbf{p}_{2}}{2}\right) \frac{\partial}{\partial k^{i}} B\left(\mathbf{x}+\mathbf{y}_{2}, \mathbf{k}_{2}-\alpha \frac{\mathbf{p}_{1}}{2}\right)\right] e^{-i \mathbf{p}_{1} \mathbf{y}_{1}-i \mathbf{p}_{2} \mathbf{y}_{2}}=-\frac{i}{2} \frac{\partial C^{i}(\mathbf{x}, \mathbf{k})}{\partial k^{i}}
\end{gathered}
$$

with

$$
\begin{gathered}
C^{i}(\mathbf{x}, \mathbf{k})=\int \frac{d \mathbf{p}_{1} d \mathbf{p}_{2} d \mathbf{y}_{1} d \mathbf{y}_{2}}{(2 \pi)^{2 d}} \int_{0}^{1} d \alpha\left[A\left(\mathbf{x}+\mathbf{y}_{1} ; \mathbf{k}+\alpha \frac{\mathbf{p}_{2}}{2}\right) \frac{\partial}{\partial x^{i}} B\left(\mathbf{x}+\mathbf{y}_{2}, \mathbf{k}_{2}-\alpha \frac{\mathbf{p}_{1}}{2}\right)-\right. \\
\left.B\left(\mathbf{x}+\mathbf{y}_{2}, \mathbf{k}_{2}-\alpha \frac{\partial}{\partial x^{i}} A\left(\mathbf{x}+\mathbf{y}_{1} ; \mathbf{k}+\alpha \frac{\mathbf{p}_{2}}{2}\right) \frac{\mathbf{p}_{1}}{2}\right)\right] e^{-i \mathbf{p}_{1} \mathbf{y}_{1}-i \mathbf{p}_{2} \mathbf{y}_{2}} .
\end{gathered}
$$

One also has

$$
\check{A} * \check{B}-\check{A} \check{B}=-\frac{i}{2} \frac{\partial \check{C}^{j}}{\partial k^{j}}
$$

with

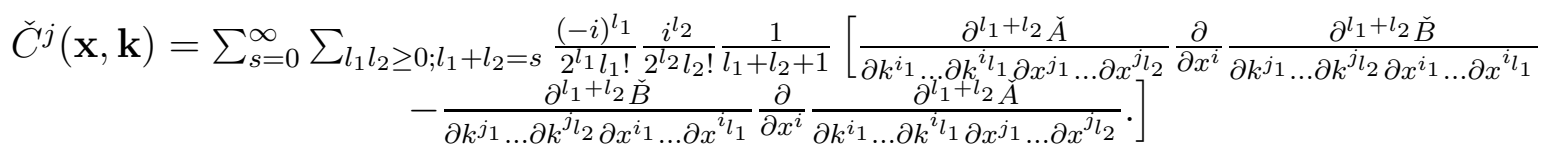

Analogously to Appendix $\mathrm{C}$, one finds that $\left(C^{j}, \check{C}^{j}\right) \equiv \underline{C^{j}}$ is an asymptotic expansion of a Weyl symbol. It follows from lemma 3.7 that $\operatorname{Tr}_{R} \frac{\partial \underline{C}^{j}}{\partial k^{j}}=0$. We obtain statement of lemma 3.8.

Lemma 3.9. For $\operatorname{deg} \underline{B} \geq 2, \operatorname{Tr}_{R} x^{k} \omega_{\mathbf{k}} * \underline{B}=\operatorname{Tr}_{R} x^{k} \omega_{\mathbf{k}} \underline{B}$ and $\operatorname{Tr}_{R} \omega_{\mathbf{k}} * \underline{B}=\operatorname{Tr}_{R} \omega_{\mathbf{k}} \underline{B}$.

The proof is analogous.

Corollary 1. The following relations are satisfied:

1. $\operatorname{Tr}_{R}(\underline{A} * \underline{B}-\underline{B} * \underline{A})=0$;

2. $\operatorname{Tr}_{R}\left(x^{k} \omega_{\mathbf{k}} * \underline{B}-\underline{B} * x^{k} \omega_{\mathbf{k}}\right)=0$;

3. $\operatorname{Tr}_{R}\left(\omega_{\mathbf{k}} * \underline{B}-\underline{B} * \omega_{\mathbf{k}}\right)=0$.

Corollary 2. Property (3.38) is satisfied.

Thus, we have constructed functionals $\operatorname{Tr}_{R} x^{k} \hat{\Gamma} \equiv \operatorname{Tr}_{R} x^{k} \underline{\Gamma}$ and $\operatorname{Tr}_{R} \hat{\Gamma} \equiv \operatorname{Tr}_{R} \underline{\Gamma}$ such that properties (3.38) and P6'(b) are satisfied.

Note that the "finite renormalization" [25] can be also be made. One can add quantities $\Delta \operatorname{Tr}_{R} x^{k} \hat{\Gamma}$ and $\Delta \operatorname{Tr}_{R} \hat{\Gamma}$ to renormalized traces in such a way that

$$
\begin{gathered}
\delta_{k}^{P} \Delta \operatorname{Tr}_{R} \hat{\Gamma}=0 ; \quad \delta_{k l}^{M} \Delta \operatorname{Tr}_{R} \hat{\Gamma}=0 ; \\
\delta_{l}^{P} \Delta \operatorname{Tr}_{R} x^{k} \hat{\Gamma}=-\delta^{k l} \Delta \operatorname{Tr}_{R} \hat{\Gamma} ; \quad \delta_{k l}^{M} \Delta \operatorname{Tr}_{R} x^{k} \hat{\Gamma}=\delta^{k l} \Delta \operatorname{Tr}_{R} x^{m} \hat{\Gamma}-\delta^{m k} \Delta \operatorname{Tr}_{R} x^{l} \hat{\Gamma} \\
\delta_{l}^{B} \Delta \operatorname{Tr}_{R} x^{k} \hat{\Gamma}-\delta_{k}^{B} \Delta \operatorname{Tr}_{R} x^{l} \hat{\Gamma}=0 ; \\
\delta_{l}^{B} \Delta \operatorname{Tr}_{R} \hat{\Gamma}-\delta^{H} \Delta \operatorname{Tr}_{R} x^{l} \hat{\Gamma}=0 .
\end{gathered}
$$

This corresponds to the possibility of adding the finite one-loop counterterm to the Lagrangian.

\section{Semiclassical field}

An important feature of QFT is a notion of field. In this section we introduce the notion of a semiclassical field and check its Poincare invariance. 


\subsection{Definition of semiclassical field}

First of all, introduce the notion of a semiclassical field $\tilde{\phi}(\mathbf{x}, t: X)$ in the functional Schrodinger representation. At $t=0$, this is the operator of multiplication by $\phi(\mathbf{x})$. For arbitrary $t$, one has

$$
\tilde{\phi}(\mathbf{x}, t: X)=\tilde{U}_{-t}\left(X \leftarrow u_{t} X\right) \phi(\mathbf{x}) \tilde{U}_{t}\left(u_{t} X \leftarrow X\right),
$$

where $\tilde{U}_{t}\left(u_{t} X \leftarrow X\right)$ is the operator transforming the initial condition for the Cauchy problem for eq.(1.6) to the solution to the Cauchy problem.

The field operator in the Fock representation is related with $\tilde{\phi}$ by the transformation (2.1),

$$
\hat{\phi}(\mathbf{x}, t: X)=V_{X}^{-1} \tilde{\phi}(\mathbf{x}, t: X) V_{X} .
$$

Making use of eq.(3.15), one finds

$$
\hat{\phi}(\mathbf{x}, t: X)=\left(U_{H}^{t}(X)\right)^{-1} \hat{\phi}\left(\mathbf{x}, u_{t} X\right) U_{H}^{t}(X)
$$

Here $\hat{\phi}(\mathbf{x}: X)=i\left(\Gamma^{-1 / 2}\left(A^{+}-A^{-}\right)\right)(\mathbf{x})$, while

$$
U_{H}^{t}(X) \equiv U_{a, \Lambda}\left(u_{a, \Lambda} X \leftarrow X\right), \quad \Lambda=1, \mathbf{a}=0, a^{0}=-t .
$$

Let us define $\hat{\phi}$ mathematically as an operator distribution.

Let $S\left(\mathbf{R}^{d}\right)$ be a space of complex smooth functions $u: \mathbf{R}^{d} \rightarrow \mathbf{C}$ such that

$$
\|u\|_{l, m}=\max _{\alpha_{1}+\ldots+\alpha_{d} \leq l} \sup _{\mathbf{x} \in \mathbf{R}^{d}}(1+|\mathbf{x}|)^{m}\left|\frac{\partial^{\alpha_{1}+\ldots+\alpha_{d}}}{\partial x^{\alpha_{1}} \ldots \partial x^{\alpha_{d}}} u(\mathbf{x})\right| \rightarrow_{k \rightarrow \infty} 0 .
$$

We say that the sequence $\left\{u_{k}\right\} \in S\left(\mathbf{R}^{n}\right), k=\overline{1, \infty}$ tends to zero if $\left\|u_{k}\right\|_{l, m} \rightarrow_{k \rightarrow \infty} 0$ for all $l, m$.

Denote $\mathcal{D}=\left\{\Psi \in \mathcal{F}\left\|A^{+} T A^{-} \Psi\right\|<\infty\right\}$ (cf. [48]).

Definition 4.1. (cf. 20]). 1. An operator distribution $\phi$ defined on $\mathcal{D} \in \mathcal{F}$ is a linear mapping taking functions $f \in S\left(\mathbf{R}^{d}\right)$ to the linear operator $\phi[f]: \mathcal{D} \rightarrow \mathcal{F}$,

$$
\phi: f \in S\left(\mathbf{R}^{d}\right) \mapsto \phi[f]: \mathcal{D} \rightarrow \mathcal{F}
$$

such that $\left\|\phi\left[f_{n}\right] \Phi\right\| \rightarrow_{n \rightarrow \infty} 0$ if $f_{n} \rightarrow_{n \rightarrow \infty} 0$.

2. A sequence of operator distributions $\phi_{n}$ is called convergent to the operator distribtion $\phi$ if

$$
\left\|\phi_{n}[f] \Phi-\phi[f] \Phi\right\| \rightarrow_{n \rightarrow \infty} 0
$$

for all $\Phi \in \mathcal{D}, f \in S\left(\mathbf{R}^{d}\right)$.

We will write

$$
\phi[f] \equiv \int d \mathbf{x} \phi(\mathbf{x}) f(\mathbf{x}), \quad \mathbf{x} \in \mathbf{R}^{d} .
$$

Consider the mapping $f \mapsto \phi_{t}\{f\}, f \in S\left(\mathbf{R}^{d}\right)$ of the form

$$
\phi_{t}\{f: X\}=\int d \mathbf{x} \hat{\phi}(\mathbf{x}, t: X) f(\mathbf{x}) .
$$

It follows from the results of 48 that $\phi_{t}$ is an operator distribution being continous with respect to $t$.

Consider the mapping $f \mapsto \phi[f], f \in S\left(\mathbf{R}^{d+1}\right)$ of the form

$$
\phi[f: X]=\int d t \phi_{t}\{f(\cdot, t): X\} .
$$

Analogously, note also that $\phi$ is an operator distribution. 


\subsection{Poincare invariance of the semiclassical field}

\subsubsection{Algebraic properties}

To check the property of Poincare invariance, notice that it is sufficient to check it for partial cases: spatial translations, rotations, evolution, boost, since any Poincare transformation can be presented as a composition of these transformations. Let $g_{B}(\tau)=(a(\tau), \Lambda(\tau))$ be a one-parametric subgroup of Poincare group corresponding to the element $B$ of the Poincare algebra. The Poincare invariance property can be rewritten as

$$
\hat{\phi}[f: X]=\left(U_{B}^{\tau}(X)\right)^{+} \phi\left[v_{g_{B}(\tau)} f: U_{g_{B}(\tau)} X\right] U_{B}^{\tau}[X],
$$

where

$$
\left(v_{g_{B}(\tau)} f\right)(x)=f\left(\Lambda^{-1}(\tau)(x-a(\tau))\right) .
$$

Obviously, $v_{g_{1}} v_{g_{2}}=v_{g_{1} g_{2}}$.

Let us check relation (4.2). It is convenient to reduce the group property to an algebraic property. The formal derivative with respect to $\tau$ of the right-hand side of eq.(4.2) is

$$
\left(U_{B}^{\tau}(X)\right)^{+}\left\{i\left[H\left(B: u_{g_{B}(\tau)} X\right) ; \phi\left[v_{g_{B}(\tau)} f: u_{g_{B}(\tau)} X\right]\right]+\frac{\partial}{\partial \tau} \phi\left[v_{g_{B}(\tau)} f: u_{g_{B}(\tau)} X\right]\right\} U_{B}^{\tau}(X)
$$

If the quantity (4.3) vanishes, the property (4.2) will be satisfied since it is obeyed at $\tau=0$. Making use of the group property $g_{B}(\tau+\delta \tau)=g_{B}(\delta \tau) g_{B}(\tau)$, we find that vanishing of expression (4.3) is equivalent to the property:

$$
\left.\frac{\partial}{\partial \tau}\right|_{\tau=0} \phi\left[v_{g_{B}(\tau)} f: u_{g_{B}(\tau)} X\right]-i[\phi[f: X] ; H(B: X)]=0 .
$$

We obtain the following lemma.

Lemma 4.1. Let the bilinear form (4.4) vanish on $\mathcal{D}$. Then the property (4.9) is satisfied on $\mathcal{D}$.

Proof. Consider the matrix element

$$
\chi^{\tau}=\left(U_{B}^{\tau}(X) \Psi_{1}, \phi\left[v_{g_{B}(\tau)} f: u_{g_{B}(\tau)} X\right] U_{B}^{\tau}(X) \Psi_{2}\right)-\left(\Psi_{1}, \hat{\phi}[f: X] \Psi_{2}\right),
$$

where $\Psi_{1}, \Psi_{2} \in \mathcal{D}$. Show it to be differentiable with respect to $\tau$. Let us check that for $\Psi \in \mathcal{D}$, the vector $\phi\left[v_{g_{B}(\tau)} f: u_{g_{B}(\tau)} X\right] \Psi$ is strongly continously differentiable with respect to $\tau$.

One has:

$$
\frac{\phi\left[v_{g_{B}(\tau+\delta \tau)} f: u_{g_{B}(\tau+\delta \tau)} X\right]-\phi\left[v_{g_{B}(\tau)} f: u_{g_{B}(\tau)} X\right]}{\delta \tau} \Psi=\phi\left[\frac{v_{g_{B}(\delta \tau)}-1}{\delta \tau} v_{g_{B}(\tau)} f: u_{g_{B}(\tau+\delta \tau)} X\right] \Psi+
$$

It follows from [48] that the first term tends to $\phi\left[\left.\frac{\partial}{\partial t}\right|_{t=0} v_{g_{B}(t)} v_{g_{B}(\tau)} f: u_{g_{B}(\tau)} X\right] \Psi$, while the second term tends to $\delta[B] \phi\left[v_{g_{B}(\tau)} f: u_{g_{B}(\tau)} X\right] \Psi$.

Notice that

$$
\begin{gathered}
\frac{\chi^{\tau+\delta \tau}-\chi^{\tau}}{\delta \tau}=\left(\phi\left[v_{g_{B}(\tau+\delta \tau)} f: u_{g_{B}(\tau+\delta \tau)} X\right] U_{B}^{\tau+\delta \tau}(X) \Psi_{1} ; \frac{U_{B}^{\tau+\delta \tau}(X)-U_{B}^{\tau}}{\delta \tau} \Psi_{2}\right) \\
+\left(U_{B}^{\tau+\delta \tau}(X) \Psi_{1} ;\left(\phi\left[v_{g_{B}(\tau+\delta \tau)} f: u_{g_{B}(\tau+\delta \tau)} X\right]-\phi\left[v_{g_{B}(\tau)} f: u_{g_{B}(\tau)} X\right]\right) U_{B}^{\tau}(X) \Psi_{2}\right) \\
+\left(\left(U_{B}^{\tau+\delta \tau}(X)-U_{B}^{\tau}(X)\right) \Psi_{1} ; \phi\left[v_{g_{B}(\tau)} f: u_{g_{B}(\tau)} X\right] U_{B}^{\tau}(X) \Psi_{2}\right) .
\end{gathered}
$$

This quantity tends as $\delta \tau \rightarrow 0$ to the matrix element of the bilinear form (4.3) and vanishes under condition (4.4). Lemma 4.1 is proved. 


\subsubsection{Check of invariance}

One should check property (4.2) for spatial translations and rotations, evolution and boost transformations.

For spatial translations and rotations, property (4.2) reads:

$$
\hat{\phi}(\mathbf{x}, t: X)=U_{0, \mathbf{a}, L}^{-1} \hat{\phi}\left(L \mathbf{x}+\mathbf{a}, t: u_{0, \mathbf{a}, L} X\right) U_{0, \mathbf{a}, L}
$$

It follows from commutativity of $U_{0, \mathbf{a}, L}$ and $U_{t}$ and table 3 that property (4.5) is satisfied.

For evolution operator, property (4.2) is rewritten as:

$$
\hat{\phi}(\mathbf{x}, t: X)=\left(U_{H}^{\tau}(X)\right)^{-1} \hat{\phi}\left(\mathbf{x}, t-\tau: u_{\tau} X\right) U_{H}^{\tau}(X)
$$

Relation (4.6) is a direct corollary of definition (4.1) and group property for evolution operators.

Consider now the $\mathbf{n}$-boost transformation. Check property (4.4). It can be presented as

$$
\left[\breve{B}^{k} ; \hat{\phi}(\mathbf{x}, t ; X)\right]=-i\left(x^{k} \frac{\partial}{\partial t}+t \frac{\partial}{\partial x^{k}}\right) \hat{\phi}(\mathbf{x}, t: X)
$$

or

$$
\begin{gathered}
{\left[B^{k}(X) ;\left(U_{H}^{t}(X)\right)^{-1} \phi\left(\mathbf{x}: u_{t} X\right) U_{H}^{t}(X)\right]+i \delta_{B}^{k}\left\{\left(U_{H}^{t}(X)\right)^{-1} \phi\left(\mathbf{x}: u_{t} X\right) U_{H}^{t}(X)\right\}=} \\
-i\left(x^{k} \frac{\partial}{\partial t}+t \frac{\partial}{\partial x^{k}}\right)\left(U_{H}^{t}(X)\right)^{-1} \phi\left(\mathbf{x}: u_{t} X\right) U_{H}^{t}(X)
\end{gathered}
$$

Let us make use of the property

$$
U_{H}^{t}(X) B^{k}(X)=i\left(\delta_{B}^{k} U_{H}^{t}\right)(X)+\left[B^{k}\left(u_{t} X\right)-t P^{k}\left(u_{t} X\right)\right] U_{H}^{t}(X) .
$$

which can be checked by multiplication by $\left(U_{H}^{t}(X)\right)^{-1}$ and differentiation with respect to $t$ in a weak sense (cf. 48). We take relation (4.7) to the form

$$
\left[\breve{B}^{k}(Y)-\breve{P}^{k}(Y) t ; \phi(\mathbf{x}: Y)\right]=x^{k}[\breve{H}(Y) ; \phi(\mathbf{x}: Y)]-i t \frac{\partial \phi(\mathbf{x}: Y)}{\partial x^{k}}
$$

where $Y=u_{t} X$. The property

$$
i \frac{\partial \phi(\mathbf{x}: Y)}{\partial x^{k}}=\left[\breve{P}^{k}(Y) ; \phi(\mathbf{x}: Y)\right]
$$

is a corollary of relation $(3.22)$. The relation

$$
\left[\breve{B}^{k}(Y)-x^{k} \breve{H}(Y) ; \phi(\mathbf{x}: Y)\right]=0
$$

is also checked by direct calculation.

Thus, we have obtained that the invariance property (2.5) is satisfied.

\section{$5 \quad$ Remarks on composed semiclassical states}

In the soliton quantization theory and in gauge field theories, the zero-mode problem arises [2]. To resolve it, one can consider the superposition of the "elementary" quantum field semiclassical states (1.3) of the form (cf. 36])

$$
\psi[\varphi(\cdot)]=\int \frac{d \alpha}{\lambda^{k / 4}} e^{\frac{i}{\lambda} S(\alpha)} e^{\frac{i}{\lambda} \Pi(\alpha ; \mathbf{x})[\varphi(\mathbf{x}) \sqrt{\lambda}-\Phi(\alpha, \mathbf{x})]} g\left(\alpha, \varphi(\cdot)-\frac{\Phi(\alpha, \cdot)}{\sqrt{\lambda}}\right)
$$

where $\alpha \in \mathbf{R}^{k}, S(\alpha), \Pi(\alpha ; \mathbf{x}), \Phi(\alpha ; \mathbf{x})$ are smooth functions. Calculate (formally) the functional integral for $(\psi, \psi)$ :

$$
\begin{gathered}
(\psi, \psi)=\int \frac{d \alpha d \gamma}{\lambda^{k / 2}} \int D \varphi e^{-\frac{i}{\lambda} S(\alpha)} e^{-\frac{i}{\lambda} \Pi(\alpha ; \mathbf{x})[\varphi(\mathbf{x}) \sqrt{\lambda}-\Phi(\alpha, \mathbf{x})]} g^{*}\left(\alpha, \varphi(\cdot)-\frac{\Phi(\alpha, \cdot)}{\sqrt{\lambda}}\right) \\
e^{\frac{i}{\lambda} S(\gamma)} e^{\frac{i}{\lambda} \Pi(\gamma ; \mathbf{x})[\varphi(\mathbf{x}) \sqrt{\lambda}-\Phi(\gamma, \mathbf{x})]} g\left(\gamma, \varphi(\cdot)-\frac{\Phi(\gamma, \cdot)}{\sqrt{\lambda}}\right)
\end{gathered}
$$


After substitution $\gamma=\alpha+\sqrt{\lambda} \beta, \varphi(\cdot)=\frac{\Phi(\alpha, \cdot)}{\sqrt{\lambda}}+\phi(\cdot)$ we obtain as $\lambda \rightarrow 0$ :

$$
\begin{gathered}
(\psi, \psi) \simeq \int d \alpha d \beta e^{\frac{i}{\sqrt{\lambda}} \beta_{s}\left(\frac{\partial S}{\partial \alpha_{s}}-\int d \mathbf{x} \Pi(\alpha, \mathbf{x}) \frac{\partial \Phi(\alpha, \mathbf{x})}{\partial \alpha_{s}}\right)} e^{\frac{i}{2} \beta_{s} \frac{\partial}{\partial \alpha_{s}}\left(\frac{\partial S}{\partial \alpha_{l}}-\int d \mathbf{x} \Pi(\alpha, \mathbf{x}) \frac{\partial \Phi(\alpha, \mathbf{x})}{\partial \alpha_{l}}\right) \beta_{l}} \\
\int D \phi g^{*}(\alpha, \phi(\cdot)) e^{i \beta_{l} \int d \mathbf{x}\left(\frac{\partial \Pi(\alpha, \mathbf{x})}{\partial \alpha_{l}} \phi(\mathbf{x})-\frac{\partial \Phi(\alpha, \mathbf{x})}{\partial \alpha_{l}} \frac{1}{i} \frac{\delta}{\delta \phi(\mathbf{x})}\right)} g(\alpha, \phi(\cdot))
\end{gathered}
$$

The condition

$$
\frac{\partial S}{\partial \alpha_{s}}=\int d \mathbf{x} \Pi(\alpha, \mathbf{x}) \frac{\partial \Phi(\alpha, \mathbf{x})}{\partial \alpha_{s}}
$$

should be satisfied. Otherwise, the integral (5.2) will be exponentially small as $\lambda \rightarrow 0$, so that state (5.1) will be trivial. Under condition (5.3), one has

$$
(\psi, \psi) \rightarrow_{\lambda \rightarrow 0} \int d \alpha d \beta \int D \phi g^{*}(\alpha, \phi(\cdot)) e^{i \beta_{l} \int d \mathbf{x}\left(\frac{\partial \Pi(\alpha, \mathbf{x})}{\partial \alpha_{l}} \phi(\mathbf{x})-\frac{\partial \Phi(\alpha, \mathbf{x})}{\partial \alpha_{l}} \frac{1}{i} \frac{\delta}{\delta \phi(\mathbf{x})}\right)} g(\alpha, \phi(\cdot))
$$

To specify the composed semiclassical state in the functional representation, one should:

(i) specify the smooth functions $(S(\alpha), \Pi(\alpha, \mathbf{x}), \Phi(\alpha, \mathbf{x})) \equiv X(\alpha)$ obeying eq.(5.3) (determine the $k$-dimensional isotropic manifold in the extended phase space $\mathcal{X})$;

(ii) specify the $\alpha$-dependent functional $g(\alpha, \phi(\cdot))$.

The inner product of composed semiclassical states is given by expression (5.4).

Since the inner product (5.4) may vanish for nonzero $g$, one should factorize the space of composed semiclassical states. Such functionals $g$ that obey the property

$$
\int d \alpha\left(g^{*}(\alpha, \cdot) \prod_{l} \delta\left[\int d \mathbf{x}\left(\frac{\partial \Pi(\alpha, \mathbf{x})}{\partial \alpha_{l}} \phi(\mathbf{x})-\frac{\partial \Phi(\alpha, \mathbf{x})}{\partial \alpha_{l}} \frac{1}{i} \frac{\delta}{\delta \phi(\mathbf{x})}\right)\right] g(\alpha, \cdot)=0\right.
$$

should be set to be equal to zero, $g \sim 0$.

One can define the Poincare tarnsformation of the composed semiclassical state as follows. The transformation of $(S(\alpha), \Pi(\alpha, \cdot), \Phi(\alpha, \cdot))$ is $u_{a, \Lambda}(S(\alpha), \Pi(\alpha, \cdot), \Phi(\alpha, \cdot))$. The transformation of $g(\alpha, \phi(\cdot))$ is

$$
\tilde{U}_{a, \Lambda}\left(u_{a, \Lambda}(S, \Pi, \Phi) \leftarrow(S, \Pi, \Phi)\right) g(\alpha, \phi(\cdot)) .
$$

One should check that the inner product entering to eq.(5.5) is invariant under Poincare transformations. This will also imply that equivalent states are taken to equivalent.

Since the functional Schrodinger representation is not well-defined, let us consider the Fock representation. One should then specify the $\alpha$-dependent Fock vector $Y(\alpha)=V^{-1} g(\alpha, \cdot)$ instead of the $\alpha$-dependent functional $g(\alpha, \phi(\cdot)$. Making use of formulas (2.1), we find that the inner product (5.4) takes the form

$$
\left(\left(\begin{array}{c}
\Lambda^{k} \\
Y(\cdot)
\end{array}\right),\left(\begin{array}{c}
\Lambda^{k} \\
Y(\cdot)
\end{array}\right)\right)=\int d \alpha d \beta\left(Y(\alpha), e^{\beta_{s} \int d \mathbf{x}\left(B_{s}(\alpha, \mathbf{x}) A^{+}(\mathbf{x})-B_{s}^{*}(\alpha, \mathbf{x}) A_{s}^{-}(\mathbf{x})\right)} Y(\alpha)\right)
$$

where

$$
B_{s}(\alpha, \cdot)=\hat{\Gamma}^{-1 / 2}\left(\hat{\mathcal{R}} \frac{\partial \Phi(\alpha, \cdot)}{\partial \alpha_{s}}-\frac{\partial \Pi(\alpha, \cdot)}{\partial \alpha_{s}}\right),
$$

$\hat{\Gamma}=\hat{\Gamma}(\Phi(\alpha, \cdot), \Pi(\alpha, \cdot)), \hat{\mathcal{R}}=\hat{\mathcal{R}}(\Phi(\alpha, \cdot), \Pi(\alpha, \cdot))$. If the isotropic manifold $(\Phi(\alpha, \cdot), \Pi(\alpha, \cdot))$ is nondegenerate, the functions $B_{s}(\alpha, \mathbf{x})$ are linearly independent.

The Poincare transformation of the composed semiclassical state

$$
\left(\begin{array}{c}
\{X(\alpha)\} \\
Y(\alpha)
\end{array}\right)
$$

is

$$
\left(\begin{array}{c}
\left\{u_{a, \Lambda} X(\alpha)\right\} \\
U_{a, \Lambda}\left(u_{a, \Lambda} X(\alpha) \leftarrow X(\alpha)\right) Y(\alpha)
\end{array}\right)
$$


Consider the quantity

$$
\begin{gathered}
\left.\left(B_{s}, B_{l}\right)-\left(B_{l}, B_{s}\right)=\left(\frac{\partial \Phi}{\partial \alpha_{s}}\right),\left(\hat{\mathcal{R}}^{*}-\hat{\mathcal{R}}\right) \hat{\Gamma}^{-1} \frac{\partial \Pi}{\partial \alpha_{l}}\right)-\left(\frac{\partial \Phi}{\partial \alpha^{\prime}},\left(\hat{\mathcal{R}}^{*}-\hat{\mathcal{R}}\right) \hat{\Gamma}^{-1} \frac{\partial \Pi}{\partial \alpha_{s}}\right)= \\
i \int d \mathbf{x}\left(\frac{\partial \Phi(\alpha, \mathbf{x})}{\partial \alpha_{l}} \frac{\partial \Pi(\alpha, \mathbf{x})}{\partial \alpha_{s}}-\frac{\partial \Phi(\alpha, \mathbf{x})}{\partial \alpha_{s}} \frac{\partial \Pi(\alpha, \mathbf{x})}{\partial \alpha_{l}}\right) .
\end{gathered}
$$

Differentiating (5.3) with respect to $\alpha_{l}$, we obtain that quantity (5.8) vanishes. Thus, operators $\beta_{s} \int d \mathbf{x}\left(B_{s}(\alpha, \mathbf{x}) A^{+}(\mathbf{x})-B_{s}^{*}(\alpha, \mathbf{x}) A_{s}^{-}(\mathbf{x})\right)$ commute each other.

It follows from the resulats of 48 that the inner product (5.7) is correctly defined, while Poincare transformations of composed semcialssical states satisfy the group property and conserve the inner product (5.7).

\section{Conclusions}

In this paper a notion of a semiclassical state is introduced. "Elementary" semiclassical state are specified by a set $(X, \Psi)$ of classical field configuration $X$ (point on the infinite-dimensional manifold $\mathcal{X}$, see section 2 and subsection 3.2) and element $\Psi$ of the space $\mathcal{F}$. Set of all "elementary" semiclassical states may be viewed as a semiclassical bundle.

The physical meaning of classical field $X$ is evident. Discuss the role of $\Psi$. In the soliton quantization language [1, 2] $\Psi$ specifies whether the quantum soliton is in the ground or excited state. For the Gaussian approach [14, 15, 16, 17], $\Psi$ specifies the form of the Gaussian functional, while for QFT in the strong external classical field [6, 7] $\Psi$ is a state of a quantum field in the classical background.

The "composed" semiclassical states have been also introduced (section 5). They can be viewed as superpositions of "elementary" semiclassical states and are specified by the functions $(X(\tau), \Psi(\tau))$ defined on some domain of $\mathbf{R}^{k}$ with values on the semiclassical bundle.

Not arbitrary superposition of elementary semiclassical states is nontrivial. The isotropic condition (5.3) should be satisfied. Moreover, the inner product of the "composed" semiclassical states (eq.(5.6) is degenerate, so that there is a "gauge freedom" (5.5) in specifying composed semiclassical states.

The composed semiclassical states are used [36] in soliton quantization, since there are translation zero modes and solitons can be shifted. They are useful if there are conserved integrals of motion like charges. The correspondence between composed and elementary semiclassical states in QFT resembles the relationship between WKB and wave packet approximations in quantum mechanics.

An important feature of QFT is the property of Poincare invariance. In this paper an explicit check of this property is presented for semiclassical QFT. The Poincare transformations of elementary and composed semiclassical states have been constructed as follows. First, the simplest Poincare transformations like spatial translations and rotations, evolution and boost are considered. The infinitesimal transformations are investigated, the Lie algebraic commutation relations have been checked and the group properties have been justified.

For the "composed" states, conservation of the degenerate inner product and isotropic condition under Poincare transformation have been checked.

An important feature of QFT is a notion of field. In this paper this notion is introduced for semiclassical QFT. The property of Poincare invariance of semiclassical field is checked.

This work was supported by the Russian Foundation for Basic Research, projects 99-01-01198 and 01-01-06251.

\section{A Weyl calculus}

The purpose of this appendix is to investigate some properties of Weyl symbols of operators which are useful in justification of properties P1-P6. 


\section{A.1 Definition of Weyl symbol}

Firs of all, remind the definition of Weyl symbol of operator (see, for example, [34, 51). Let $A(x, k), x, k \in \mathbf{R}^{d}$ be a classical observable depending on coordinates $x=\left(x_{1}, \ldots, x_{d}\right)$ and momenta $k=\left(k_{1}, \ldots, k_{d}\right)$. To specify the corresponding quantum observable $\hat{A}$ (to "quantize" the observable $A$ ), one should present it as a superposition of exponents,

$$
A(x, k)=\int d \alpha d \beta \tilde{A}(\alpha, \beta) e^{i \alpha k+i \beta x}
$$

and set

$$
\hat{A}=\int d \alpha d \beta \tilde{A}(\alpha, \beta) e^{i \alpha \hat{k}+i \beta \hat{x}}
$$

Applying the formula for inverse Fourier transformation, we find

$$
(\hat{A} f)(x)=\int \frac{d \alpha d p}{(2 \pi)^{d}} A\left(x+\frac{\alpha}{2} ; p\right) e^{-i \alpha p} f(x+\alpha) .
$$

We denote the operator $\hat{A}$ of the form (A.1) as $\hat{A}=\mathcal{W}(A)$. We will also write $A=\overline{\mathcal{W}}(\hat{A})$ if $\hat{A}=\mathcal{W}(A)$.

Definition A.1. The operator $\mathcal{W}(A)$ is called a Weyl quantization of the function $A$. The function $\overline{\mathcal{W}}(\hat{A})$ is called as a Weyl symbol of the operator $\hat{A}$.

\section{A.2 Some calsses of Weyl symbols}

\section{A.2.1 Classes $\mathcal{A}_{N}$ and $\mathcal{B}_{N}$}

For investigations of QFT ultraviolet divergences, we are interested in behavior of Weyl symbols of operators at large values of momenta. Let us introduce some important spaces. Let $\omega_{k}=\sqrt{k^{2}+m^{2}}$ for some $m$.

Definition A.2. 1. We say that a smooth function $A(x, k)$ is of the class $\mathcal{B}_{N}$ if and only if the functions

$$
\omega_{k}^{N+s} \frac{\partial^{s} A}{\partial k^{i_{1}} \ldots \partial k^{i_{s}}}
$$

are bounded for all $s, i_{1}, \ldots, i_{s}$.

2. Let $A_{n} \in \mathcal{B}_{N}, n=\overline{1, \infty}, A \in \mathcal{B}_{N}$. We say that $\mathcal{B}_{N}-\lim _{n \rightarrow \infty} A_{n}=A$ if and only if

$$
\lim _{n \rightarrow \infty} \max _{k, x} \omega_{k}^{N+s} \frac{\partial^{s}\left(A_{n}-A\right)}{\partial k^{i_{1}} \ldots \partial k^{i_{s}}}=0
$$

for all $s, i_{1}, \ldots, i_{s}$.

3. We say that a function $A \in \mathcal{B}_{N}$ is of the class $\mathcal{A}_{N}$ if and only if

$$
x_{j_{1} \ldots x_{j_{R}}} \frac{\partial}{\partial x_{s_{1}}} \ldots \frac{\partial}{\partial x_{s_{P}}} A \in \mathcal{B}_{N}
$$

for all $R, P, j_{1}, \ldots, j_{R}, s_{1}, \ldots, s_{P}$.

4. Let $A_{n} \in \mathcal{A}_{N}, A \in \mathcal{A}_{N}$. We say that $\mathcal{A}_{N}-\lim _{n \rightarrow \infty} A_{n}=A$ if and only if

$$
\mathcal{B}_{N}-\lim _{n \rightarrow \infty} x_{j_{1}} \ldots x_{j_{R}} \frac{\partial}{\partial x_{s_{1}}} \ldots \frac{\partial}{\partial x_{s_{P}}}\left(A_{n}-A\right)=0
$$

for all $R, P, j_{1}, \ldots, j_{R}, s_{1}, \ldots, s_{P}$.

Let us investigate some properties of introduced classes $\mathcal{A}_{N}$ and $\mathcal{B}_{N}$.

Lemma A.1. 1. $\mathcal{A}_{N+R} \subseteq \mathcal{A}, \mathcal{B}_{N+R} \subseteq \mathcal{B}$ for $R \geq 0$.

2. Let $\mathcal{A}_{N+R}-\lim _{n \rightarrow \infty} A_{n}=A$ and $R \geq 0$. Then $\mathcal{A}_{N}-\lim _{n \rightarrow \infty} A_{n}=A$.

3. Let $\mathcal{B}_{N+R}-\lim _{n \rightarrow \infty} A_{n}=A$ and $R \geq 0$. Then $\mathcal{B}_{N}-\lim _{n \rightarrow \infty} A_{n}=A$. 
The proof is obvious: it is sufficient to notice that $\omega_{k}^{-R}$ is a bounded function.

Lemma A.2. 1. Let $A \in \mathcal{B}_{N}$. Then $\frac{\partial}{\partial k_{i}} A \in \mathcal{B}_{N+1}$.

2. Let $A \in \mathcal{A}_{N}$. Then $x_{i} A \in \mathcal{A}_{N}, \frac{\partial A}{\partial x_{i}} \in \mathcal{A}_{N}, \frac{\partial}{\partial k_{i}} A \in \mathcal{A}_{N+1}, f(x) A \in \mathcal{A}_{N}$ for smooth bounded function $f(x)$.

3. Let $\mathcal{B}_{N}-\lim _{n \rightarrow \infty} A_{n}=A$. Then $\mathcal{B}_{N+1}-\lim _{n \rightarrow \infty} \frac{\partial}{\partial k_{i}} A_{n}=\frac{\partial}{\partial k_{i}} A$.

4. Let $\mathcal{A}_{N}-\lim _{n \rightarrow \infty} A_{n}=A$. Then $\mathcal{A}_{N}-\lim _{n \rightarrow \infty} x_{i} A_{n}=x_{i} A, \mathcal{A}_{N}-\lim _{n \rightarrow \infty} \frac{\partial A_{n}}{\partial x_{i}}=\frac{\partial A}{\partial x_{i}}$, $\mathcal{A}_{N+1}-\lim _{n \rightarrow \infty} \frac{\partial}{\partial k_{i}} A_{n}=\frac{\partial}{\partial k_{i}} A, \mathcal{A}_{N}-\lim _{n \rightarrow \infty} f(x) A_{n}=f(x) A$ for smooth bounded function $f(x)$.

The proof is also obvious.

Lemma A.3. Let $A_{1} \in \mathcal{B}_{N_{1}}, A_{2} \in \mathcal{B}_{N_{2}}$. Then $A_{1} A_{2} \in \mathcal{B}_{N_{1}+N_{2}}$.

Proof. It is sufficient to check that the expression

$$
\omega_{k}^{N_{1}} \omega_{k}^{N_{2}} \omega_{k}^{s} \frac{\partial^{s}}{\partial k_{i_{1}} \ldots \partial k_{i_{s}}}\left(A_{1} A_{2}\right)
$$

is bounded. This statement is a corollary of properties $A_{1} \in \mathcal{B}_{N_{1}}, A_{2} \in \mathcal{B}_{N_{2}}$ and formula

$$
\frac{\partial}{\partial k_{i}}(f g)=\frac{\partial}{\partial k_{i}} f \cdot g+f \cdot \frac{\partial}{\partial k_{i}} g \text {. }
$$

Lemma A.3 is proved.

Lemma A.4. The following properties are satisfied: $k_{i} \in \mathcal{B}_{-1}, \omega_{k}^{\alpha} \in \mathcal{B}_{-\alpha}$.

Proof. Since $\left|k_{i} / \omega_{k}\right|<1$, we obtain the property $k_{i} \in \mathcal{B}_{-1}$. For the function $\omega_{k}^{\alpha}$, one has

$$
\frac{\partial}{\partial k_{i_{1}}} \ldots \frac{\partial}{\partial k_{i_{s}}} \omega_{k}^{\alpha}=\omega_{k}^{\alpha} \mathcal{P}\left(k_{i} / \omega_{k}\right)
$$

where $\mathcal{P}$ is a polynomial in $k_{i} / \omega_{k}$. Property (A.3) is checked by induction. Therefore, functions (A.2) are bounded for $N=1$. Lemma A.4 is proved.

Lemma A.5. 1. Let $A \in \mathcal{B}_{N}$. Then

$$
k_{i} A \in \mathcal{B}_{N-1}, \quad \omega_{k}^{-\alpha} A \in \mathcal{B}_{N+\alpha}, \quad \frac{\partial A}{\partial k_{i}} \in \mathcal{B}_{N+1} .
$$

2. Let $A \in \mathcal{A}_{N}$. Then

$$
k_{i} A \in \mathcal{A}_{N-1}, \quad \omega_{k}^{-\alpha} A \in \mathcal{A}_{N+\alpha}, \quad \frac{\partial A}{\partial k_{i}} \in \mathcal{A}_{N+1} .
$$

Proof. Property 1 is a corollary of lemmas A.2 and A.4. Property 1 implies property 2. Lemma is proved.

Lemma A.6. 1. Let $\mathcal{B}_{N}-\lim _{n \rightarrow \infty} A_{n}=A$. Then

$$
\mathcal{B}_{N-1}-\lim _{n \rightarrow \infty} k_{i} A_{n}=k_{i} A, \quad \mathcal{B}_{N+\alpha}-\lim _{n \rightarrow \infty} \omega_{k}^{-\alpha} A_{n}=\omega_{k}^{-\alpha} A, \quad \mathcal{B}_{N+1}-\lim _{n \rightarrow \infty} \frac{\partial A_{n}}{\partial k_{i}}=\frac{\partial A}{\partial k_{i}}
$$

2. Let $\mathcal{A}_{N}-\lim _{n \rightarrow \infty} A_{n}=$ A. Then

$$
\mathcal{A}_{N-1}-\lim _{n \rightarrow \infty} k_{i} A_{n}=k_{i} A, \quad \mathcal{A}_{N+\alpha}-\lim _{n \rightarrow \infty} \omega_{k}^{-\alpha} A_{n}=\omega_{k}^{-\alpha} A, \quad \mathcal{A}_{N+1}-\lim _{n \rightarrow \infty} \frac{\partial A_{n}}{\partial k_{i}}=\frac{\partial A}{\partial k_{i}} .
$$

The proof is analogous to the proof of lemma A.3.

Lemma A.7. 1. Let $A_{1} \in \mathcal{A}_{N_{1}}, A_{2} \in \mathcal{A}_{N_{2}}$. Then $A_{1} A_{2} \in \mathcal{A}_{N_{1}+N_{2}}$.

2. Let $\mathcal{A}_{N_{1}}-\lim _{n \rightarrow \infty} A_{1, n}=A_{1}, \mathcal{A}_{N_{2}}-\lim _{n \rightarrow \infty} A_{2, n}=A_{2}$. Then $\mathcal{A}_{N_{1}+N_{2}}-\lim _{n \rightarrow \infty} A_{1, n} A_{2, n}=A_{1} A_{2}$.

The proof is analogous to lemma A.3. 


\section{A.2.2 Properties of operators and symbols}

Lemma A.8. 1. Let $A \in \mathcal{A}_{0}$. Then the operator $\mathcal{W}(A)$ A.1 is bounded.

2. Let $\mathcal{A}_{0}-\lim _{n \rightarrow \infty} A_{n}=0$. Then $\lim _{n \rightarrow \infty}\left\|\mathcal{W}\left(A_{n}\right)\right\|=0$.

Proof (cf. [36]). Let us obtain an estimation for the norm $\|\hat{A}\|$. One has

$$
\hat{A}=\int d \beta e^{i \beta \hat{x}} \int d \alpha e^{i \alpha(\hat{k}+\beta / 2)} \tilde{A}(\alpha, \beta) .
$$

The estimation $\left\|\int d \beta \hat{F}(\beta)\right\| \leq \int d \beta\|\hat{F}(\beta)\|$ implies

$$
\|\hat{A}\| \leq \int d \beta \| \int d \alpha e^{i \alpha(\hat{k}+\beta / 2)} \tilde{A}(\alpha, \beta) .
$$

However, for operator $F(\hat{k})$ one has $\|F(\hat{k})\|=\sup _{k}\|F(k)\|$, since in the momentum representation $F(\hat{k})$ is the operator of multiplication be $F(k)$. Therefore,

$$
\begin{gathered}
|| \int d \alpha e^{i \alpha(\hat{k}+\beta / 2)} \tilde{A}(\alpha, \beta) \|=\max _{k}\left|\int d \alpha e^{i \alpha(k+\beta / 2)} \tilde{A}(\alpha, \beta)\right|=\max _{k}\left|\int d \alpha e^{i \alpha k} \tilde{A}(\alpha, \beta)\right|= \\
\max _{k}\left|\int \frac{d x}{(2 \pi)^{d}} A(x, k) e^{-i \beta x}\right|=\frac{1}{\left(\beta^{2}+1\right)^{N}} \max _{k}\left|\int \frac{d x}{(2 \pi)^{d}\left(x^{2}+1\right)^{N}} e^{-i \beta x}\left(x^{2}+1\right)^{N}\left(-\Delta_{x}+1\right)^{N} A(x, k)\right| .
\end{gathered}
$$

Here $N$ is an arbitrary number such that $N>d / 2$. Thus,

$$
\|\hat{A}\| \leq \frac{1}{(2 \pi)^{d}} \int \frac{d \beta d x}{\left(\beta^{2}+1\right)^{N}\left(x^{2}+1\right)^{N}} \max _{k x}\left|\left(x^{2}+1\right)^{N}\left(-\Delta_{x}+1\right)^{N} A(x, k)\right| .
$$

The first statement is judtified. Proof of the second statement is analogous. Lemma A.8 is proved.

Lemma A.9.1. Let $A \in \mathcal{A}_{N}, N>d / 2$. Then $\mathcal{W}(A)$ is a Hilbert-Schmidt operator.

2. Let $\mathcal{A}_{N}-\lim _{n \rightarrow \infty} A_{n}=0, N>d / 2$. Then $\lim _{n \rightarrow \infty}\left\|\mathcal{W}\left(A_{n}\right)\right\|_{2}=0$.

Proof. Let us use the property [34, 51]

$$
\|\hat{A}\|_{2}^{2}=\int \frac{d x d k}{(2 \pi)^{d}}|A(x, k)|^{2}
$$

whcih can be obtained from definition (A.1). One has

$$
\|\hat{A}\|_{2}^{2} \leq \int \frac{d x}{(2 \pi)^{d}\left(x^{2}+1\right)^{N}} \frac{d k}{\omega_{k}^{2 N}} \max _{x k}\left|\left(x^{2}+1\right)^{N / 2} \omega_{k}^{N} A(x, k)\right|^{2} .
$$

The first statement is justified. Proof of the second statement is analogous. Lemma A.9 is proved.

\section{A.3 Properties of $*$-product}

Remind that the Weyl sumbol of the product of operators

$$
A * B=\overline{\mathcal{W}}(\mathcal{W}(A) \mathcal{W}(B))
$$

can be presented as [34, 51]

$$
(A * B)(x, k)=\int \frac{d \beta_{1} d \beta_{2} d \xi_{1} d \xi_{2}}{(2 \pi)^{2 d}} A\left(x+\xi_{1}, k+\frac{\beta_{2}}{2}\right) B\left(x+\xi_{2}, k-\frac{\beta_{1}}{2}\right) e^{-i \beta_{1} \xi_{1}-i \beta_{2} \xi_{2}}
$$

Formula (A.4) can be obtained from definition (A.1).

Let us investigate some properties of formula (A.4). Let us find an expansion of formula (A.4) as $|k| \rightarrow \infty$. Formally, one has

$$
\begin{aligned}
& A\left(x+\xi_{1}, k+\frac{\beta_{2}}{2}\right)=\sum_{n_{2}=0}^{\infty} \frac{1}{2^{n_{2} n_{2} !} !} \frac{\partial^{n_{2}} A\left(x+\xi_{1}, k\right)}{\partial k^{i_{1}} \ldots \partial k^{i} n_{2}} \beta_{2}^{i_{1}} \ldots \beta_{2}^{i_{n_{2}}} \\
& B\left(x+\xi_{2}, k-\frac{\beta_{1}}{2}\right)=\sum_{n_{1}=0}^{\infty} \frac{(-1)^{n_{1}}}{2^{n_{1} n_{1} !}} \frac{\partial^{n_{1}} B\left(x+\xi_{2}, k\right)}{\partial k^{j_{1} \ldots \partial k^{j} n_{1}}} \beta_{1}^{j_{1}} \ldots \beta_{1}^{j_{n_{1}}} .
\end{aligned}
$$


Therefore,

$$
\begin{gathered}
(A * B)(x, k)=\sum_{n_{1} n_{2}=0}^{\infty} \frac{(-1)^{n_{1}}}{2^{n_{1}+n_{2} n_{1} ! n_{2} !}} \int \frac{d \beta_{1} d \beta_{2} d \xi_{1} d \xi_{2}}{(2 \pi)^{2 d}} e^{-i \beta_{1} \xi_{1}-i \beta_{2} \xi_{2}} \frac{\partial^{n_{2}} A\left(x+\xi_{1}, k\right)}{\partial k^{i_{1}} \ldots \partial k^{i} n_{2}} \beta_{2}^{i_{1}} \ldots \beta_{2}^{i_{n_{2}}} \\
\times \frac{\partial^{n_{1}} B\left(x+\xi_{2}, k\right)}{\partial k^{j_{1}} \ldots \partial k^{j n_{1}}} \beta_{1}^{j_{1}} \ldots \beta_{1}^{j_{n_{1}}}=\sum_{n_{1} n_{2}=0}^{\infty} \frac{{ }^{n_{1}} n_{2}}{2^{n_{1}+n_{2} n_{1} ! n_{2} !}} \frac{\partial^{n_{1}+n_{2}} A(x, k)}{\partial k^{i_{1}} \ldots \partial k^{i_{2}} \partial x^{j_{1}} \ldots \partial x^{j n_{1}}} \frac{\partial^{n_{1}+n_{2}} B(x, k)}{\partial x^{i_{1}} \ldots \partial x^{i_{2}} \partial k^{j_{1}} \ldots \partial k^{j n_{1}}}
\end{gathered}
$$

Denote

$$
\left(A *{ }^{K} B\right)(x, k)=\sum_{n_{1} n_{2} \geq 0, n_{1}+n_{2} \leq K} \frac{i^{n_{1}-n_{2}}}{2^{n_{1}+n_{2}} n_{1} ! n_{2} !} \frac{\partial^{n_{1}+n_{2}} A(x, k)}{\partial k^{i_{1}} \ldots \partial k^{i_{2}} \partial x^{j_{1}} \ldots \partial x^{j_{n_{1}}}} \frac{\partial^{n_{1}+n_{2}} B(x, k)}{\partial x^{i_{1}} \ldots \partial x^{i_{2}} \partial k^{j_{1}} \ldots \partial k^{j_{n_{1}}}}
$$

This is an asymptotic expansion in $1 /|k|$ as $|k| \rightarrow \infty$. Let us estimate an accuracy of the asymptotic series.

Making use of the relation

$$
A\left(x+\xi_{1}, k+\frac{\beta_{2}}{2}\right)-A\left(x+\xi_{1}, k\right)=\int_{0}^{1} d\left(\alpha_{2}-1\right) \frac{\partial}{\partial \alpha_{2}} A\left(x+\xi_{1}, k+\alpha_{2} \frac{\beta_{2}}{2}\right)
$$

and integrating by parts $N_{2}$ times, we find

$$
\begin{gathered}
A\left(x+\xi_{1}, k+\frac{\beta_{2}}{2}\right)=\sum_{n_{2}=0}^{N_{2}} \frac{1}{2^{n_{2} n_{2} !}} \frac{\partial^{n_{2}} A\left(x+\xi_{1}, k\right)}{\partial k^{i_{1}} \ldots \partial k^{i_{n}}} \beta_{2}^{i_{1}} \ldots \beta_{2}^{i_{n_{2}}} \\
\quad+\int_{0}^{1} d \alpha_{2} \frac{\left(1-\alpha_{2}\right)^{N_{2}}}{2^{N_{2}+1} N_{2} !} \frac{\partial^{N_{2}+1} A\left(x+\xi_{1}, k+\alpha_{2} \frac{\beta_{2}}{2}\right)}{\partial k^{i_{1}} \ldots \partial k^{i_{N}+1}} \beta_{2}^{i_{1}} \ldots \beta_{2}^{i_{N_{2}+1}} .
\end{gathered}
$$

Analogously,

$$
\begin{aligned}
& B\left(x+\xi_{2}, k-\frac{\beta_{1}}{2}\right)=\sum_{n_{1}=0}^{N_{1}} \frac{(-1)^{n_{1}}}{2^{n_{1}} n_{1} !} \frac{\partial^{n_{1}} A\left(x+\xi_{2}, k\right)}{\partial k^{i_{1} \ldots \partial \partial k^{i} n_{1}}} \beta_{1}^{i_{1}} \ldots \beta_{1}^{i_{n_{2}}} \\
& +\int_{0}^{1} d \alpha_{1} \frac{\left(1-\alpha_{1}\right)^{N_{1}}(-1)^{N_{1}}}{2^{N_{1}+1} N_{1} !} \frac{\partial^{N_{1}+1} B\left(x+\xi_{2}, k-\alpha_{1} \frac{\beta_{1}}{2}\right)}{\partial k^{i_{1}} \ldots \partial k^{i_{1}+1}} \beta_{1}^{i_{1}} \ldots \beta_{1}^{i_{N_{1}+1}} .
\end{aligned}
$$

Therefore,

$$
\begin{gathered}
(A * B)(x, k)=\sum_{n_{1}=0}^{N_{1}} \sum_{n_{2}=0}^{N_{2}} \frac{i^{n_{1}-n_{2}}}{2^{n_{1}+n_{2} n_{1} ! n_{2} !}} \frac{\partial^{n_{1}+n_{2}} A(x, k)}{\partial k^{i_{1}} \ldots \partial k^{i_{2}}{ }^{j_{2}} \partial x^{j_{1} \ldots} \ldots x^{j n_{1}}} \frac{\partial^{n_{1}+n_{2}} B(x, k)}{\partial x^{i_{1}} \ldots \partial x^{i_{2}} \partial k^{j_{1}} \ldots \partial k^{j n_{1}}} \\
+\sum_{n_{1}=0}^{N_{1}} r_{n_{1} N_{2}}^{(1)}+\sum_{n_{2}=0}^{\infty} r_{N_{1} n_{2}}^{(2)}+R_{N_{1} N_{2}}
\end{gathered}
$$

with the following remaining terms,

$$
\begin{aligned}
& r_{n_{1} N_{2}}^{(1)}=\int \frac{d \beta_{1} d \beta_{2} d \xi_{1} d \xi_{2}}{(2 \pi)^{2 d}} e^{-i \beta_{1} \xi_{1}-i \beta_{2} \xi_{2}} \int_{0}^{1} d \alpha_{2} \frac{\left(1-\alpha_{2}\right)^{N_{2}}}{2^{N_{2}+1} N_{2} !} \frac{\partial^{N_{2}+1} A\left(x+\xi_{1}, k+\alpha_{2} \frac{\beta_{2}}{2}\right)}{\partial k^{i_{1}} \ldots \partial k^{i N_{2}+1}} \beta_{2}^{i_{1}} \ldots \beta_{2}^{i_{N_{2}+1}} \\
& \times \frac{(-1)^{n_{1}}}{2^{n_{1}} n_{1} !} \frac{\partial^{n_{1}} B\left(x+\xi_{1}, k\right)}{\partial k^{j_{1}} \ldots \partial k^{j_{1}}} \beta_{1}^{j_{1}} \ldots \beta_{1}^{j_{n_{1}}} \\
& r_{N_{1} n_{2}}^{(2)}=\int \frac{d \beta_{1} d \beta_{2} d \xi_{1} d \xi_{2}}{(2 \pi)^{2 d}} e^{-i \beta_{1} \xi_{1}-i \beta_{2} \xi_{2}} \int_{0}^{1} d \alpha_{1} \frac{\left(1-\alpha_{1}\right)^{N_{1}}(-1)^{N_{1}+1}}{2^{N_{1}+1} N_{1} !} \frac{\partial^{N_{1}+1} B\left(x+\xi_{2}, k-\alpha_{1} \frac{\beta_{1}}{2}\right)}{\partial k^{j_{1}} \ldots \partial k^{j_{1}+1}} \beta_{1}^{j_{1}} \ldots \beta_{1}^{j_{N_{1}+1}} \\
& \times \frac{1}{2^{n_{2} n_{2} !}} \frac{\partial^{n_{2} B\left(x+\xi_{2}, k\right)}}{\partial k^{i_{1}} \ldots \partial k^{i_{2}}{ }_{2}} \beta_{2}^{i_{1}} \ldots \beta_{2}^{i_{n_{2}}} \text {; } \\
& R_{N_{1} N_{2}}=\int \frac{d \beta_{1} d \beta_{2} d \xi_{1} d \xi_{2}}{(2 \pi)^{2 d}} e^{-i \beta_{1} \xi_{1}-i \beta_{2} \xi_{2}} \int_{0}^{1} d \alpha_{2} \frac{\left(1-\alpha_{2}\right)^{N_{2}}}{2^{N_{2}+1} N_{2} !} \frac{\partial^{N_{2}+1} A\left(x+\xi_{1}, k+\alpha_{2} \frac{\beta_{2}}{2}\right)}{\partial k^{i_{1}} \ldots \partial k^{i_{N}+1}} \beta_{2}^{i_{1}} \ldots \beta_{2}^{i_{N_{2}+1}} \\
& \times \int_{0}^{1} d \alpha_{1} \frac{\left(1-\alpha_{2}\right)^{N_{2}}(-1)^{N_{1}+1}}{2^{N_{1}+1} N_{1} !} \frac{\partial^{N_{1}+1} B\left(x+\xi_{2}, k-\alpha_{1} \frac{\beta_{1}}{2}\right)}{\partial k^{j_{1}} \ldots \partial k^{j_{N_{1}+1}}} \beta_{1}^{j_{1}} \ldots \beta_{1}^{j_{N_{1}+1}} .
\end{aligned}
$$

Let us investigate the remaining terms.

\section{A.3.1 The $k$-independent case}

Definition A.3. We say that the function $f(x), x \in \mathbf{R}^{d}$ is of the class $\mathcal{C}$ if $f$ is a smooth function such that for each set $\left(i_{1}, \ldots, i_{l}\right)$ there exists $m>0$ such that the function

$$
\left(x^{2}+1\right)^{-m} \frac{\partial^{l}}{\partial x^{i_{1}} \ldots \partial x^{i_{l}}} f
$$

is bounded. 
Let $A=f(x), f \in \mathcal{C}$. Then the only nontrivial term is $r_{N_{1} 0}^{(2)}$ which is taken by integrating by parts to the form

$$
r_{N_{1} 0}^{(2)}=\int \frac{d \beta_{1} d \xi_{1}}{(2 \pi)^{d}} e^{-i \beta_{1} \xi_{1}} \frac{\partial^{N_{1}+1}}{\partial x^{j_{1}} \ldots \partial x^{j_{N_{1}+1}}} f\left(x+\xi_{1}\right) \int_{0}^{1} d \alpha_{1}\left(\frac{i}{2}\right)^{N_{1}+1} \frac{\left(1-\alpha_{1}\right)^{N_{1}}}{N_{1} !} \frac{\partial^{N_{1}+1} B\left(x, k-\alpha_{1} \frac{\beta_{1}}{2}\right)}{\partial k^{j_{1}} \ldots \partial k^{j_{N_{1}+1}}} .
$$

Let us prove some auxiliary statements.

Lemma A.10. For some constent $A_{1}$ the estimation

$$
\omega_{k} \leq A_{1} \omega_{p} \omega_{k-p}
$$

is satisfied.

Proof. Let $p=\left(\frac{1}{2}+\alpha\right) k+p_{\perp}, \alpha \in \mathbf{R}, p_{\perp} \perp k$. Then

$$
\frac{\omega_{k}}{\omega_{p} \omega_{k-p}} \leq \frac{\omega_{k}}{\omega_{(1 / 2+\alpha) k} \omega_{(1 / 2-\alpha) k}} \equiv f(\alpha, k)
$$

so that it is sufficient to check estimation (A.7) for $p=\alpha k$ only. For the function $1 / f^{2}$, one has

$$
\frac{1}{f^{2}(\alpha, k)}=\frac{1}{k^{2}+m^{2}}\left[\left(\frac{1}{2}+\alpha\right)^{2} k^{2}+m^{2}\right]\left[\left(\frac{1}{2}-\alpha\right)^{2} k^{2}+m^{2}\right] .
$$

It has the following minimal value

$$
\min _{\alpha} \frac{1}{f^{2}(\alpha, k)}=\left\{\begin{array}{c}
\frac{\left(k^{2} / 4+m^{2}\right)^{2}}{k^{2}+m^{2}}, \quad k^{2}<4 m^{2}, \quad \alpha=0 . \\
\frac{k^{2} m^{2}}{k^{2}+m^{2}}, \quad k^{2}>4 m^{2}, \quad \alpha=\sqrt{\frac{1}{4}-\frac{m^{2}}{k^{2}}}
\end{array}\right.
$$

The quantity (A.8) is bounded below. Thus, lemma is proved.

Corollary. For $0<\gamma<1$,

$$
\frac{\omega_{k}}{\omega_{p} \omega_{k-\gamma p}} \leq A_{1}
$$

Lemma A.11. Let $C \in \mathcal{A}_{N}, \chi \in \mathcal{C}, \varphi \in C[0,1]$. Then for

$$
F(x, k)=\int_{0}^{1} d \alpha \varphi(\alpha) \int \frac{d \beta d \xi}{(2 \pi)^{d}} e^{-i \beta \xi} \chi(x+\xi) C\left(x, k-\frac{\alpha \beta}{2}\right)
$$

the function $\omega_{k}^{N} F$ is bounded.

Proof. Inserting the identity

$$
e^{-i \beta \xi}=\left(\xi^{2}+1\right)^{-L_{1}}\left(-\frac{\partial^{2}}{\partial \beta^{2}}+1\right)^{L_{1}} e^{-i \beta \xi}
$$

and integrating by parts, we obtain that

$$
F(x, k)=\int_{0}^{1} d \alpha \varphi(\alpha) \int \frac{d \beta d \xi}{(2 \pi)^{d}} \frac{1}{\left(\xi^{2}+1\right)^{L_{1}}} e^{-i \beta \xi} \chi(x+\xi)\left(1-\frac{\alpha^{2}}{4} \frac{\partial^{2}}{\partial k^{2}}\right)^{L_{1}} C\left(x ; k-\frac{\alpha \beta}{2}\right) .
$$

For the function $\omega_{k}^{N} F$, one has

$$
\begin{aligned}
\omega_{k}^{N} F=\int_{0}^{1} d \alpha \varphi(\alpha) \int \frac{d \beta d \xi}{(2 \pi)^{d}} \frac{1}{\left(\xi^{2}+1\right)^{L_{1} \omega_{\text {beta/2 }}^{L_{2}}}} \chi(x+\xi)\left(-\frac{1}{4} \frac{\partial^{2}}{\partial \xi^{2}}+m^{2}\right)^{\frac{L_{2}+N}{2}} e^{-i \beta \xi \frac{\omega_{k}^{N}}{\omega_{\beta / 2}^{N}}} \\
\times\left(1-\frac{\alpha^{2}}{4} \frac{\partial^{2}}{\partial k^{2}}\right)^{L_{1}} C\left(x ; k-\frac{\alpha \beta}{2}\right) .
\end{aligned}
$$


Choose $L_{2}$ to be such a number that $\frac{L_{2}+N}{2}$ is integer, $L_{2}>d$. The property $\chi \in \mathcal{C}$ implies that there exists such $K$ that

$$
\frac{\partial^{m}}{\partial \xi_{i_{1} \ldots \partial \xi_{i_{n}}}} \chi(x+\xi)=\left((x+\xi)^{2}+1\right)^{K} f_{m . i_{1} \ldots i_{m}}(x+\xi), \quad m=\overline{0, \frac{L_{2}+N}{2}}
$$

where $f_{m, i_{1} \ldots i_{m}}$ are bounded functions. Choose $L_{1}$ to be integer and $L_{1}>\frac{K+d}{2}$. Integrating expression (A.11) by parts, making use of corollary of lemma A.10 and property $C \in \mathcal{A}_{N}$, we obtain that $\omega_{k}^{N} F$ is a bounded function. Lemma A.11 is proved.

Lemma A.12. Under conditions of lemma $A .11 F \in \mathcal{A}_{N}$.

Proof. It is sufficient to consider the functions

$$
\omega_{k}^{N+I} \frac{\partial^{I}}{\partial k_{i_{1}} \ldots \partial k_{i_{I}}} x_{j_{1}} \ldots x_{j_{R}} \frac{\partial}{\partial x_{s_{1}}} \ldots \frac{\partial}{\partial x_{s_{P}}} F
$$

which are expressed via linear combinations of integrals of the type (A.9). Lemma A.12 is a corollary of lemma A.11.

Lemma A.13. Let $\mathcal{A}_{N}-\lim _{n \rightarrow \infty} C_{n}=C, \chi \in \mathcal{C}, \varphi \in C[0.1]$. Then $\mathcal{A}_{N}-\lim _{n \rightarrow \infty} F_{n}=F$.

The proof is analogous to lemmas A.11 and A.12.

We obtain therefore the following theorem.

Theorem A.14. 1. Let $f \in \mathcal{C}, B \in \mathcal{A}_{N}$. Then

$$
f * B=f \stackrel{K}{*} B+R_{K}
$$

with $R_{K} \in \mathcal{A}_{N+K+1}$.

2. Let $f \in \mathcal{C}, \mathcal{A}_{N}-\lim _{n \rightarrow \infty} B_{n}=0$. Then $\mathcal{A}_{N+K+1}-\lim _{n \rightarrow \infty}\left(f * B_{n}-f \stackrel{K}{*} B_{n}\right)=0$.

\section{A.3.2 The $x$-independent case}

Let $A=A(k), A \in \mathcal{B}_{M_{1}}, B \in \mathcal{A}_{M_{2}}$. The only nontrivial term is taken to the form:

$$
r_{0 N_{2}}^{(1)}(x, k)=\int_{0}^{1} d \alpha_{2}\left(-\frac{i}{2}\right)^{N_{2}+1} \frac{\left(1-\alpha_{2}\right)^{N_{2}}}{N_{2} !} \int \frac{d \beta_{2} d \xi_{2}}{(2 \pi)^{d}} e^{-i \beta_{2} \xi_{2}} \frac{\partial^{N_{2}+1} A\left(k+\frac{\alpha_{2} \beta_{2}}{2}\right)}{\partial k^{i_{1}} \ldots \partial k^{i_{2}+1}} \frac{\partial^{N_{2}+1} B\left(x+\xi_{2} ; k\right)}{\partial x^{i_{1}} \ldots \partial x^{i_{2}+1}} .
$$

Lemma A.15. $C=C(k), C \in \mathcal{B}_{K_{1}}, K_{1}>0, D \in \mathcal{A}_{K_{2}}, \varphi \in C[0,1]$. Then for

$$
F(x, k)=\int_{0}^{1} d \alpha \varphi(\alpha) \int \frac{d \beta d \xi}{(2 \pi)^{d}} e^{-i \beta \xi} C\left(k+\frac{\alpha \beta}{2}\right) D(x+\xi, k) \xi_{j_{1}} \ldots \xi_{j_{m}}
$$

the function $\omega_{k}^{K_{1}+K_{2}} F$ is bounded.

Proof. Inserting the identity (A.10) and integrating by parts, we obtain that

$$
\begin{gathered}
F(x, k)=\int_{0}^{1} d \alpha \varphi(\alpha) \int \frac{d \beta d \xi}{(2 \pi)^{d}} \frac{1}{\left(\xi^{2}+1\right)^{L_{1}}} e^{-i \beta \xi} D(x+\xi, k)\left(1-\frac{\alpha^{2}}{4} \frac{\partial^{2}}{\partial k^{2}}\right)^{L_{1}} \\
\times\left(-\frac{i \alpha}{2}\right)^{m} \frac{\partial}{\partial k_{j_{1}}} \ldots \frac{\partial}{\partial k_{j_{m}}} C\left(k+\frac{\alpha \beta}{2}\right) .
\end{gathered}
$$

For the function $\omega_{k}^{K_{1}+K_{2}} F$, one has

$$
\begin{aligned}
\omega_{k}^{K_{1}+K_{2}} F(x, k) & =\int_{0}^{1} d \alpha \varphi(\alpha) \int \frac{d \beta d \xi}{(2 \pi)^{d}} \frac{1}{\left(\xi^{2}+1\right)^{L_{1}} \omega_{\beta / 2}^{L_{2}}} \omega_{k}^{K_{2}} D(x+\xi, k)\left(-\frac{1}{4} \frac{\partial^{2}}{\partial \xi^{2}}+m^{2}\right)^{\frac{L_{2}+K_{1}}{2}} \\
& e^{-i \beta \xi} \frac{\omega_{k}^{K_{1}}}{\omega_{\beta / 2}^{K_{1}}}\left(1-\frac{\alpha^{2}}{4} \frac{\partial^{2}}{\partial k^{2}}\right)^{L_{1}}\left(-i \frac{\alpha}{2}\right)^{m} \frac{\partial^{m}}{\partial k_{j_{1}} \ldots \partial k_{j_{m}}} C\left(k+\frac{\alpha \beta}{2}\right)
\end{aligned}
$$

Integrating by parts for sufficiently large $L_{1}, L_{2}$, making use of lemmas A.10, we check proposition of lemma A.15. 
Lemma A.16. Under conditions of lemma $A .15 F \in \mathcal{A}_{K_{1}+K_{2}}$.

Lemma A.17. Let $\mathcal{A}_{K_{2}}-\lim _{n \rightarrow \infty} D_{n}=D, C=C(k), C \in \mathcal{B}_{K_{1}}, K_{1}>0, \varphi \in C[0,1]$. Then $\mathcal{A}_{K_{1}+K_{2}}-\lim _{n \rightarrow \infty} F_{n}=F$.

The proof is analogous to lemmas A.12 and A.13. We obtain then the following theorem.

Theorem A.18. 1. Let $A=A(k), A \in \mathcal{B}_{M_{1}}, B \in \mathcal{A}_{M_{2}}$. Then

$$
A * B=A \stackrel{K}{*} B+R_{K}
$$

with $R_{K} \in \mathcal{A}_{M_{1}+M_{2}+K+1}$, provided that $K+M_{1}+1>0$.

2. Let $A=A(k), A \in \mathcal{B}_{M_{1}}, \mathcal{A}_{M_{2}}-\lim _{n \rightarrow \infty} B_{n}=B$. Then

$$
\mathcal{A}_{M_{1}+M_{2}+K+1}-\lim _{n \rightarrow \infty}\left(A * B_{n}-A \stackrel{K}{*} B_{n}\right)=0
$$

provided that $K+M_{1}+1>0$.

Remark. If the proposition of theorem A.18 is satisfied for $K=K_{0}$, it is satisfied for all $K \leq K_{0}$. Therefore, the condition $K+M_{1}+1>0$ can be omitted.

The following lemma is a corollary of theorem A.18.

Lemma A.19.1. Let $A \in \mathcal{A}_{N}, N>d$. Then $\mathcal{W}(A)$ is of the trace class.

2. Let $\mathcal{A}_{N}-\lim _{n \rightarrow \infty} A_{n}=0, N>d$. Then $\lim _{n \rightarrow \infty} \operatorname{Tr} \mathcal{W}\left(A_{n}\right)=0$.

Proof. Consider the operator

$$
\hat{B}=\mathcal{W}(B)=\hat{\omega}^{N / 2}\left(x^{2}+1\right)^{N / 2} \mathcal{W}(A)
$$

with

$$
B=\omega_{k}^{N / 2} *\left(x^{2}+1\right)^{N / 2} * A
$$

Since $B \in \mathcal{A}_{N / 2}, \mathcal{W}(B)$ is a Hilbert-Schmidt operator according to lemma A.9. Therefore, $\mathcal{W}(A)$ is a product of two Hilbert-Schmidt operators $\left(x^{2}+1\right)^{-N / 2} \hat{\omega}^{-N / 2}$ and $\mathcal{W}(B)$. Thus, $\mathcal{W}(A)$ is of the trace class.

One also has:

$$
\left|\operatorname{Tr} \mathcal{W}\left(A_{n}\right)\right|=\left|\operatorname{Tr}\left(x^{2}+1\right)^{-N / 2} \hat{\omega}^{-N / 2} \mathcal{W}\left(B_{n}\right)\right| \leq\left\|\left(x^{2}+1\right)^{-N / 2} \hat{\omega}^{-N / 2}\left|{ }_{2}\right| \mid \mathcal{W}\left(B_{n}\right)\right\|_{2}
$$

Making use of lemma A.9, we prove lemma A.19.

\section{A.3.3 The $\mathcal{A}_{N}$-case}

Let $A \in \mathcal{A}_{M_{1}}, B \in \mathcal{A}_{M_{2}}$. The $r$-terms can be investigated as follows.

1. We substitute $\beta_{1,2}^{j} e^{-i \beta_{1,2} \xi_{1.2}} \equiv i \frac{\partial}{\partial \xi_{1,2}^{j}} e^{-i \beta_{1,2} \xi_{1.2}}$ and integrate the expressions for $r^{(1)}, r^{(2)}, R$ by parts with respect to $\xi_{1}, \xi_{2}$.

2. We consider the quantities like

$$
\omega_{k}^{N_{1}+N_{2}+M_{1}+M_{2}+1+L} \frac{\partial^{L}}{\partial k^{i_{1}} \ldots \partial k^{i_{L}}} x_{j_{1}} \ldots x_{j_{J}} \frac{\partial}{\partial x_{s_{1}}} \ldots \frac{\partial}{\partial x_{s_{P}}} r
$$

for $r=r^{(1)}, r^{(2)}, R$ and show them to be bounded. We use the following statement.

Lemma A.20. Let $F \in \mathcal{A}_{K_{1}}, G \in \mathcal{A}_{K_{2}}, K_{1}, K_{2}>0$. Then the function

$$
\int \frac{d \beta_{1} d \beta_{2} d \xi_{1} d \xi_{2}}{(2 \pi)^{2 d}} e^{-i \beta_{1} \xi_{1}-i \beta_{2} \xi_{2}} \omega_{k}^{K_{1}+K_{2}} F\left(x+\xi_{1}, k+\alpha_{2} \frac{\beta_{2}}{2}\right) G\left(x+\xi_{2}, k-\alpha_{1} \frac{\beta_{1}}{2}\right) \xi_{1}^{j_{1}} \ldots \xi_{1}^{j_{m}}
$$

is uniformly bounded with respect to $\alpha_{1}, \alpha_{2} \in[0,1]$.

This lemma is proved analogously to lemmas A.11 and A.15.

3. Analogously to previous subsubsections, we prove the following theorem. 
Theorem A.21. 1. Let $A \in \mathcal{A}_{M_{1}}, B \in \mathcal{A}_{M_{2}}$. Then

$$
A * B=A \stackrel{K}{*} B+R_{K}
$$

with $R_{K} \in \mathcal{A}_{M_{1}+M_{2}+K+1}$.

2. Let $A_{n} \in \mathcal{A}_{M_{1}}, B_{n} \in \mathcal{A}_{M_{2}}$. Then

$$
\mathcal{A}_{M_{1}+M_{2}+K+1}-\lim _{n \rightarrow \infty}\left(A_{n} * B_{n}-A_{n} \stackrel{K}{*} B_{n}\right)=A * B-A \stackrel{K}{*} B .
$$

\section{A.4 Properties of the exponent}

Let us investigate now the properties of the exponent of the operator $\exp \mathcal{W}(A) \equiv \mathcal{W}(* \exp A)$. It is convenient to consider the Fourier transformations of Weyl symbols,

$$
\tilde{A}(\gamma, k)=\int \frac{d x}{(2 \pi)^{d}} e^{-i \gamma x} A(x, k) .
$$

Introduce the following norms for Weyl symbols,

$$
\|A\|_{I, K}=\max _{J+M+N \leq K} \max _{\gamma, K}\left|\omega_{k}^{I+J} \frac{\partial^{J}}{\partial k_{j_{1}} \ldots \partial k_{j_{J}}} \gamma_{m_{1}} \ldots \gamma_{m_{M}} \frac{\partial^{N} \tilde{A}}{\partial \gamma_{n_{1}} \ldots \partial \gamma_{n_{N}}}\right| .
$$

Lemma A.22. $A \in \mathcal{A}_{I}$ if and only if $\|A\|_{I, K}<\infty$ for all $k=\overline{0, \infty}$.

The proof is obvious.

Let $C=A * B$. Then the Fourier transformation $\tilde{C}$ can be expressed via $\tilde{A}$ and $\tilde{B}$ as follows,

$$
\tilde{C}(\gamma, k)=\int d \alpha \tilde{A}\left(\alpha, k+\frac{\gamma-\alpha}{2}\right) \tilde{B}\left(\gamma-\alpha, k-\frac{\alpha}{2}\right)
$$

The following estimation is satisfied.

Lemma A.23. For arbitrary integer numbers $K, L>d / 2$ there exists such a constant $b_{K}$ that

$$
\|A * B\|_{0, K} \leq b_{K}\|A\|_{0, K+2 L}\|B\|_{0, K}
$$

To prove estimation (A.15), one should use definition (A.13) and formula (A.14):

(i) the derivatives $\partial / \partial \gamma_{n}$ are applied as

$$
\begin{gathered}
\frac{\partial}{\partial \gamma_{n}}\left(\tilde{A}\left(\alpha, k+\frac{\gamma-\alpha}{2}\right) \tilde{B}\left(\gamma-\alpha, k-\frac{\alpha}{2}\right)\right)=\frac{1}{2} \frac{\partial \tilde{A}}{\partial k_{n}}(\alpha, k+ \\
\left.\frac{\gamma-\alpha}{2}\right) \tilde{B}\left(\gamma-\alpha, k-\frac{\alpha}{2}\right)+\tilde{A}\left(\alpha, k+\frac{\gamma-\alpha}{2}\right) \frac{\partial}{\partial \gamma_{n}} \tilde{B}\left(\gamma-\alpha, k-\frac{\alpha}{2}\right) ;
\end{gathered}
$$

(ii) the derivatives $\partial / \partial k_{j}$ are applied analogously;

(iii) the multiplicators $\gamma_{m}$ are written as $\alpha_{m}+\left(\gamma_{m}-\alpha_{m}\right)$;

(iv) the estimations

$$
\omega_{k} \leq C \omega_{\alpha / 2} \omega_{k-\alpha / 2}, \quad \omega_{k} \leq C \omega_{\frac{\gamma-\alpha}{2}} \omega_{k+\frac{\gamma-\alpha}{2}}
$$

(lemma A.10) are taken into account.

(v) the integrating measure is written as

$$
d \alpha=\frac{d \alpha}{\left(\alpha^{2}+1\right)^{L}}\left(\alpha^{2}+1\right)^{L} .
$$

We obtain the estimation (A.15). 
Consider the Weyl symbol of the exponent

$$
* \exp A t-1=\sum_{n=1}^{\infty} \frac{A^{* n} t^{n}}{n !}
$$

with $A^{* n}=A * \ldots * A$.

Lemma A.24. Let $A \in \mathcal{A}_{M}, M>0$. Then the estimation (A.10) is convergent in the $\|\cdot\|_{0, K}-n o r m$. The estimation $\|* \exp A t-1\|_{0, K} \leq C_{K}$ is satisfied for $t \in[0, T]$.

Proof. One has

$$
\left\|A^{* n}\right\|_{0, K} \leq b_{K}^{n-1}\|A\|_{0, K+2 L}^{n-1}\|A\|_{0, K} \leq b_{K}^{n-1}\|A\|_{0, K+2 L}^{n} .
$$

Therefore,

$$
\|* \exp A t-1\|_{0, K} \leq \sum_{n=1}^{\infty} \frac{1}{b_{K}} \frac{\left(t\|A\|_{0, K+2 L} b_{K}\right)^{n}}{n !} \leq \frac{e^{t\|A\|_{0, K+2 L} b_{K}}-1}{b_{K}} \leq C_{K}
$$

on $t \in[0 . T]$. Lemma A.24 is proved.

Lemma A.25. Let $A \in \mathcal{A}_{M}, M>0$. Then

$$
\sum_{m=N}^{\infty} \frac{A^{* m}}{m !} \in \mathcal{A}_{M N}
$$

Proof. One has

$$
\sum_{m=N}^{\infty} \frac{A^{* m}}{m !}=A^{* N}\left(\frac{1}{N !}+\int_{0}^{1} d \tau \frac{(1-\tau)^{N-1}}{(N-1) !}(* \exp A \tau-1)\right)
$$

Lemma A.24 implies that

$$
\int_{0}^{1} d \tau \frac{(1-\tau)^{N-1}}{(N-1) !}(* \exp A \tau-1) \in \mathcal{A}_{0} .
$$

It follows from theorem A.21 that the symbol (A.17) is of the $\mathcal{A}_{N M^{-}}$-class. Lemma A.25 is proved.

Lemma A.26. Let $A_{n} \in \mathcal{A}_{M}, M>0$ and $\mathcal{A}_{M}-\lim _{n \rightarrow \infty} A_{n}=A$. Then

$$
\mathcal{A}_{M N}-\lim _{n \rightarrow \infty} \sum_{m=N}^{\infty} \frac{A_{n}^{* m}}{m !}=\sum_{m=N}^{\infty} \frac{A^{* m}}{m !} .
$$

Proof. Because relation (A.17) and theorem A.21 it is sufficient to prove that

$$
\mathcal{A}_{0}-\lim _{n \rightarrow \infty} \int_{0}^{1} d t \frac{(1-t)^{N-1}}{(N-1) !}\left(* \exp A_{n} t-* \exp A t\right)=0 .
$$

One has

$$
* \exp A_{n} t-* \exp A t=\int_{0}^{t} d \tau * \exp A(t-\tau) *\left(A_{n}-A\right) * \exp A_{n} \tau .
$$

Making use of lemma A.23, we obtain then estimation (A.18).

\section{A.5 Estimations for the commutator}

Let $\hat{A}=f(\hat{x}), \hat{B}=g(\hat{k})$. To investigate the properties of the commutator $\hat{K}=[\hat{A} ; \hat{B}]$, it is convenient to introduce the notion of $\hat{x} \hat{k}$-symbol of the operator instead of Weyl symbol. For the $\hat{x} \hat{k}$-quantization, the operator $e^{i \beta \hat{x}} e^{i \alpha \hat{k}}$ corresponds to the function $e^{i \beta x} e^{i \alpha k}$. Therefore, the function

$$
A(x, k)=\int d \alpha d \beta \tilde{A}(\alpha, \beta) e^{i \alpha k+i \beta x}
$$


corresponds to the operator

$$
\hat{A}=\int d \alpha d \beta \tilde{A}(\alpha, \beta) e^{i \beta \hat{x}} e^{i \alpha \hat{k}}
$$

For $\hat{x} \hat{k}$-quantization, the ${ }^{*}$-product defined from the relations $\hat{C}=\hat{A} \hat{B}, C=A * B$ has the form [34, 51]

$$
(A * B)(x, k)=\left.A\left(x, k-i \frac{\partial}{\partial y}\right) B(y, k)\right|_{y=x} .
$$

Lemma A.27. 1. Let $A(x, k)=\varphi_{1}(x) \varphi_{2}(k)$ with bounded functions $\varphi_{1}, \varphi_{2}$. Then $\|\hat{A}\|<\infty$.

2. Let $A \in L^{2}\left(\mathbf{R}^{2 d}\right)$. Then $\hat{A}$ is a Hilbert-Schmidt operator.

Proof. 1. One has $\hat{A}=\varphi_{1}(\hat{x}) \varphi_{2}(\hat{k}),\|\hat{A}\| \leq\left\|\varphi_{1}(\hat{x})|||| \varphi_{2}(\hat{k})\right\|=\max \left|\varphi_{1}\right| \max \left|\varphi_{2}\right|<\infty$.

2. One has

$$
\operatorname{Tr} A^{+} A=\frac{1}{(2 \pi)^{2 d}} \int d x d k|A(x, k)|^{2}<\infty .
$$

The commutator $\hat{K}=[f(\hat{x}), g(\hat{k})]$ has the following $\hat{x} \hat{k}$-symbol:

$$
\begin{aligned}
K(x, k)= & {\left[g(k)-g\left(k-i \frac{\partial}{\partial x}\right)\right] f(x)=\sum_{n=0}^{L} \frac{\partial^{n} g}{\partial k^{i} \ldots \partial k^{i_{n}}}(-i)^{n} \frac{\partial^{n} f}{\partial x^{i} \ldots \partial x^{i_{n}}} } \\
& -\int_{0}^{1} d \alpha \frac{(1-\alpha)^{L}}{L !}(-i)^{L+1} \frac{\partial^{L+1} g\left(k-i \alpha \frac{\partial}{\partial x}\right)}{\partial k^{i_{1} \ldots \partial k^{i} L+1}} \frac{\partial^{L+1} f}{\partial x^{i_{1} \ldots \partial x^{i} L+1}} .
\end{aligned}
$$

Lemma A.28. Let $C(x, k)=A(k-i \alpha \partial / \partial x) B(x)$. Then $\|C\|_{L^{2}}=\|A\|_{L^{2}}\|B\|_{L^{2}}$.

Proof. Consider the Fourier transformation of the function $A$ :

$$
A(k)=\int d \gamma \tilde{A}(\gamma) e^{i \gamma k}
$$

One has $\|A\|_{L^{2}}=(2 \pi)^{d / 2}\|\tilde{A}\|_{L^{2}}$ and

$$
C(x, k)=\int d \gamma \tilde{A}(\gamma) e^{i \gamma k} e^{\gamma \alpha \frac{\partial}{\partial x}} B(x)
$$

Since $e^{\gamma \alpha \frac{\partial}{\partial x}} B(x)=B(x+\gamma \alpha)$, one has

$$
\begin{gathered}
\|C\|_{L^{2}}^{2}=\int d k d x d \gamma_{1} d \gamma_{2} \tilde{A}^{*}\left(\gamma_{1}\right) e^{-i \gamma_{1} k} B^{*}\left(x+\gamma_{1} \alpha\right) \tilde{A}\left(\gamma_{2}\right) e^{i \gamma_{2} k} B\left(x+\gamma_{2} \alpha\right)= \\
(2 \pi)^{d} \int d \gamma|\tilde{A}(\gamma)|^{2} \int d x|B(x+\gamma \alpha)|^{2}=\|A\|_{L^{2}}^{2}\|B\|_{L_{2}}^{2} .
\end{gathered}
$$

Lemma A.28 is proved.

We have obtained the following important statement.

Lemma A.29. Let $\frac{\partial^{n} f}{\partial x^{i_{1}} \ldots \partial x^{i_{n}}}, \frac{\partial^{n} g}{\partial k^{i_{1} \ldots \partial k^{i n}}}$ be bounded functions, $m, n=\overline{1, L}$, while

$$
\frac{\partial^{L+1} f}{\partial x^{i_{1}} \ldots \partial x^{i_{L+1}}} \in L^{2}, \quad \frac{\partial^{L+1} g}{\partial k^{i_{1}} \ldots \partial k^{i_{L+1}}} \in L^{2} .
$$

Then $[f(\hat{x}), g(\hat{k})]$ is a bounded operator.

\section{A.6 Asymptotic expansions of Weyl symbol}

To check the property of Poincare invariance, it is important to investigate the large- $k$ expansion of the Weyl symbols. Introduce the correponding definitions.

Definition A.4. 1. We say that a smooth function $A(x, n), x, n \in \mathbf{R}^{d},|n|<1$, is of the calss $\mathcal{L}$ if the functions

$$
\frac{\partial^{I}}{\partial n_{i_{1}} \ldots \partial n_{i_{I}}} x_{j_{1}} \ldots x_{j_{J}} \frac{\partial^{M}}{\partial x_{m_{1}} \ldots \partial x_{m_{M}}} A
$$


are bounded.

2. Let $A_{s} \in \mathcal{L}, s=\overline{1, \infty}$. We say that $\mathcal{L}-\lim _{s \rightarrow \infty} A_{s}=0$ if

$$
\sup _{|n| \leq 1} \lim _{s \rightarrow \infty}\left|\frac{\partial^{I}}{\partial n_{i_{1}} \ldots \partial n_{i_{I}}} x_{j_{1}} \ldots x_{j_{J}} \frac{\partial^{M}}{\partial x_{m_{1}} \ldots \partial x_{m_{M}}} A\right|=0 .
$$

Definitions A.2 and A.4 imply the following statement.

Lemma A.30. 1. Let $A \in \mathcal{L}$. Then the function $B(x, k)=A\left(x, k / \omega_{k}\right)$ is of the class $\mathcal{B}_{0}$.

2. Let $\mathcal{L}-\lim _{s \rightarrow \infty} A_{s}=0$. Then $\mathcal{B}_{0}-\lim _{s \rightarrow \infty} A_{s}\left(x, k /\right.$ omega $\left.a_{k}\right)=0$.

Making use of definition A.2 and lemma A.25, we obtain the following corollary.

Corollary. 1. Let $A \in \mathcal{L}$. Then the function $\omega_{k}^{-\alpha} A\left(x, k / \omega_{k}\right)$ is of the class $\mathcal{A}_{\alpha}$.

2. Let $\mathcal{L}-\lim _{s \rightarrow \infty} A_{s}=0$. Then $\mathcal{A}_{\alpha}-\lim _{s \rightarrow \infty} \omega_{k}^{-\alpha} A_{s}\left(x, k /\right.$ omega $\left.a_{k}\right)=0$.

Definition A.5. 1. A formal asymptotic expansion is a set $\check{A}$ of $\alpha \in \mathbf{R}$ and functions $A_{0}, A_{1}, \ldots \in \mathcal{L}$. We say that the formal asymptotic expansions $\check{A}=\left(\alpha, A_{0}, A_{1}, ..\right)$ and $\check{B}=\left(\beta, B_{0}, B_{1}, ..\right)$ are equivalent if $\alpha-\beta$ is an integer number and $A_{l-\alpha+\beta}=B_{l}$ for all $l=\overline{-\infty,+\infty}$ (we assume $A_{l}=0$ and $B_{l}=0$ for $l<0$. We denote formal asymptotic expansions of Weyl symbols as

$$
\check{A} \equiv \sum_{n=0}^{\infty} \omega_{k}^{-n-\alpha} A_{n}\left(x, k / \omega_{k}\right) .
$$

If $A_{0}=0, \ldots, A_{l-1}=0, A_{l} \neq 0$, the quantity deg $\check{A} \equiv \alpha+n$ is called as a degree of a formal asymptotic expansion $\check{A}$.

2. Let $\check{A}_{s}, s=\overline{1, \infty}$ and $\check{A}$ be formal asymptotic expansions of Weyl symbols. We say that F.E $\lim _{s \rightarrow \infty} \check{A}_{s}=A$ if $\alpha_{s}=\alpha$ and $\mathcal{L}-\lim _{s \rightarrow \infty}\left(A_{s, n}-A_{s}\right)=0$.

The summation and multiplication by numbers are obviously defined:

$$
\check{A}+\lambda \check{B}=\sum_{n=0}^{\infty} \omega_{k}^{-n-\alpha}\left(A_{n}\left(x, k \omega_{k}\right)+\lambda B_{n}\left(x, k / \omega_{k}\right)\right) .
$$

The product of formal asymptotic expansions of Weyl symbols

$$
\check{A} \equiv \sum_{n=0}^{\infty} \omega_{k}^{-n-\alpha} A_{n}\left(x, k / \omega_{k}\right), \quad \check{B} \equiv \sum_{n=0}^{\infty} \omega_{k}^{-n-\beta} B_{n}\left(x, k / \omega_{k}\right)
$$

is defined as

$$
\check{A} \check{B} \equiv \sum_{n=0}^{\infty} \omega_{k}^{-n-\alpha-\beta} \sum_{s, l \geq 0 ; s+l=n} A_{s}\left(x, k / \omega_{k}\right) B_{l}\left(x, k / \omega_{k}\right) .
$$

Let $f=f(x), f \in \mathcal{C}$. Then

$$
f(x) \check{A} \equiv \sum_{n=0}^{\infty} \omega_{k}^{-n-\alpha} f(x) A_{n}\left(x, k / \omega_{k}\right) .
$$

One also defines

$$
\omega_{k}^{-\beta} \check{A} \equiv \sum_{n=0}^{\infty} \omega_{k}^{-n-\alpha-\beta} A_{n}\left(x, k / \omega_{k}\right)
$$

and

$$
\frac{\partial \check{A}}{\partial k_{s}}=\left.\sum_{l=0}^{\infty} \omega_{k}^{-l-\alpha-1}\left[-(l+\alpha) A_{l}(x, n)+\frac{\partial A_{l}}{\partial n_{p}}(x, n)\left(\delta_{p s}-n_{p} n_{s}\right)\right]\right|_{n=k / \omega_{k}}
$$

The *-product of formal asymptotic expansions is introduced as

$$
\begin{gathered}
\check{A} * \check{B} \equiv \sum_{K=0}^{\infty} \sum_{n_{1} n_{2} \geq 0, n_{1}+n_{2}=K} \frac{i^{n_{1}-n_{2}}}{n_{1} ! n_{2} ! 2^{n_{1}+n_{2}}} \frac{\partial^{n_{1}+n_{2}}}{\partial x^{i_{1}} \ldots \partial x^{i n_{2}} \partial k^{j_{1}} \ldots \partial k^{j_{1}}{ }} \sum_{l_{1}=0}^{\infty} \omega_{k}^{-l_{1}-\alpha_{1}} A_{l_{1}}\left(x, k / \omega_{k}\right) \\
\times \frac{\partial^{n_{1}+n_{2}}}{\partial x^{j_{1}} \ldots \partial x^{j_{1}} \partial k^{i_{1}} \ldots \partial k^{i_{2}}} \sum_{l_{2}=0}^{\infty} \omega_{k}^{-l_{2}-\alpha_{2}} A_{l_{2}}\left(x, k / \omega_{k}\right)
\end{gathered}
$$


The formal asymptotic expansions $\check{A} * \omega_{k}^{\alpha}, \check{A} * f(x)$ are defined analogously. The *-exponent of a formal asymptotic expansion $\check{A}$ is defined as

$$
* \exp \check{A}-1=\sum_{n=1}^{\infty} \frac{\check{A}^{* n}}{n !}
$$

provided that $\operatorname{deg} A$ is a positive integer number.

Definition A.6. 1. An asymptotic expansion of the Weyl symbol is a set $\underline{A} \equiv(A, \check{A})$ of the Weyl symbol $A$ and a formal asymptotic expansion $\check{A}$ such that

$$
A(x, k)-\sum_{l=0}^{n-1} \frac{A_{l}\left(x, k / \omega_{k}\right)}{\omega_{k}^{l+\alpha}} \in \mathcal{A}_{n+\alpha}
$$

for all $n=\overline{0, \infty}$.

2. We say that $E-\lim _{s \rightarrow \infty} \underline{A_{s}}=\underline{A}$ if $F . E-\lim _{s \rightarrow \infty} \check{A}_{s}=\check{A}$ and

$$
\mathcal{A}_{n+\alpha}-\lim _{s \rightarrow \infty}\left(A_{s}(x, k)-\sum_{l=0}^{n-1} \frac{A_{s, l}\left(x, k / \omega_{k}\right)}{\omega_{k}^{l+\alpha}}\right)=A(x, k)-\sum_{l=0}^{n-1} \frac{A_{l}\left(x, k / \omega_{k}\right)}{\omega_{k}^{l+\alpha}}
$$

for all $n=\overline{0, \infty}$.

Remark. For given Weyl symbol $A$, the asymptotic expansion is not unique. For example, let

$$
A(x, k)=m^{2} f(x) / \omega_{k}
$$

One can choose $\alpha=2, A_{0}(x, n)=m^{2} f(x)$ anf find $A(x, k)=\omega_{k}^{-2} A_{0}\left(x, k / \omega_{k}\right)$. On the other hand, one can set $\alpha=0, A_{0}(x, n)=f(x)\left(1-n_{i} n_{i}\right)$ and obtain $A(x, k)=A_{0}\left(x, k / \omega_{k}\right)$ since $\omega_{k}^{2}-k_{i} k_{i}=m^{2}$. We see that a degree is a characteristic feature of an expansion rather than of a symbol.

Let $\underline{A}=(A, \check{A}), \underline{B}=(B, \check{B})$. Denote $\underline{A} * \underline{B} \equiv(A * B, \check{A} * \check{B})$, $\omega_{k}^{\alpha} * \underline{A} \equiv\left(\omega_{k}^{\alpha} * A, \omega_{k}^{\alpha} * \check{A}\right)$, $f(x) * \underline{A} \equiv(f(x) * A, f(x) * \check{A})$, $* \exp \underline{A}-1 \equiv(* \exp A-1, * \exp \check{A}-1)$.

Theorems A.14, A.18, A.21 and lemmas A.25 and A.26 imply the following statements.

Theorem A.31. 1. Let $\underline{A}$ be an asymptotic expansion of a Weyl symbol. Then $\omega_{k}^{\alpha} * \underline{A}$ and $f(x) * \underline{A}$ are asymptotic expansions of Weyl symbols under conditions of theorem A.14, while $* \exp \underline{A}-1$ is an asymptotic expansion of a Weyl symbol, provided that deg $\check{A}$ is a positive integer number.

2. Let $\underline{A}$ and $\underline{B}$ be asymptotic expansions of Weyl symbols. Then $\underline{A} * \underline{B}$ is an asymptotic expansion af a Weyl symbol.

Theorem A.32. 1. Let $E-\lim _{n \rightarrow \infty} \underline{A}_{n}=\underline{A}$. Then:

(a) $E-\lim _{n \rightarrow \infty} \omega_{k}^{\alpha} * \underline{A}_{n} \omega_{k}^{\alpha} * \underline{A}$;

(b) $E-\lim _{n \rightarrow \infty} f(x) * \underline{A}_{n} f(x) * \underline{A}$ under conditions of theorem A.14;

(c) $E-\lim n \rightarrow \infty\left(* \exp \underline{A_{n}}-1\right)=* \exp \underline{A}-1$ if deg $\check{A}_{n}$, deg $\check{A}$ are positive integer numbers.

2. Let $E-\lim _{n \rightarrow \infty} \underline{A}_{n}=\underline{\bar{A}}$ and $E-\lim _{n \rightarrow \infty} \underline{B}_{n}=\underline{B}$. Then $E-\lim _{n \rightarrow \infty} \underline{A}_{n} * \underline{B}_{n}=\underline{A} * \underline{B}$.

The time derivative of teh asymptotic expansion $\underline{A}(t)$ with respect to $t$ is defined in a standard way

$$
E-\lim _{\delta t \rightarrow 0} \frac{\underline{A}(t+\delta t)-\underline{A}(t)}{\delta t}=\frac{d \underline{A}(t)}{d t} .
$$

The integral $\int_{t_{1}}^{t_{2}} \underline{A}(t) d t$ is also defined in a standard way.

Theorem A.32 imply the following statement.

Theorem A.33. 1. Let $\underline{A}(t)$ be a continously differentiable asymptotic expansion of a Weyl symbol. Then

(a) $\frac{d}{d t}\left(\omega_{k}^{\alpha} * \underline{A}\right)=\omega_{k}^{\alpha} * \frac{d \underline{A}}{d t}$;

(b) $\frac{d}{d t}(f(x) * \underline{A})=f(x) * \frac{d \underline{A}}{d t}$ under conditions of theorem A.14. 
(c) $\frac{d}{d t}(* \exp \underline{A}-1)=\int_{0}^{1} d \tau e^{\underline{A}(t-\tau)} * \frac{d \underline{A}}{d t} * e^{\underline{A} \tau}$;

(d) $\frac{d}{d t}(\underline{A} * \underline{B})=\frac{d}{d t} \underline{A} * \underline{B}+\underline{A} * \frac{d}{d t} \underline{B}$.

The only nontrivial statement is (c). It is proved by using the identity [51]

$$
* \exp \underline{A}_{1}-* \exp \underline{A}_{2}=\int_{0}^{1} d \tau * \exp \left(\underline{A}_{1}(1-\tau)\right) *\left(\underline{A}_{1}-\underline{A}_{2}\right) * \exp \left(\underline{A}_{2} \tau\right) \text {. }
$$




\section{References}

[1] R.Dashen, B.Hasslasher and A.Neveu, Phys. Rev. D10 (1974), 4114

[2] R.Rajaraman, "Solitons and Instantons. An Introduction to solitons and instantons in quantum field theory", North-Holland,Amsterdam, Netherlands, 1982.

[3] J. Coldstone and R.Jackiw, Phys.Rev. D11 (1975), 1486

[4] R.Jackiw, Rev.Mod.Phys. 49 (1977), 681.

[5] L.D.Faddeev and V.E.Korepin, Phys. Rep. 42 (1978) 1.

[6] A.A. Grib, S.G. Mamaev and V.M. Mostepanenko, "Vacuum Quantum Effects in Strong Fields", Atomizdat, Moscow, 1988; Friedmann Laboratory Publishing, St. Petersburg 1994.

[7] N.D. Birrell and P.C.W. Davies, "Quantum Fields in Curved Space", Cambridge, UK: Univ. Pr. , 1982.

[8] D.Boyanovsky, H.J. de Vega and R.Holman, Phys. Rev. D49 (1994), 2769.

[9] D.Boyanovsky, H.J. de Vega, R.Holman, D.S.Lee and A.Singh, Phys. Rev. D51 (1995), 4419.

[10] Ju.Baacke, K.Heitmann and C.Pätzold, Phys. Rev. D55 (1997), 2320.

[11] Ju.Baacke, K.Heitmann and C.Pätzold, Phys. Rev. D56 (1997), 6556.

[12] F.Cooper and E.Mottola, Phys.Rev. D36 (1987), 3114

[13] S.-Y.Pi and M.Samiullah, Phys. Rev. D36 (1987), 3128

[14] R.Jackiw and A.Kerman, Phys.Lett. A71 (1979), 158.

[15] F.Cooper, S.-Y.Pi and P.Stancioff, Phys. Rev. D34 (1986), 3831.

[16] O.Eboli, R.Jackiw and S.-Y.Pi, Phys. Rev. D37 (1988), 3557.

[17] O.Eboli, S.-Y.Pi and M.Samiullah, Ann. Phys. 193 (1989), 102.

[18] A.S.Wightman, Phys. Rev. 101 (1956), 860.

[19] R.F.Streater and A.S. Wightman "PCT, Spin and Statistics and All That", N.Y., Benjamin, 1964.

[20] N.N.Bogoliubov, A.A.Logunov, A.I.Oksak and I.T.Todorov, "General Principles of Quantum Field Theory", Moscow, Nauka, 1987; Kluwer Academic Publishers, 1990.

[21] K. Hepp, "Theorie de la renormalisation", Springer-Verlag, 1969.

[22] J. Glimm and A. Jaffe, "Boson quantum field models". In "London 1971, Mathematics Of Contemporary Physics", London 1972, pp. 77-143.

[23] I.Ya.Arefieva, Teor.Mat.Fiz. 14 (1973) 3.

[24] I.Ya.Arefieva, Teor.Mat.Fiz. 15 (1973) 207.

[25] N.N. Bogoliubov and D.V. Shirkov, "Introduction to the Theory of Quantized Fields", N.Y.,Interscience Publishers, 1959. 
[26] A.A.Slavnov and L.D.Faddeev, "Introduction to the quantum theory of gauge fields", Moscow, Nauka, 1988.

[27] E.C.G. Stueckelberg Phys.Rev. 81 (1951) 130.

[28] R.Haag, Kgl. Danske Videnskab. Selsk. Mat.-Fiz. Medd. 29 (1955) N12.

[29] L.D.Faddeev, Doklady Akademii Nauk SSSR 152 (1963) 573.

[30] V.P.Maslov and O.Yu.Shvedov, Trudy MIAN 226 (1999) 112.

[31] O.Yu.Shvedov, Teor. Mat. Fiz. 125 (2000) 91.

[32] O.Yu.Shvedov, hep-th/0002108, Ann. Phys. 287 (2001) 260.

[33] V.P.Maslov and O.Yu.Shvedov Teor. Mat.Fiz. 114 (1988) 233.

[34] V.P.Maslov, "Operational Methods", Moscow, Nauka, 1973; English translation: Moscow, Mir Publishers, 1976.

[35] V.P.Maslov, "The Complex-WKB Method for Nonlinear Equations", Moscow, Nauka, 1977.

[36] V.P.Maslov and O.Yu.Shvedov, "The Complex Germ Method in Many-Particle Problem and Quantum Field Theory", Moscow, Editorial URSS, 2000.

[37] A.A.Grib and S.G.Mamaev, Yadernaya Fizika, 10 (1969), 1276.

[38] M.I.Shirokov, Yadernaya Fizika, 7 (1968) 672.

[39] O.Yu.Shvedov, Matematicheskie zametki 65 (1999) 437

[40] O.Yu.Shvedov, Matematicheskii sborik 190 (1999) N10, 123.

[41] R.Floreanini and R.Jackiw, Phys. Rev. D37 (1988) 2206

[42] F.A.Berezin, "The Method of Second Quantization", Moscow, Nauka, 1965; N.Y.1996.

[43] A.Barut and R.Raczka, "Theory of Group Representations and Applications", Warszawa, Pol.sci.publ., 1977.

[44] E. Nelson, Ann. Math. 70, 572 (1959)

[45] M.Flato, J.Simon, H.Snellman and D.Sternheimer, Ann. Scient. de l'Ecole Norm. Sup. 5, 423 (1972).

[46] J.Simon, Comm. Math. Phys. 28, 39 (1972).

[47] M.Flato and J.Simon, J. Funct. Anal. 13, 268 (1973).

[48] O.Yu.Shvedov, math-ph/0109016

[49] K.Wilson, Phys. Rev. D7 (1974) 2911

[50] J.Collins, "Renormalization. An Introduction to Renormalization, the Renormalization Group and the Operator-product Expansion". Cambridge, Cambridge University Press, 1984.

[51] M.V.Karasev and V.P.Maslov, "Nonlinear Poisson Bracket. Geometry and Quantization", Moscow, Nauka, 1991. 